\title{
Oscillatory instabilities of intracellular fiber networks
}

\author{
Dissertation \\ for the award of the degree \\ "Doctor rerum naturalium" \\ of the Georg-August-Universität Göttingen \\ within the doctoral program \\ IMPRS Physics of Biological and Complex Systems \\ of the Georg-August University School of Science (GAUSS)
}

\author{
submitted by \\ Hsin-Fang Hsu \\ from Taiwan
}

Göttingen, 2015 


\section{Thesis Committee:}

\section{Prof. Dr. Eberhard Bodenschatz}

Department of Fluid Dynamics, Pattern Formation and Biocomplexity

Max Planck Institute for Dynamics and Self-Organization

Institute for Nonlinear Dynamics

Georg-August-Universität Göttingen

Prof. Dr. Jörg Enderlein

III. Physical Institute Biophysics / Complex Systems

Georg-August-Universität Göttingen

Prof. Dr. Andreas Janshoff

Institute for Physical Chemistry

Georg-August-Universität Göttingen

\section{Members of Examination Board:}

Prof. Dr. Thomas Burg

Biological Micro- and Nanotechnology

Max Planck Institute for Biophysical Chemistry

Dr. Marco Tarantola

Department of Fluid Dynamics, Pattern Formation and Biocomplexity

Max Planck Institute for Dynamics and Self-Organization

Prof. Dr. Jörg Großhans

Department Developmental Biochemistry

Institute of Biochemistry and Molecular Cell Biology

University Medical Center Göttingen

Date of oral examination: 2015/05/19 

1 Introduction 9

1.1 Dictyostelium discoideum . . . . . . . . . . . . . . . . . . . 9 9

1.2 Actin cytoskeleton . . . . . . . . . . . . . . . . . . 14

1.3 Myosin II . . . . . . . . . . . . . . . . . . . . . . . . 20

1.4 Self-organization and oscillations . . . . . . . . . . . . . 26

1.5 Aim and outline . . . . . . . . . . . . . . . 27

2 Material and methods $\quad 28$

2.1 Cell culture and development . . . . . . . . . . . . . . 28

2.2 Microfluidics . . . . . . . . . . . . . . . . 30

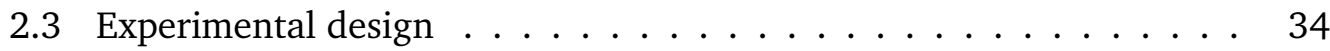

2.4 Image processing and data analysis $\ldots \ldots \ldots . \ldots . \ldots 35$

3 Self-sustained oscillations 43

3.1 Motivation . . . . . . . . . . . . . . . . . 43

3.2 Determining self-oscillations . . . . . . . . . . . . . . 43

3.3 Properties of self-oscillations . . . . . . . . . . . . . . . . . . 47 4

3.4 Impact of various cell properties on self-oscillations . . . . . . . . . 50

3.5 Proposed underlying mechanism . . . . . . . . . . . . . . 52

3.6 Pseudopod formation and self-oscillations . . . . . . . . . . . 54

3.7 Conclusion . . . . . . . . . . . . . . . . . 59 
4 Actin dynamics is stimulation strength dependent 60

4.1 Motivation . . . . . . . . . . . . . . . . 60

4.2 Responses of self-oscillating cells to external stimuli . . . . . . . . . . 62

4.3 Effects of different strengths of stimulation . . . . . . . . . . . . 64

4.4 Single cell experiments . . . . . . . . . . . . . . . . . . . . . . . . . . 67

4.5 Proposed underlying mechanism . . . . . . . . . . . . . . 71

4.6 Nonlinear properties of responses . . . . . . . . . . . . . . 75

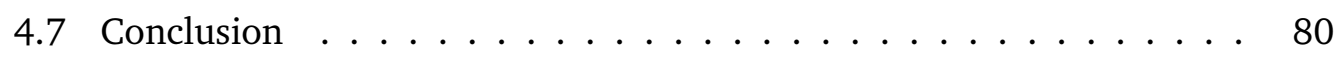

5 Myosin II sets the optimal response time scale 82

5.1 Motivation . . . . . . . . . . . . . . . . . . 82

5.2 Myosin II dynamics in the absence of external stimulation . . . . . . 83

5.3 The dynamics of myosin II in the presence of cAMP stimulation . . 85

5.4 Myosin II helps the recovery of cortical actin filaments. . . . . . . . . 88

5.5 The role of myosin II before its translocation to the cortex . . . . . . 91

5.6 Conclusion .......................... 96

$\begin{array}{lr}\text { Conclusion and Outlook } & 97\end{array}$

$\begin{array}{lr}\text { Bibliography } & 99\end{array}$

$\begin{array}{ll}\text { Acknowledgments } & 113\end{array}$

$\begin{array}{lr}\text { Curriculum Vitae } & 114\end{array}$ 


\section{List of Figures}

1.1 Social cycle of $D$. discoideum. . . . . . . . . . . . . . . 10

1.2 Morphogenesis in the mound. . . . . . . . . . . . . . . . 11

1.3 Comparison of cytoskeleton related proteins in different species. . . . 13

1.4 Examples of actin-based cytoskeleton function. . . . . . . . . . . . . 14

1.5 The structure of an actin monomer. . . . . . . . . . . . . 15

1.6 Formation of actin filaments: nucleation and extension. . . . . . . . 16

1.7 Actin filament elongation and ATP hydrolysis. . . . . . . . . . . . . 17

1.8 Formation of dendritic actin network and the key regulation proteins. 18

1.9 Key actin related proteins in different cells. . . . . . . . . . . . . . . 19

1.10 Schematic representation of the myosins in $D$. discoideum. . . . . . . 21

1.11 The structure of non-muscle myosin II. . . . . . . . . . . . . . . 22

1.12 ATPase cycle of myosin. . . . . . . . . . . . . . . . . . . 23

1.13 Assembly pathways of myosin II filament in D. discoideum. . . . . . . 24

1.14 The structure of myosin II. . . . . . . . . . . . . . . . . . . 25

2.1 The growth of $D$. discoideum cells. . . . . . . . . . . . . . . . . . . . . 29

2.2 Summary of the fabrication of microfluidic channels. . . . . . . . . . 31

2.3 Flow chart of photoresist fabrication. . . . . . . . . . . . . 32

2.4 Structures of caged cAMP. . . . . . . . . . . . . . 35

2.5 A schematic diagram of the experimental setup. . . . . . . . . . . 36

2.6 Method of finding preliminary threshold. . . . . . . . . . . . 37

2.7 Image processing. . . . . . . . . . . . . . . . . 38

2.8 Process to obtain optimal cytosolic signal. . . . . . . . . . . . . . . 40

2.9 Homogeneous cytosolic signal. . . . . . . . . . . . . . . . . . 41

2.10 Local analysis of fluorescence intensity in different regions inside a cell. 42

3.1 Examples of cytosolic intensity in the absence of external stimulation. 44

3.2 Autocorrelation with fixed-size window can reveal the varying properties of the oscilations. . . . . . . . . . . . . . . . 45 
3.3 Self-oscillating cells are synchronized as cells respond to uniform stimulation. . . . . . . . . . . . . . . . 46

3.4 Cytosolic signal and cortical signal oscillate out of phase in cells showing self-oscillation. . . . . . . . . . . . . . . 47

3.5 Distribution of self-oscillation periods. . . . . . . . . . . . . . 48

3.6 To determine self-oscillations by autocorrelation function. . . . . . . 49

3.7 Analysis of oscillation dwell time. . . . . . . . . . . . . . . 50

3.8 Impact of different passage numbers, starvation times and cell sizes in the distribution of self-oscillations. . . . . . . . . . . . . 51

3.9 Capping protein and actin peaked in the cortex at the same time. . . 52

3.10 Learn the time of capping protein binding from the response to cAMP. 53

3.11 Self-oscillating signals show symmetric oscillations. . . . . . . . . . 55

3.12 Arp $2 / 3$ is activated by external stimulation. . . . . . . . . . . 56

3.13 Self-oscillations in the absence of external cAMP was related to the membrane undulation of the cells. . . . . . . . . . . 57

3.14 D. discoideum cells show constant protrusion rate. . . . . . . . . . 58

4.1 Effects of self-oscillations on cAMP response. . . . . . . . . . . 61

4.2 The impact of periodic stimulation on self-oscillation. . . . . . . . . 63

4.3 Response to external stimulation depends on the strength of excitation. 65

4.4 Resonance peak shifts for different stimulation strength. . . . . . . . 66

4.5 A schematic diagram illustrating the experiments that apply different doses of cAMP to single cells. . . . . . . . . . . . . . . . 67

4.6 Threshold for cAMP responses varies a lot among cells. . . . . . . . . 68

4.7 Intrinsic noise causes the scattering of response amplitude. . . . . . . 69

$4.8 \mathrm{~T}_{\mathrm{p}}$ and $\mathrm{T}_{\mathrm{d}}$ at diverse stimulation strength in different cells. . . . . 70

4.9 Global parameters that determine the behavior of cells. . . . . . . . 71

4.10 The dependence of Amp on polymerization and depolymerization. . 72

4.11 The relation between Amp and $T_{d}$ on a log-log scale. . . . . . . . . 73

4.12 Amp- $\mathrm{T}_{\mathrm{d}}$ relation of Aip1-null cells. . . . . . . . . . . . . . . 74

4.13 Simulated resonance curves of damped oscillators. . . . . . . . . . 75

4.14 Effect of altering the strength of stimulation on the resonance curve. 76

4.15 Simulations of resonance curves with different stimulation strengths. $\quad 77$

4.16 Simulation of Amp versus $T_{p}$ and Amp versus $T_{d}$ at different strengths of external stimulation. . . . . . . . . . . . . . 78

4.17 Amount of Aip1 is limited with the increasing of LimE. . . . . . . . 79 
5.1 Oscillations of myosin II and LimE were independent of each other. . 83

5.2 Properties of self-oscillations in myosin II-null cells. . . . . . . . . . 84

5.3 Responses of LimE and myosin II to a single $1 \mathrm{~s}$ pulse of cAMP. . . . . 85

5.4 Schematic diagram showing how myosin II in $D$. discoideum cells changes its localization in response to cAMP. . . . . . . . . . 86

5.5 Periodic stimuli were applied to verify the release of myosin II from the cortical region right after stimulation. . . . . . . . . . 87

5.6 Comparison of LimE dynamics in wild-type and myosin II-null cells. . 88

5.7 Translocation of myosin II to the cortex relies on the dynamics of the actin filaments. . . . . . . . . . . . . . . . . . 89

5.8 Myosin II-null cells cannot contract after stimulation by cAMP. . . . . 90

5.9 Effect of myosin II on filamentous actin dynamics before its translocation to the cortex. . . . . . . . . . . . . . . . . . . 92

5.10 Logarithm fitting of relation between Amp and $\mathrm{T}_{\mathrm{d}} . \quad \ldots \ldots 9$

5.11 Effect of starvation time on actin dynamics in myosin II-null cells. . . 94

5.12 Correlation analysis between cell size and the time cells stay on the solid surface. . . . . . . . . . . . . . . . . . . . . . . 94

5.13 Effect of cell sizes on the actin dynamics in myosin II-null and wild-

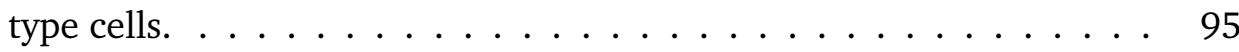




\section{List of Abbreviations}

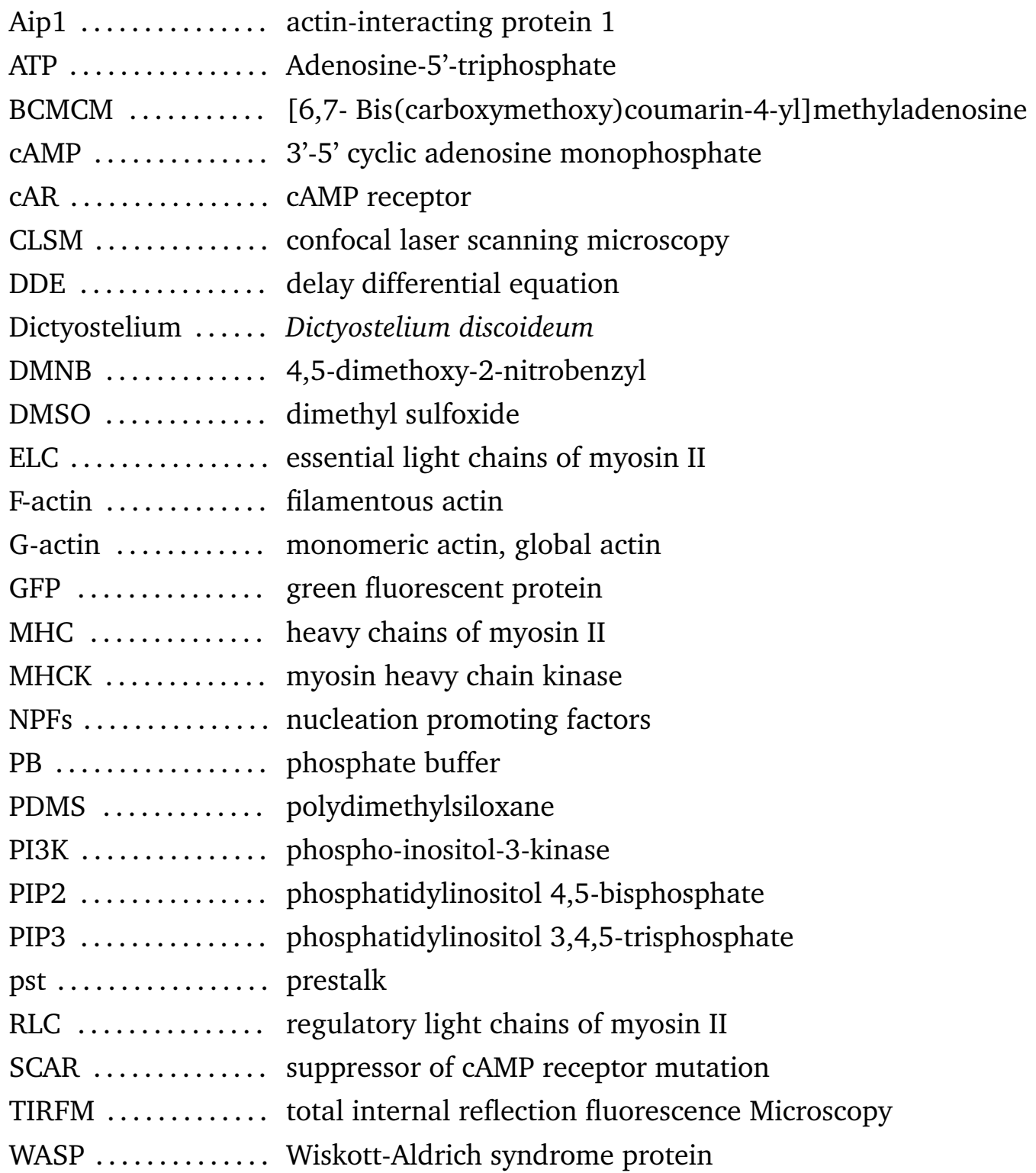




\section{CHAPTER 1}

\section{Introduction}

\subsection{Dictyostelium discoideum}

Dictyostelium discoideum, a slime mold, is well known as being a model system for studying cell differentiation and chemotaxis. D. discoideum was first discovered by Oskar Brefeld in 1869 in horse dung [1]. People grew D. discoideum using dung until 1902, when Potts realized that the growth depends on the presence of bacteria and developed a corresponding culture medium [2]. In 1967, Maurice and Raquel Sussman reported the first cultivation of $D$. discoideum in an axenic culture medium [3], which is a broth medium instead of bacteria to exclude living organisms other than $D$. discoideum from the culture. A modified medium called HL-5 was reported in 1977, which is used until today [4].

Figure 1.1 shows the social cycle of $D$. discoideum. In their vegtative stage, $D$. discoideum cells live as single-celled amoebae in soil. They consume bacteria by phagocytosis (roughly 1,000 bacteria per generation) and multiply by binary fission every 8 hours [6]. Axenic strains can not only perform phagocytosis but also use liquid nutrition by macropinocytosis [7]. Upon the depletion of food, $D$. discoideum cells start to secrete a chemical, 3'-5'-cyclic adenosine monophosphate (cAMP), and develop receptors for cAMP. After sensing cAMP, cells move toward the source of this chemoattractant - a process known as chemotaxis - and also release cAMP to relay the signal. The first group of cells producing cAMP then becomes the aggregation center, while cAMP waves propagate outward from there. Cells cringe (i.e. become roundish) as the cAMP waves pass by; the shape change induced by cAMP is reflected in the intensity change of the scattered light, ultimately leading to excitation patterns resembling spiral waves [8-13]. On the other hand, the chemotatic response also leads the cells to move toward the aggregation center. The so called streaming of 


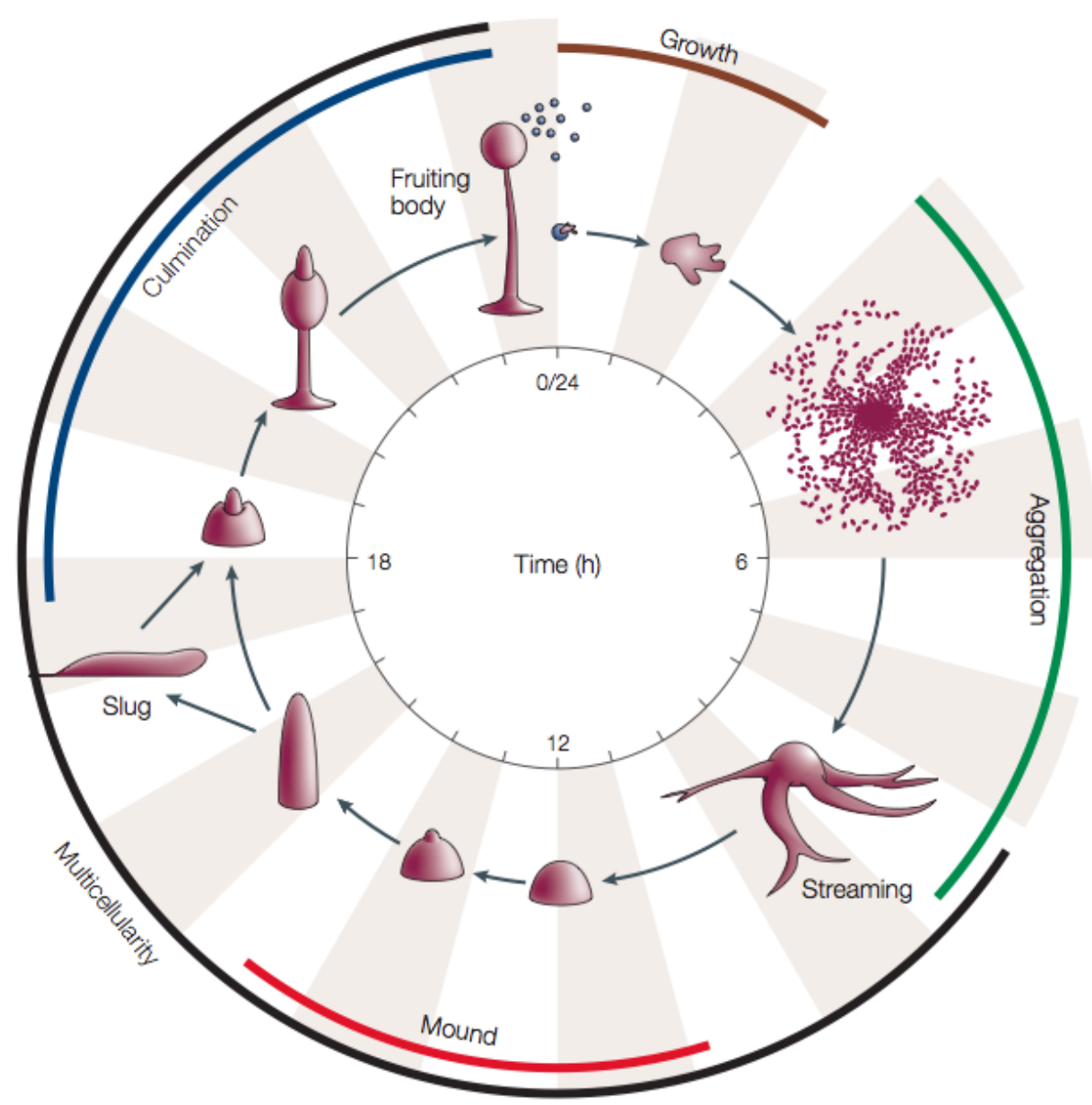

Figure 1.1: Social cycle of $D$. discoideum. Spores can germinate when the environment is suitable for growth. However, upon deprivation of food, cells start to move toward each other via chemotaxis. They aggregate to become multicellular, starting from a mound and then differentiate through a slug into a fruiting body. Adapted by permission from Macmillan Publishers Ltd: Nature Reviews Molecular Cell Biology [5], copyright (2004). 
cells is represented by a series of radial arms and ends up with the formation of a multicellular organism. The structure is known as a mound and is composed of up to $10^{5}$ cells. Cells change their behaviors in the mound according to their starvation history: cells starved in the S and the early G2 phase differentiate into prestalk cells, whereas cells starved in the late G2 phase become prespore cells[5, 14-16].

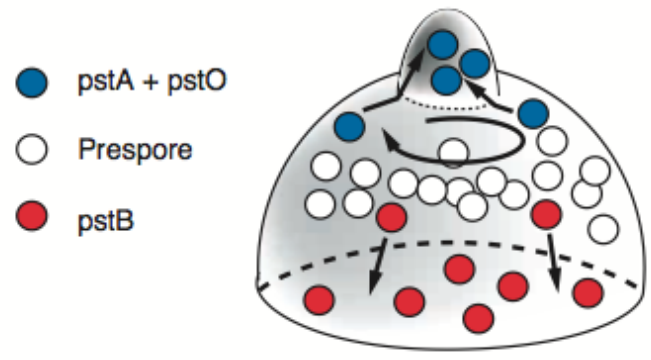

Figure 1.2: Morphogenesis in the mound. To form the stalk of a fruiting body, prestalk cells pstA and pstO move upwards to the tip and pstB cells move downwards to the bottom. Republished with permission of Company of Biologists Ltd., from [17]; permission conveyed through Copyright Clearance Center, Inc.

It is the different chemotatic responses between prestalk and prespore cells that mediates the differentiation: prestalks cells move faster so the prestalk $\mathrm{A}$ and the prestalk O (pstA and pstO) cells rotate toward the tip and the prestalk B (pstB) cells move to the base, while the slower moving prespore cells stay in the central mound (Figure 1.2). The tip can further elongate to form a finger-like structure, which becomes the slug. The slug can migrate to find the ideal environmental conditions, failing which it transforms into a fruiting body. During the process of fruiting body formation - known as culmination - prestalk cells differentiate into the stalk and the basal disk and raise the prespore cells to the top. The stalk cells die afterwards but the remaining spore cells are stored in the spherical structure called sorus and can be dispersed as spores. The name $D$. discoideum actually reflects the observation of fruiting body production. Dicty means "net-like" structure of many aggregating cells while -stelium means "tower", representing the standing stalk [1]. D. discoideum can also incorporate bacteria into their fruiting bodies so that they can be carried away as new food source during dispersion of the spores [18]. Spores are resistant to heating, freezing or drying. Once the environment is suitable for growing, the spores will germinate, $D$. discoideum cells emerge and the life cycle starts again $[19,20]$. It is the ability to switch between the single and the multicellular forms that justifies calling D. discoideum in this part of its life cycle, "social" [21]. 
D. discoideum cells are easy to culture and genetically modify [21, 22]. Unlike mammalian cells, $D$. discoideum cells grow at room temperature and under atmospheric $\mathrm{CO}_{2}$ levels. Moreover, $D$. discoideum is haploid (i.e., $D$. discoideum has only one set of chromosomes). So genes need to be knocked out just once to create mutants. This property is very helpful in the creation of mutations. Protocols for genetic modifications with methods like targeted gene disruption [23], restriction enzyme mediated integration (REMI) [24] or RNA interference inhibition [25] have been developed over the years. Furthermore, the recently sequenced $D$. discoideum genome supports genetic modifications and the evolutional comparison to other species [26].

Besides the experimental advantages, the genome sequence shows that despite the fact that $D$. discoideum diverged from metazoa earlier than fungus ( $D$. discoideum diverges right after the split of animal-plant), D. discoideum preserved more cytoskeleton related proteins that are similar to metazoa than to fungus and plants (Figure 1.3) [26]. The cytoskeleton of eukaryotic cells is essential to maintain the integrity of cells as well as to resist forces. It also plays important roles in selforganization for migration, division during cytokinesis as well as transport of vesicles inside cells. Cytoskeleton also drives ubiquitous and significant reactions such as phargocytosis and chemotaxis. Also, the morphogenesis of D. discoideum cells is triggered by physiological stimuli, which enables the direct study of chemotaxis. Taken together, $D$. discoideum is a well established model system for the study of eukaryotic cytoskeleton and chemotaxis. 


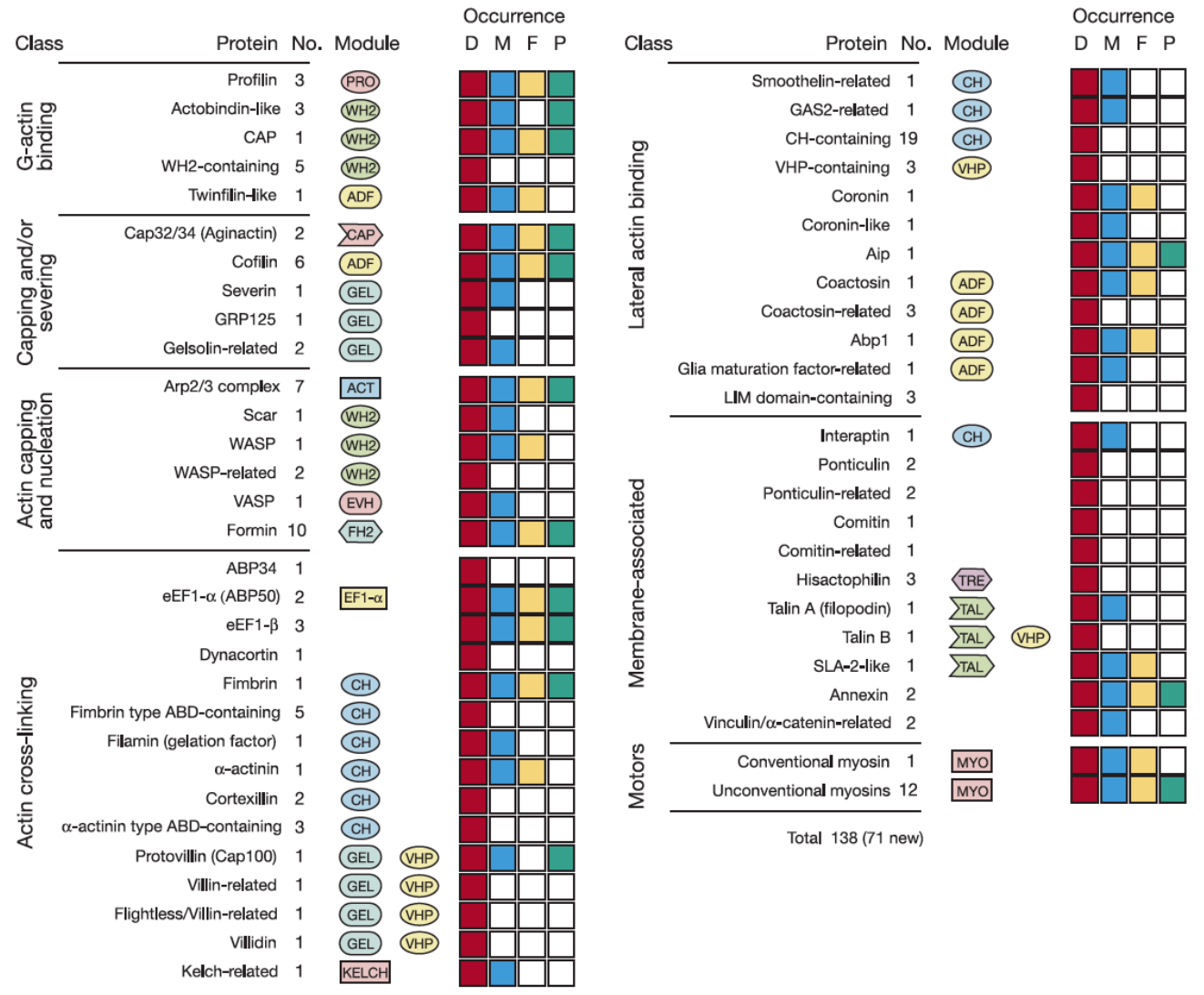

Figure 1.3: Comparison of cytoskeleton related proteins in D. discoideum (D), Metazoa (M), fungi (F) and plants (P). ADF, actin depolymerization factor/cofilin-like domain; CAP, capping protein fold; $\mathrm{CH}$, calponin homology domain; EVH, Ena/VASP homology domain 2; FH2, formin homology 2 domain; GEL, gelsolin repeat domain; KELCH, Kelch repeat domain; MYO, myosin motor domain; PRO, profilin fold; TAL, the I/LWEQ actin-binding domain of talin and related proteins; TRE, trefoil domain; VHP, villin head piece; WH2, Wiskott Aldrich syndrome homology region 2. Adapted by permission from Macmillan Publishers Ltd on behalf of Cancer Research UK: Nature [26], copyright (2005). 


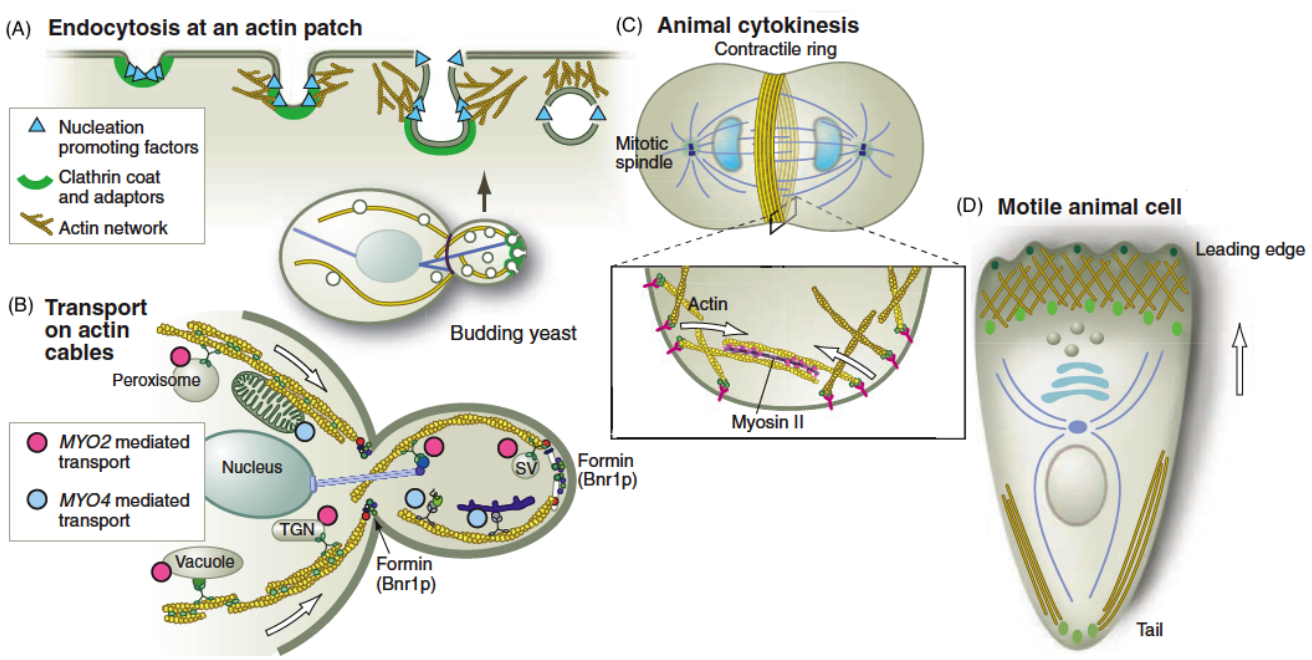

Figure 1.4: Examples of actin-based cytoskeleton function. (A) The role of actin in endocytosis. (B) Actin filaments act as tracks for myosin motors to transport organelles. (C) Actin and myosin motors form a contractile ring to produce traction force in the end of cytokinesis. (D) As a part of the cytoskeleton, actin not only supports the structure of the cell but also protrudes the membrane to enable movement of the cell. Figure was modified from [27].

\subsection{Actin cytoskeleton}

The cytoskeleton is a fiber network composed of various proteins inside the cellular protein matrix of the cytoplasm. It is of extreme importance to maintain the cell structure. Moreover, its dynamic function enables cells to respond instantly to environmental changes. The cytoskeleton is found in all cells, from bacteria to mammalian cells with very similar preserved building components.

The cytoskeleton of eukaryotic cells is composed of three types of filaments: actin microfilaments, intermediate filaments, and microtubules. Microfilaments are composed of actin and are the thinnest of the cytoskeletal filaments (around $7 \mathrm{~nm}$ ). The dynamic behavior of microfilaments plays an important role not only in maintaing integrity and stiffness of cells, but also in determining cell devision, development as well as movement. The present work is focused on the dynamics of microfilaments. Intermediate filaments have, according to their name an intermediate thickness amnog the filaments of cytoskeleton (with diameter around $10 \mathrm{~nm}$ ). Their main function is to maintain the cell shape but they are not directly involved in cell moltility. However, D. discoideum does not have intermediate filaments. A microtubule is composed of $\alpha-$ and $\beta$ - tubulin. The diameter is around $23 \mathrm{~nm}$. Microtubules are the most rigid cytoskeletal filaments. They serve as tracks for motor 
proteins such as kinesins and dyneins which transport organelles inside the cells. Microtubules also form the mitotic spindle and thus are of great importance in cell division.

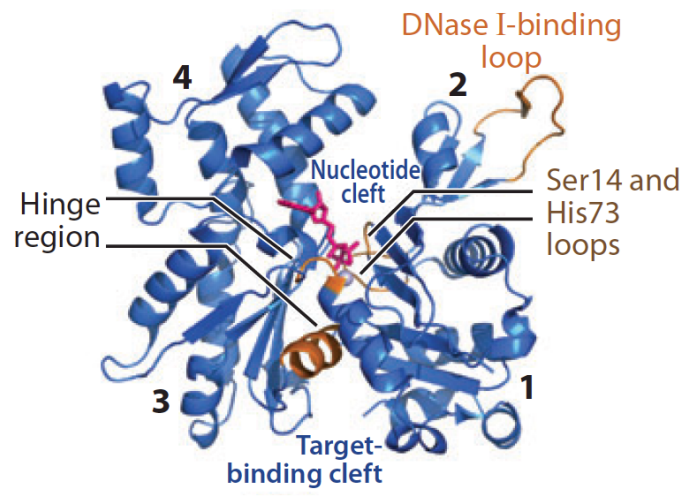

Figure 1.5: The structure of an actin monomer. Orange parts mark the DNase I-binding loop (the Ser14 and methylated His73 loops) and the hinge between domains. Two clefts are formed between the 4 subdomains: the nucleotide- and targetbinding clefts. The interaction in the target-binding cleft often involves an $\alpha$-helix (marked in magenta). The figure was modified from [28].

\subsubsection{Polymerization}

The gene encoding actin can be traced back to 3 billion years. Almost all eukaryotic cells carry genes encoding actin (except the Nematode sperm). Actin is the most abundant protein family inside cells (the concentration can reach around $1 \mathrm{mM}$ in muscle cells). Actin filaments assemble to provide forces for endocytosis, blebbing and cell movement (Figure 1.4A and D). Myosin motors can use actin filaments as tracks to transport organelles as well as to produce contraction force to enable the separation of two daughter cells during cytokinesis (Figure 1.4B and C) The ubiquitous functions of actin make it essential for the survival of cells [27].

Monomeric actin (or global actin, G-actin) is a 375-amino-acid polypeptide chain folded into 4 subdomains (Figure 1.5): subdomain $1 \& 2$ form the $\alpha$ domain and subdomain $3 \& 4$ form the $\beta$ domain. Two clefts are formed between the domains due to the little contact with $\alpha$ and $\beta$ domain. The upper cleft binds the nucleotide and the lower cleft binds another G-actin or other actin binding proteins [28]. G-actin can grow into an actin filament (filamentous or F-actin). As a dimer or a tirmer of actin is unstable, nucleation is very slow. Actin filaments can grow rapidly once stable trimeric nuclei form (Figure 1.6). Due to the asymmetric structure of G-actin, F-actin 
is polar, with one end called the barbed end and the other called the pointed end. The barbed end containing ATP grows faster and orients toward the cell membrane. The fresh growing F-actins further push the membrane forward.

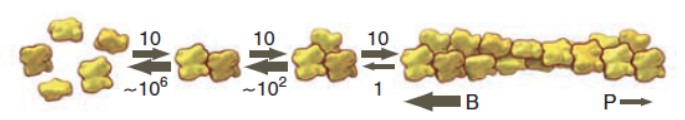

Figure 1.6: Formation of actin filaments: nucleation and extension. To grow from monomeric actin is very difficult and slow. Even dimers and trimers are unstable. However, the elongation by adding new monomeric actin on to the existing nucleus is rapid and stable. The growth is much faster at the barbed end than the pointed end. The figure is modified from [27].

\subsubsection{Depolymerization}

The hydrolysis of ATP by F-actin changes the confirmation and leads to disassembly: the Ser14 $\beta$-hairpin loop forms an hydrogen bond with the $\gamma$-phosphate in ATP. After ATP hydrolysis, the $\gamma$-phosphate is released, Ser14 changes orientation to form a contact with the $\beta$-phosphate and the His73 loop move toward the nucleotide to occupy the space released the $\gamma$-phosphate [28]. Both the barbed end and the pointed end can grow and disassemble. Figure 1.7 shows the association and dissociation rate constants as well as the equilibrium constants $\mathrm{K}^{1}$ of both the F-actin ends. As the equilibrium constant determines the critical concentration for actin polymerization, the difference in the equilibrium constatnt for ATP-actin at the barbed end $(K=0.12)$ and the pointed end $(\mathrm{K}=0.6)$ causes growth on the barbed end and depolymerization on the pointed end - known as treadmilling.

\subsubsection{Role of actin binding proteins}

Actin dynamics requires the regulation of a variety of proteins. Figure 1.8 shows how an actin network forms a dendritic structure via the treadmilling process. We first introduce the essential proteins shown here in detail and then talk about two other proteins (Aip1 and coronin) that help in depolymerization.

Besides elongating the existing F-actin, new filaments can grow on the side of existing filaments to form branches. The nucleation of a branch is initiated by active Arp2/3, a protein complex composed of seven subunits. Usually less than $1 \%$ of Arp2/3 is in the active form [30]. In the inactive form, the Arp2/3 complex is loosely

\footnotetext{
${ }^{1}$ the ratio between dissociation and association rate constant $(\mathrm{k}-/ \mathrm{k}+)$.
} 


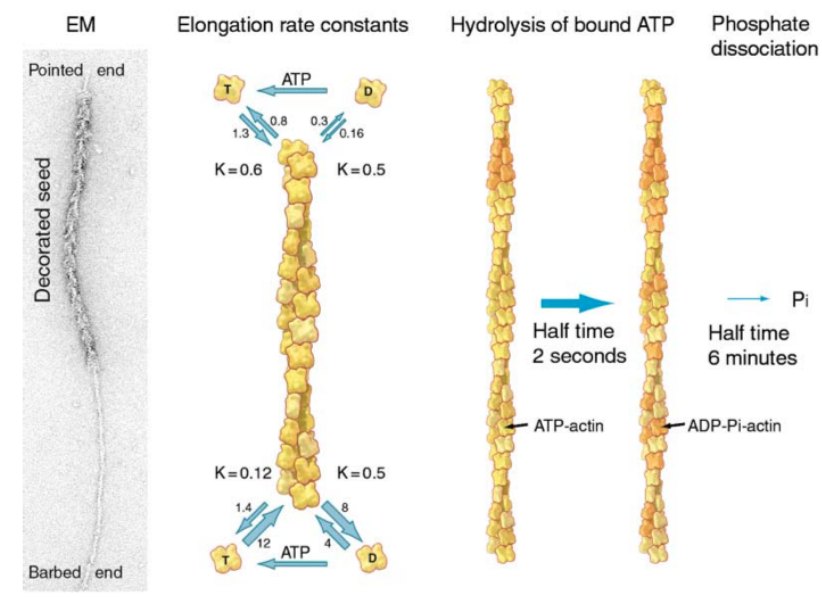

Figure 1.7: Actin filament elongation and ATP hydrolysis. The unit of association rate constants are $\mu \mathrm{M}^{-1} \mathrm{~s}^{-1}$. The unit of dissociation rate constants are $\mathrm{s}^{-1}$. The ratio of the dissociation rate constant to the association rate constant is $\mathrm{K}$, the dissociation equilibrium constant with units of $\mu \mathrm{M}$. Reprinted from [29], Copyright (2003), with permission from Elsevier.

packed. Together with actin filaments and actin monomers, regulating proteins called nucleation promoting factors (NPFs) such as WASp and Scar, Arp2/3 can be activated by a conformational change: all subunits are brought together to form a compact structure wrapping around the helix of actin filament [31]. It is the coorperation of NPFs, actin filaments, actin monomers that activates Arp2/3 and then initiates the formation of new branches. None of them can activate Arp $2 / 3$ on their own. The binding of the activated Arp2/3 to the mother filaments thus forms branch junctions and the barbed end of a new filament (known as a daughter filament) can grow from here. The angle between these two filaments is $70^{\circ}[32,33]$.

The keratocyte and $D$. discoideum cells can move their body length $(10 \mu \mathrm{m})$ in one minute. However, pure actin filaments are intrinsically stable: exchange of subunits at the ends is around $0.04 \mu \mathrm{m} / \mathrm{min}$ [29]. Therefore, it is the role of actin binding proteins that enables the rapid turnover of actin networks. Rapid turnover requires an abundant pool of actin monomers, efficient polymerization and fast deploymerization to replenish the pool of actin monomers. Under physiological conditions, the unpolymerized pool of G-actin usually has 2 to 3 orders of magnitude more actin monomers than the critical concentration ${ }^{2}$ (though it varies among different species) (Figure 1.9). However, not all G-actin can be directly polymerized to F-actin.

${ }^{2}$ Critical concentration here is defined as the equilibrium dissociation constant $\left(\mathrm{K}_{\mathrm{d}}\right)$. Under physiological conditions, $\mathrm{K}_{\mathrm{d}}$ for ATP-actin is $0.1 \mu \mathrm{M}$ at barbed-end and $0.6 \mu \mathrm{M}$ at pointed-end [34]. 


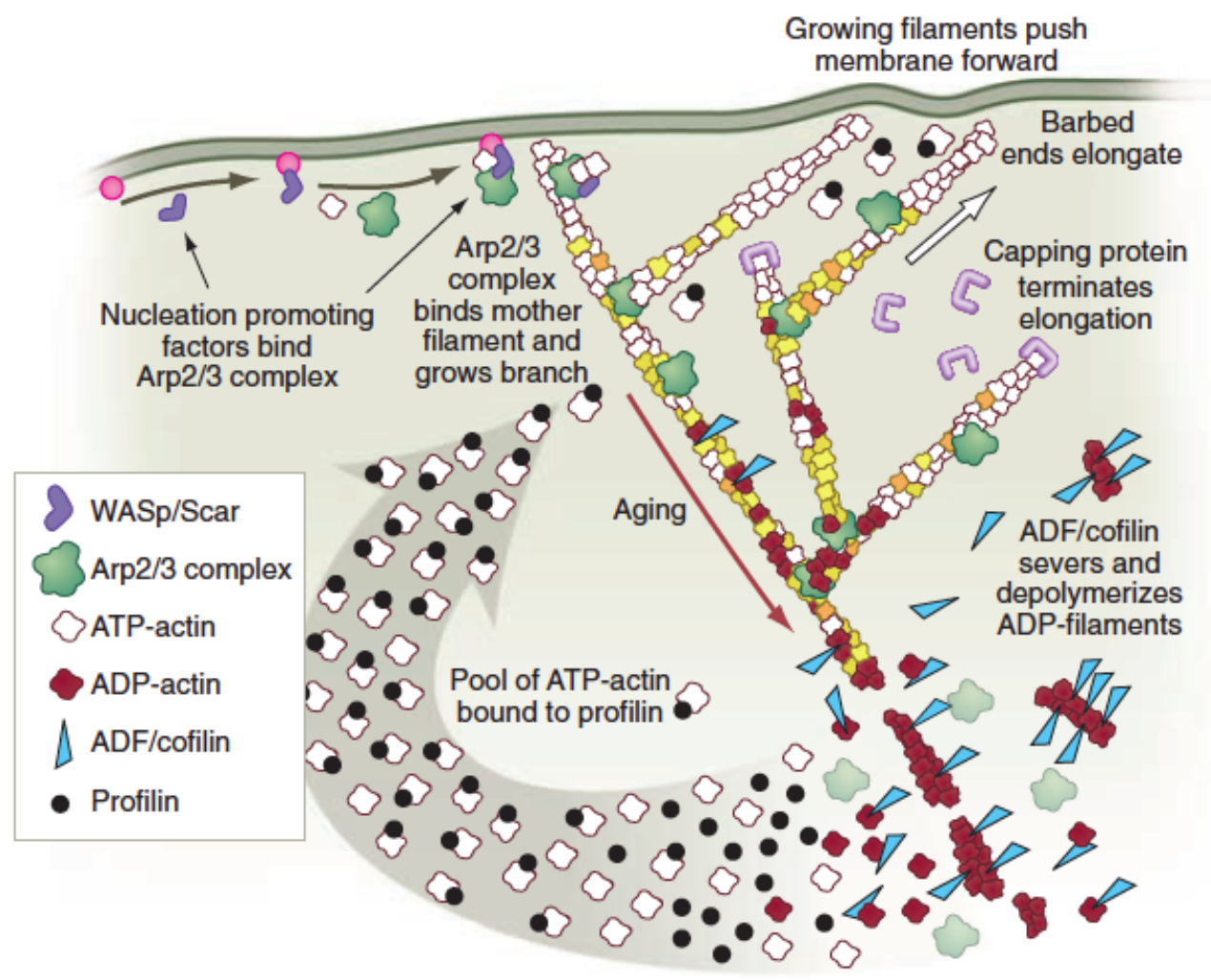

Figure 1.8: Formation of dendritic actin network and the key regulation proteins. Nucleation promoting factors like WASp/Scar bring Arp2/3 and an actin monomer together to the side of an existing filament to form a branch. The pool of ATP-actin bound to profilin bind to barbed ends to elongate the actin filament. Elongation is terminated by the binding of a capping protein. The ATP-actin on the actin filament is gradually hydrolyzed into ADP-actin (aging). ADF/cofilin can sever and dissociate ADP-actin filaments. The ADP-actin then rapidly exchanges a nucleotide to become ATP-actin with the help of Profilin. The profilin bound ATP-actin is then again ready to elongate barbed ends. The growing filaments can push the membrane forward. The figure was modified from [27] 
TABLE 1 Cellular concentrations $(\mu \mathrm{M})$ of key proteins in the actin system of diverse cells

\begin{tabular}{llllcll}
\hline Protein & Acanthamoeba & Dictyostellium & $\begin{array}{l}\text { Neutrophil } \\
\text { unactivated }\end{array}$ & $\begin{array}{l}\text { Xenopus } \\
\text { cgg extract }\end{array}$ & $\begin{array}{l}\text { Platelet } \\
\text { unactivated }\end{array}$ & S. cerevisiae \\
\hline Polymerized actin & 100 & 90 & 100 & 4 & 330 & 2 \\
Unpolymerized actin & 100 & 160 & 300 & 12 & 220 & 0.01 \\
Profilin & 100 & & & 5 & & present \\
Thymosin- $\beta 4$ & $?$ absent & ? absent & & 20 & 550 & absent \\
ADF/cofilin & 20 & $<100$ & & 3 & 30 & present \\
Arp2/3 complex & $2-4$ & present & 10 & & 9 & present \\
Capping protein & 1 & 1 & $1-2$ & & 5 & 1 \\
Gelsolin & $?$ absent & & & & 5 & \\
$\alpha$-actinin & 4 & 3 & & & & \\
Filamin & $?$ absent & & & & & \\
ABP120 & $?$ absent & 6 & & & & \\
\hline
\end{tabular}

References: Acanthamoeba (43, 134, 30, 58, 106); Dictyostelium (John Condeelis, Albert Einstein College of Medicine); neutrophil (32, Cano 91, 38, 50); platelet (John Hartwig, Harvard Medical School, 87, 9, 46); S. cerevisiae (John Cooper, Washington University).

Figure 1.9: Key actin related proteins in different cells. The table was adapted from [34]

Profilin and Thymosin- $\beta 4$ compete with each other to bind ATP-actin monomers. Thymosin- $\beta 4$ cannot participate in actin filament nucleation or elongation, whereas profilin helps to effectively add subunits to F-actin. Profilin can exchange ADP for ATP on G-actin and then add subunits only to the barbed ends (but cannot add them to the pointed ends).

As G-actin is abundant and Profilin can only elongate the barbed end of F-actin, the amount of F-actin itself determines the growth of F-actin. Profilin can also inhibit filament nucleation to prevent elongation along the new F-actin. To terminate polymerization, a capping protein binds tightly on the barbed end with extremely slow dissociation rates ${ }^{3}: 5 \times 10^{-4} \mathrm{~s}^{-1}$; on the other hand, binding of $\mathrm{ADF} /$ cofilin to ADP-actin monomers changes the twist of actin helix and promotes severing of the filaments into short segments. It is the higher affinity of ADF/cofilin for ADPactin monomers that drives the severing process; ADP-actin very quickly turns into ATP-actin due to the high concentration of ATP in living cells; profilin can bind to ATP-actin and polymerize the filaments again owing to higher affinity of profilin for ATP-actin than ADF/cofilin. Capping proteins and cofilin are of extreme importance to cell as lacking the associated regulating genes are lethal to cells [35, 36].

${ }^{3}$ The half-time of uncapping is $>1000 \mathrm{~s}$, much longer than the lifetime of actin dynamics (tens of seconds). 
Besides cofilin, proteins such as Aip1 (actin-interacting protein 1) and coronin also help regulate actin depolymerization. Aip1 can not only enhance fragmentation of actin filaments mediated by cofilin [37-40] but also cap the barbed ends of those severed fragments [41, 42]. Aip1 translocates dynamically to regions that are enriched in filamentous actin (such as pseudopodia, lammellipodia and phagocytic cups) [43-45]. Coronin is named after its localization in crownlike extensions of cell surfaces [46]. Coronin plays two different roles in the front and the back of the cell. At the front of the cell, coronin not only binds to the ATP-actin filaments with high affinity [47-54] but also recruits Arp2/3 complex to the sides of filaments to form new nucleation sites and branch [53, 55-57]. Besides promoting the growth of actin filaments, coronin can inhibit depolymerization in the front as well. The coiled-coil domain of coronin has high affinity toward ATP-actin. Coronin can thus bind ATP-actin to block cofilin binding and prevent the severing of ATP-actin filaments. On the contrary, at the rear of the cell, the coiled-coil domain no longer inhibits the binding of cofilin but allows the $\beta$ - propeller domain to recruit cofilin to disassemble and sever actin filaments $[52,58]$. After the disassembly of actin filaments, Arp2/3 complex and coronin dissociate and then diffuse to the front of the cell. Taken together, the mechanism regenerates fresh actin monomer pool to supply the rapid turnover of moving cells.

Both Aip1 and coronin are important for cytokinesis, development and movement. In cells lacking either Aip1 or coronin, cytokinesis is prolonged and cells are usually multinucleated. The reduced endocytosis and phagocytosis rates in Aip1-null or coronin-null cells result in slower growth rates [45, 58, 59].

\subsection{Myosin II}

To date, 13 types of myosin have been discovered from the genome sequence of D. discoideum cells [26]. Figure 1.10 shows the conceptional structure of them [60]. Based on the structure, myosin can be categorized into several types. Most myosins belong to class II (from now on we will call it myosin II). Myosin II is also the protein responsible for the contration in cardiac, skeletal and smooth muscles. Myosin II, due to its significance, is well studied and known as conventional myosin. The other types of myosin are thus called unconventional myosin. Myosin II molecules with similar function and structure as their conterparts in muscle are are present in all non-muscle eukaryotic cells [61]. We thus call them non-muscle myosin. In this study, we will focus on the non-muscle myosin in $D$. discoideum cells. 
Chapter 1. Introduction

\begin{tabular}{|c|c|}
\hline Name & Domain Structure \\
\hline \multicolumn{2}{|c|}{ Class I } \\
\hline myoA & motor ${ }_{\text {S }}^{10}$ TH1 - \\
\hline myoB & $\mathrm{S}_{\mathrm{S}} \mathrm{IO}$ \\
\hline myoC & motor 10 TH1 $1 \mathrm{TH2}-\mathrm{SH}_{3}-$ \\
\hline myoD & motor ${ }_{S}^{10}$ TH1 THSH32. \\
\hline myoE & motor $\int_{S}^{10}(\mathrm{TH} 1-$ \\
\hline myoF & 5 \\
\hline myoK & TH2 \\
\hline \multicolumn{2}{|c|}{ Class II } \\
\hline mheA & \\
\hline \multicolumn{2}{|c|}{ Class VII } \\
\hline myoI & motor Ias TH4 TH4 THA \\
\hline
\end{tabular}

\begin{tabular}{|l|l|}
\hline \multicolumn{2}{|l|}{ Class V or XI } \\
\hline myoJ & motor las $\frac{\mathrm{c}}{2}$ \\
\hline myoH & \\
\hline New Class? & \\
\hline myoM & motor las \\
\hline
\end{tabular}

Figure 1.10: Schematic representation of the myosins in $D$. discoideum. The table lists the name and domain structure for each $D$. discoideum myosin. Reprinted from [60], Copyright (2001), with permission from Elsevier. 


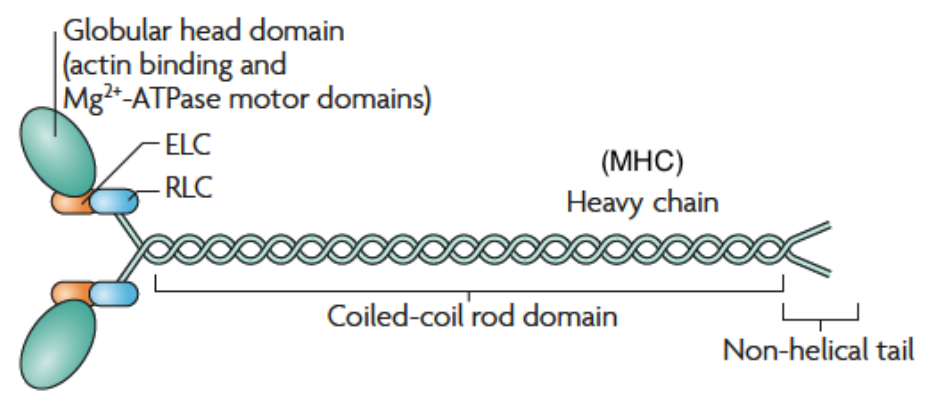

Figure 1.11: The structure of non-muscle myosin II. The global head domain binds to the actin filament. With the hydrolysis of ATP, myosin II can move on actin filament so it is also regarded as motor domain. The regulatory light chains (RLC) can regulate the activity of myosin II and the essential light chains (ELC) can stabilize heavy chain. They bind to the heavy chains at the neck part that link the head and tail domains. Adapted by permission from Macmillan Publishers Ltd: Nature Reviews Molecular Cell Biology [61], copyright (2009)

Myosin II is a hexamer composed of two myosin heavy chains (MHC), two regulatory light chains and two essential light chains (Figure 1.11). Each subunit is encoded by a single gene. The heavy chain can be divided into a globular head domain that binds to actin filaments, a neck region that binds two light chains and a long tail domain. The light chains are essential to stabilize the neck region and make the neck a rigid lever arm that can swing on an actin filament (Figure 1.12) [62, 63]. Myosin II can self-assemble via the long heavy chain tail to form bipolar filaments: two myosin II tails minimize the electrostatic interactions of repeating hydrophobic and alternating negtively and positively charged residues in the tail domain by staggering 14 residues and forming a coiled-coil structure [64]. With electrostatic interactions, the hexamer myosin II can further form parallel dimers, antiparallel tetramers and grow thicker by lateral addition of parallel dimers [65]. Immunoelectron microscopy shows that myosin II bipolar filaments are composed of 10-20 molecules and are about $0.5 \mu \mathrm{m}$ long and $12 \mathrm{~nm}$ thick in $D$. discoideum cells [66]. Since the electrostatic forces hold bipolar filaments together, the formation of myosin II filament highly depends on the ionic strength.

Besides ionic strength, phosphorylation of the heavy chain also regulates the assembly of myosin II filament. Myosin heavy chain kinase A (MHCK A) can phosphorylate threonine at positions 1823, 1833 and 2029. The bending structure of phosphorylated myosin heavy chain observed from electron micrographs postulates that due to the stabilization from the negative phosphate groups and the positively charged 


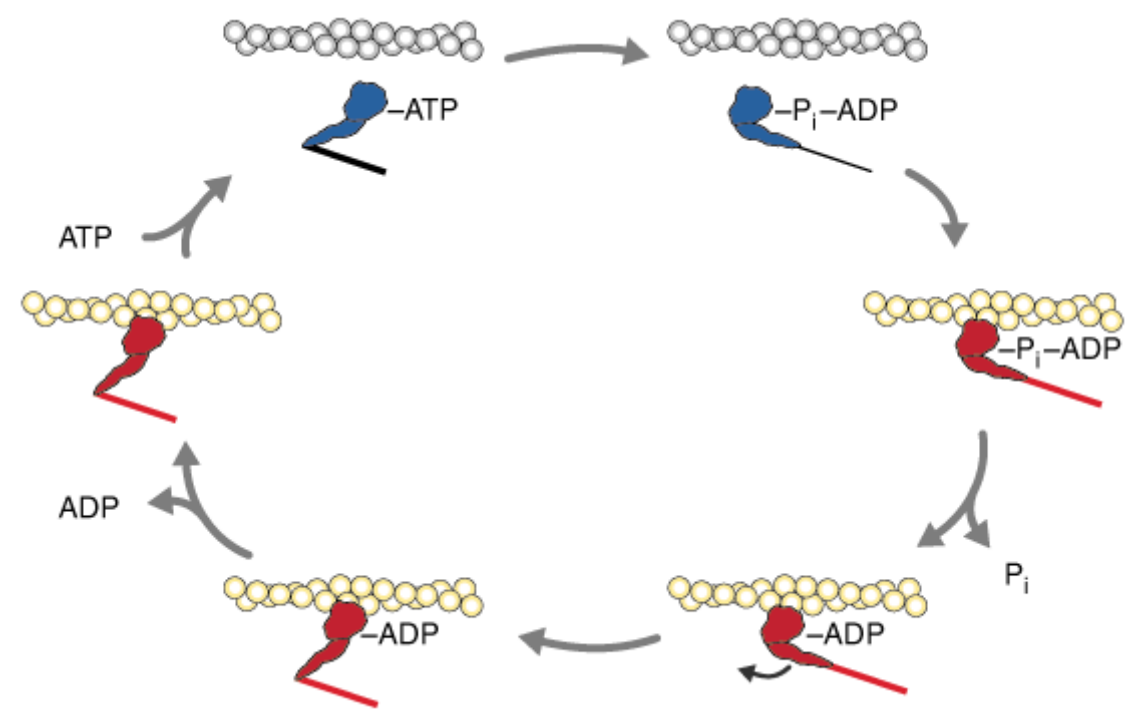

Figure 1.12: Schematic diagram to show the interaction between actin and myosin and the corresponding ATPase cycle of myosin. Myosin in red shows the bound state to actin filament, whereas myosin in blue shows the detached state. Adapted by permission from Macmillan Publishers Ltd: [Nature Cell Biology] [63], copyright (2001)

residues, myosin II monomers can form folded structure (Folded monomers in Figure 1.13) and inhibit the assembly of bipolar filaments [67, 68]. The motor domain enables myosin II to bind on actin filaments and form actomyosin. By hydrolyzing ATP, myosin II motor head can slide on actin filaments and induce contraction (Figure 1.14) [69]. In Figure 1.12, the ATPase cycle of myosin shows that myosin first hydrolyzes ATP without binding to actin. The following attachment to actin triggers the release of phosphate and induces the force-generating stroke [70, 71]. The tight coupling between hydrolysis of ATP and the interaction of actin-myosin prevents unnecessary ATP consumption [63]. As myosin II is only functional as filaments, the lack of myosin II heavy chain thus inhibits the entire function of the myosin II molecule[61].

Myosin II plays an important role in cell migration, cell shape maintance, and cytokinesis: regarding the first role, in a migrating cell, actin filaments polymerize in the front and push the membrane forward; meanwhile myosin II accumulates in the rear and leads to contractile forces. In response to a chemoattractant, the localization of myosin II can surpress lateral pseudopod formation to maintain the polarity of the cells, resulting in persistent movement towards the chemoattractant. In the absence 


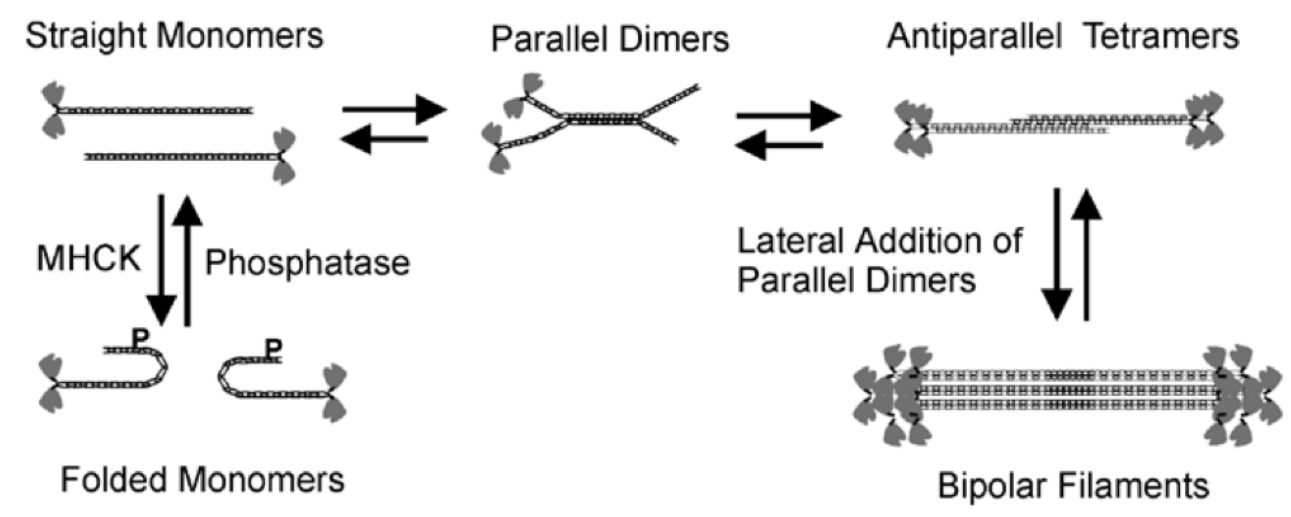

Figure 1.13: Assembly pathways of myosin II filament in $D$. discoideum. Myosin heavy chain kinases phosphorylate the tail of myosin II resulting in the folding of myosin II and thus prevent filament assembly. Myosin II monomers can assemble into parallel dimers and antiparallel tetramers. The lateral addition of parallel dimers composed of tetramer can form biopolar filaments. The figure is adapted from [65]

of myosin II, cells are still able to move albeit at slower speed; they are less polarized and unable to effectively direct towards a chemoattractant. Secondly, actomyosin filaments can support the membrane and maintain cortical tension. A D. discoideum cells without myosin II cannot squeeze itself to pass through a layer of agarose toward cAMP source but fragement instead [72]. As cell-cell contact becomes important in the aggregation stage, the inability to sustain a force prevents the development into a fruiting body. Thirdly, myosin II accumulates in the cleavage furrow of dividing cells, where a contractile ring is formed, to assist cytokinesis (Figure 1.4). Cells lacking myosin II are usually multinuclated due to their incapability to divide in suspension. [73] 


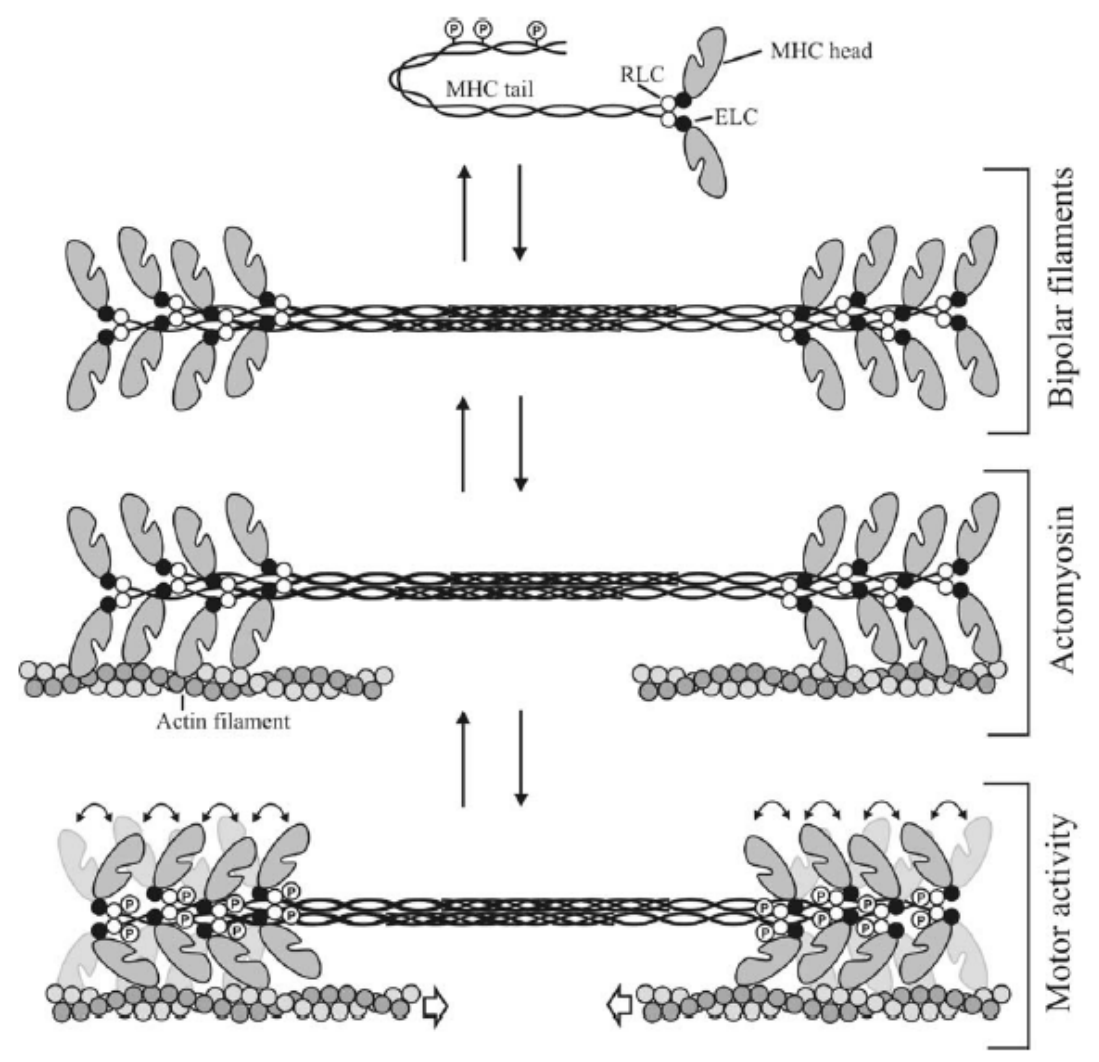

Figure 1.14: Myosin II is a hexamer composed of two regulatory light chains (RLC), two essential light chains (ELC) and two myosin heavy chains (MHC). Each heavy chain has a head domain and a tail domain. Myosin heavy chain kinases (MHCKs) can phosphorylate three threonines of the tail to prevent the assembly of myosin II filaments. Actomyosin refers to the structure formed when myosin II binds to actin filaments via the interaction of motor domain. With the hydrolysis of ATP, myosin can show its motor activity. Reprinted from [69], Copyright (2006), with permission from Elsevier. 


\subsection{Self-organization and oscillations}

Self-organization is a process where global order emerges from local interactions between the components of a disordered system. Schools of fish, flocks of birds, and patterns on animal fur are classic examples of biological pattern formation driven by self-organization. Self-organization also plays an important role in the structure formation of a cell. The local dynamics of actin filaments enables cell movement by forming pseudopodia, blebbs, filopodia, membrane ruffling and other structures. It is the self-organization of various molecules inside the cell that determines the dynamic behavior of cells.

One of the well-known examples is the actin comet tail of the bacteria Listeria monocytogenes. It uses the actin system of the cell it infects to propel itself within the cytoplasm of the host as well as to invade adjacent cells. The actin tail initiates from the asymmetric distribution of the protein ActA on the surface of Listeria. ActA protein not only interacts with monomeric ATP-actin, but also activates and recruits anchoring proteins such as Arp2/3 and VASP to support elongation. The insertion of actin thus produces force for propulsion [74-78]. Another intriguing example of cytoskeletal self-organization are actin waves, which propagate at the substratedattached surface by constantly polymerizing in the front and depolymerizing in the back. It was proposed that $D$. discoideum cells use actin wave to scan the surface for particles to intake [79]. The three-dimentional ordered self-organization follows the signal of PIP3 and is operated by actin binding proteins: Arp2/3 is associated with actin throughout the wave, whereas myosin IB is enriched in the front and coronin is recruited at the back for disassembly [80-83].

In particular cases, collective behavior of many interacting components can result in systems with an intrinsic potential to oscillate. Oscillatory systems are known to play important roles in biology $[84,85]$ : circadian clocks circle between day and night to coordinate behavior with daily and seasonal changes [86-88], Min protein systems recycle inside Escherichia coli to determine the division site $[89,90]$ and cilia beat to sense factors such as frequency of mechanical sources in the environment [91, 92]. The most well-known oscillation of $D$. discoideum cells is the periodic emission of cAMP (with a period around $6 \mathrm{~min}$ ) during their starvation stage, which enables them to stimulate each other and then accumulate to develop into fruiting bodies [93, 94]. The periodicity manifested itself in experiments such as (1) light scattering by cells observed by dark-field microscopy (2) contact among cells observed by brightfield microscopy and (3) cell-surface contact observed by total internal reflection 
fluorescence microscopy (TIRFM) [95, 96]. In addition, cAMP also triggers the uptake of calcium, resulting in oscillations of $\mathrm{Ca}^{2+}[97,98]$.

Besides the oscillations of cAMP, oscillations of several different components have been found in D. discoideum in the absence of a chemoattractant: PIP3 (PtdIns 3,4,5trisphosphate) has been found to spontaneously oscillate on the membrane with a period of around $200 \mathrm{~s}[99,100]$; protrusion of a pseudopod formed with a frequency of around 3.5/min [101-108]; SCAR/WAVE, coronin and LimE were recently found to oscillate with a period of around $10 \mathrm{~s}[109,110]$.

\subsection{Aim and outline}

D. discoideum shares many common features of actin dynamics and essential responses with eukaryotic cells and cancer cells. Therefore, most properties of the oscillations are well studied in the simpler biological model system. However, the properties and the underlying mechanisms of the recently found autonomous cytoskeletal oscillations are still unexpolred. Questions such as what the roles of this autonomous oscillation in chemotaxis are, and how external stimulation affects autonomous oscillations are intriguing but unanswered.

This study will start by investigating actin dynamics of cells in the absence of external stimuli. We analyze thousands of cells to get a stochastically significant mean. In the part on intrinsic oscillations, we report on the properties like the distribution of oscillations, role of actin regulating proteins such as Aip1, coronin and myosin II and propose an underlying mechanism. In the second chapter, we investigated how external stimulation alters the intrinsic frequency. With dose dependence experiments and careful examination of the different sections of the actin polymerization, depolymerization and recovery, we extend our model to account for the chemotatic responses. We also use the experimental data to verify an existing model of actin dynamics and modify it according to our experimental results. Finally, we investigate the role of myosin II in actin dynamics. Although myosin II is not directly invovled in actin regulation, it is essential for effective cellular functions such as movement, chemotaxis and cytokinesis. Here we first study the dynamics of myosin II to understand not only how myosin II regulates actin dynamics but also how the self-organized actin network influences the dynamics of myosin II. Myosin II-knockout cells are further studied to reveal the significance of myosin II in regulating the chemotatic relevance in actin dynamics as well as cellular functions. 


\section{CHAPTER 2 \\ Material and methods}

\subsection{Cell culture and development}

Dictyostelium discoideum cells were cryopreserved in vials in the form of spores at $-80^{\circ} \mathrm{C}$ for long-term storage. For mutants that cannot form spores, the cells were direrctly frozen in liquid nitrogen. The first step to grow cells is to thaw the frozen cells. Vials from freezer or liquid nitrogen were first thawed at room temperature. Cells were grown at $22^{\circ} \mathrm{C}$ in HL-5 medium on Petri dishes. To germinate cells from spores, $100 \mu \mathrm{M}$ spores were cultured on a Petri dish with $10 \mathrm{~mL}$ HL-5 medium (14 g/L peptone, $7 \mathrm{~g} / \mathrm{L}$ yeast extract, $13.5 \mathrm{~g} / \mathrm{L}$ glucose, $0.5 \mathrm{~g} / \mathrm{L} \mathrm{KH}_{2} \mathrm{PO}_{4}, 0.5 \mathrm{~g} / \mathrm{L}$ $\mathrm{Na}_{2} \mathrm{HPO}_{4}$, Formedium, Norwich, England). To obtain cells from frozen cells, the cryopreservation medium containing DMSO was first replaced by fresh HL-5 medium and then put into a Petri dish.

To be free of asepsis, all the reagents (e.g. buffer and medium), glassware, disposable plastic wares were sterilized. The working area and the exterior of equipment were always cleaned with $70 \%$ ethanol. Depending on the cell lines, antibiotics were supplemented as selection markers to protect the integrity of cell culture from the second day of thawing. Figure 2.1 shows the growth of AX2-LimEGFP cell from the first day of thawing. After the cells occupied all the available substrate on the Petri dish (i.e., reached confluence, Figure 2.1D), we subcultured the cells by transferring some of the cells to a new Petri dish with fresh medium to grow (i.e., a new passage). Every subculture step increased the passage number of cells by one. Depending on the density and growth rate of different cell lines, the cell culture was passaged within 3 days. Since cell lines in continuous cultures are likely to be genetically unstable as the passage number increases, cells were discarded when the passage number reached 15 . 


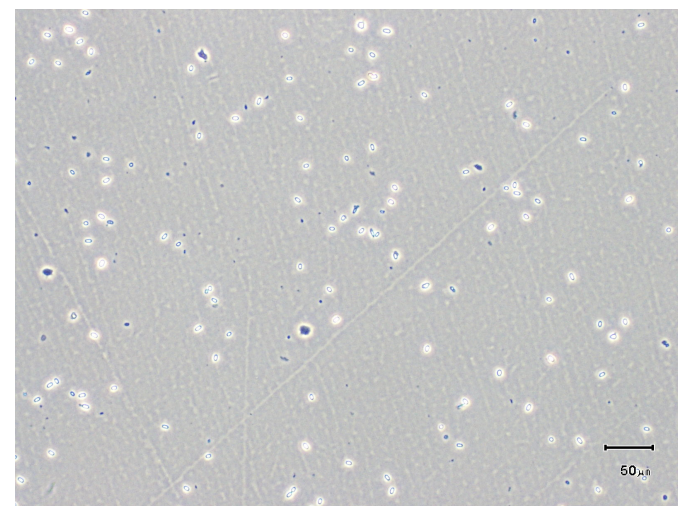

(A) day 1

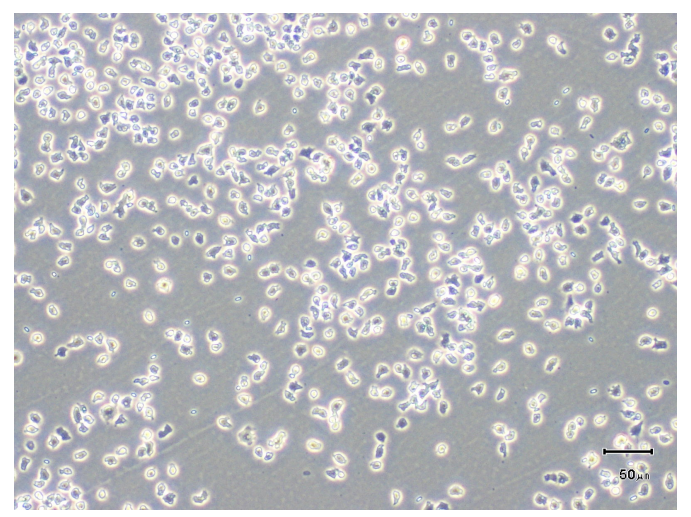

(C) day6

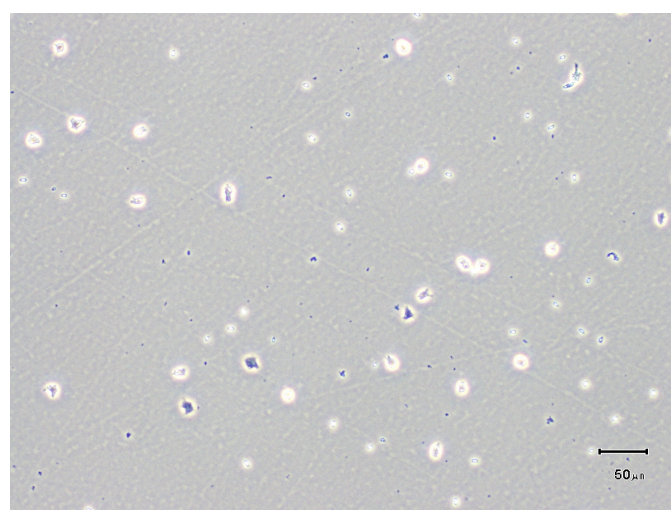

(B) day3

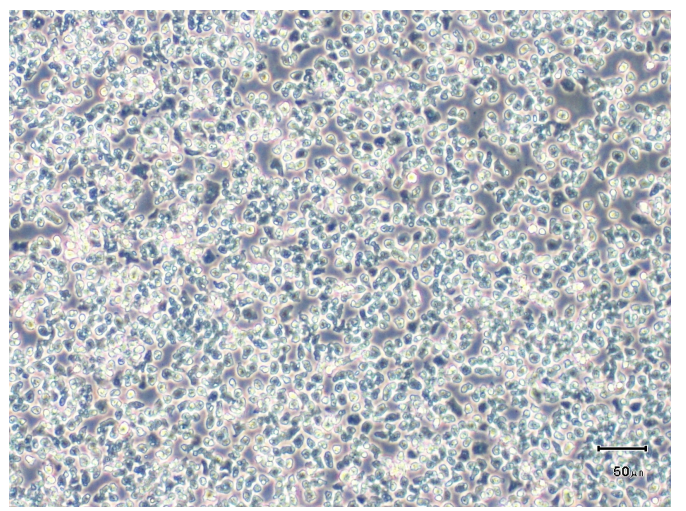

(D) day7

Figure 2.1: The growth of AX2-LimE-GFP cells on a Petri dish.

Due to the genetic instability of cells with high passage number, the cells were cryopreserved in the form of cells or spores once there was a surplus of cells at very early passage numbers. To freeze spores, the cells were first washed out from the Petri dishes and HL-5 medium was completely replaced with phosphate buffer (PB, $\mathrm{KH}_{2} \mathrm{PO}_{4} 2 \mathrm{~g} / \mathrm{L}, \mathrm{Na}_{2} \mathrm{HPO}_{4} \cdot 2 \mathrm{H}_{2} \mathrm{O} 2 \mathrm{~g} / \mathrm{L}, \mathrm{pH}=6$ ) to ensure the starvation of cells. Next, the starved cells were put onto a Petri dish with a layer of agar made from PB to start the developmental cycle. This Petri dish was then placed in a closed box with a wet tissue to provide moisture. After the formation of spores, they were washed out from the phosphate-agar-Petri dish with PB and dispensed aliquots of the spore suspension into cryogenic storage vials. These vials were put into liquid nitrogen for $1 \mathrm{~min}$ and then stored at $-80^{\circ} \mathrm{C}$. For mutant cells that cannot form spores, cells were cryopreserved in the form of cells. Cells were first washed out from Petri dishes with HL-5, centrifuged to remove the supernatant and then medium A (50\% Fetal 
calf serum (FCS, Invitrogen), 50\% HL-5) was added to dispense the cells. These cells were counted with a hemocytometer and then diluted with 50\% medium A and $50 \%$ medium B (40\% medium A, 40\% FCS, 20\% DMSO as a cryoprotective agent) in order to be aliquoted with a concentration of $10^{7}$ cells $/ \mathrm{mL}$. The aliquoted cells in cryovials were then put into a controlled freezing rate apparatus (Mr. Frosty ${ }^{\mathrm{TM}}$ Freezing Container) to cool at the rate of $-1{ }^{\circ} \mathrm{C} / \mathrm{min}$. This optimal cooling rate for cell preservation was achieved by filling the container chamber with 100\% isopropyl alcohol. The freezing apparatus with cryovial containing cells was first stored at $-20^{\circ} \mathrm{C}$ for $1-2$ hours and then $-80^{\circ} \mathrm{C}$ overnight. Finally the frozen cells were transferred to and stored in liquid nitrogen.

\subsection{Microfluidics}

The microfluidic channels used in the experiments were fabricated using soft lithography. A master wafer was first made by photolithography and then ploy(dimethylsiloxane) (PDMS, Sylgard ${ }^{\mathrm{TM}}$ 184, Dow Corning Europe SA, Houdeng-Goegnies, Belgium) was poured over the master to get an elastomeric block. Finally, the patterned block was bound to a glass slide and was ready for use. Figure 2.2 summarizes the steps of fabrication while the next three subchapters outline the details of the procedure.

\subsubsection{Mask Design}

The pattern of the channels were designed with a computer-aided design (CAD) software and then printed on a mask. The mask design was printed on chrome/quartz with a resolution of $1.8 \mu \mathrm{m}$. Figure 2.2 shows the geometry of channels used in the experiments.

\subsubsection{Photoresist}

Photolithography generates patterns on a surface. The surface coated with photoresist is selectively irradiated by UV light through the transparent part of the predesigned mask. After the chemical reaction induced by exposure to UV light changes the properties of the photoresist, the following developing process washes out either the exposed part (positive photoresist) or the unexposed part (negative photoresist). In our case, negative photoresist is used.

The designed mask was then used in photolithography to create the master wafer. The high contrast, epoxy based SU- 8 was used as the photoresist and the procedure 


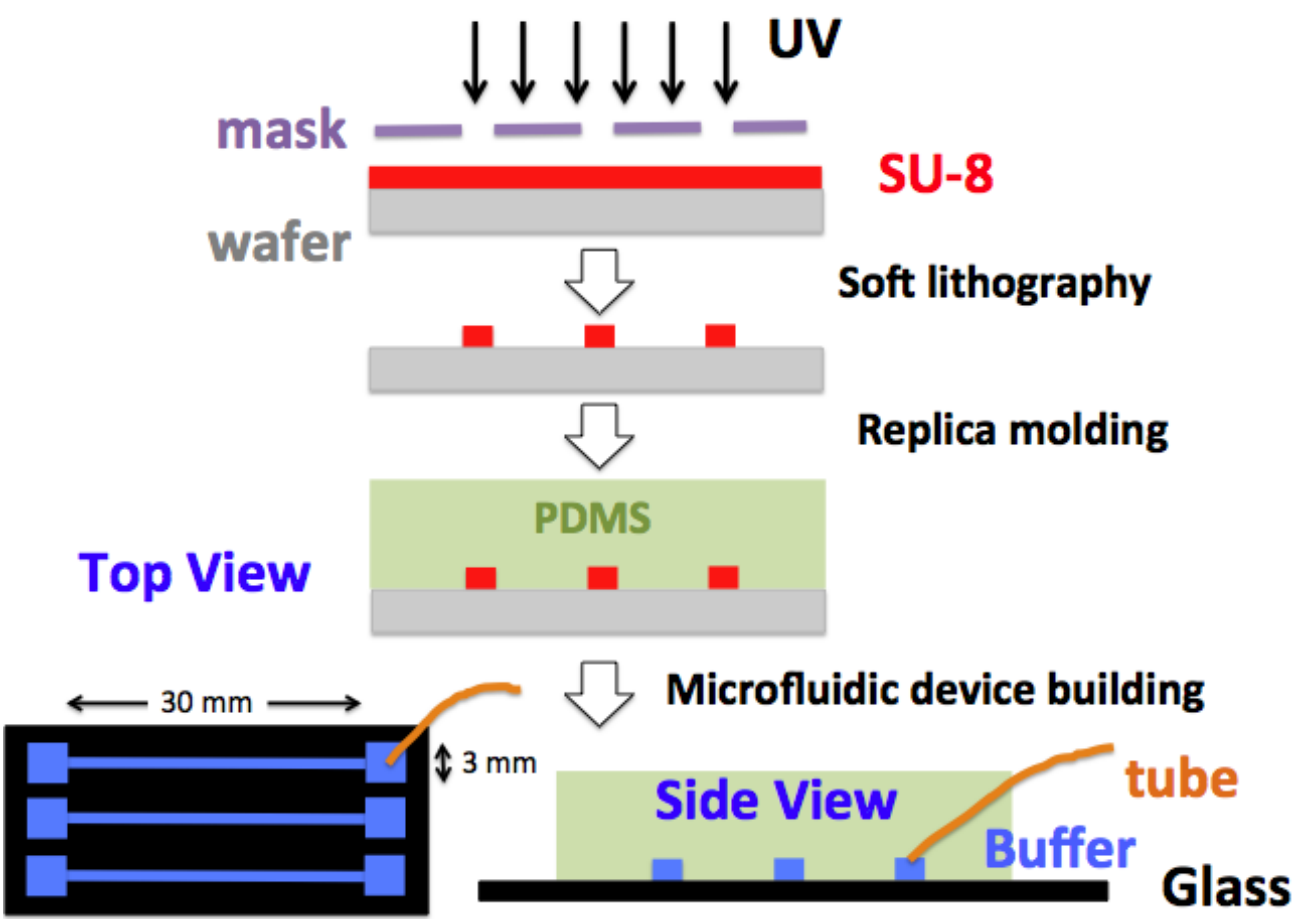

Figure 2.2: Summary of the fabrication of microfluidic channels. Soft lithography removes only the masked part and leaves the designed channels unchanged. PDMS was used for replica molding. Finally, the device is sealed by binding PDMS to a glass cover slide. The geometry of the microfluidic channels used is shown as a top view: channel height: $26 \mu \mathrm{m}$; channel width: $500 \mu \mathrm{m}$.

followed the protocol of MICROCHEM [111]. The process includes wafer cleaning, spin coating, soft baking, UV exposure, post exposure baking, developing, rinsing, drying and finally measuring the height based on interferometry (Figure 2.3).

1. Cleaning. The polished silicon master wafer (diameter $100 \mathrm{~mm}$, SiMat) was first baked at $200^{\circ} \mathrm{C}$ for 5 min to evaporate residual organic solvents on the surface.

2. Spin coating. After the wafer cooled down, it was placed on a spin coater. Approximately $1 \mathrm{ml} \mathrm{SU}-8$ was then poured onto the center of the wafer. The viscosity of SU-8 and the spin speed are vital to the final thickness of the channel. For a channel with a height of $26 \mu \mathrm{m}, \mathrm{SU}-825$ (viscosity=2500 cSt) was used. The coating was done in two steps. The first spread cycle took $5 \mathrm{~s}$ to ramp to $500 \mathrm{rpm}$ with an acceleration of $100 \mathrm{rpm} / \mathrm{s}$. The second spin cycle ramped to $2000 \mathrm{rpm}$ with an acceleration of $300 \mathrm{rpm} / \mathrm{s}$ and was held for a total of $30 \mathrm{~s}$. 
1. Clean

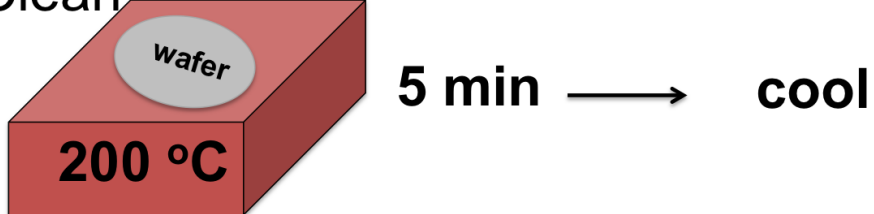

2. Spin coating spin

SU-8 _ - wafer

3. Soft bake

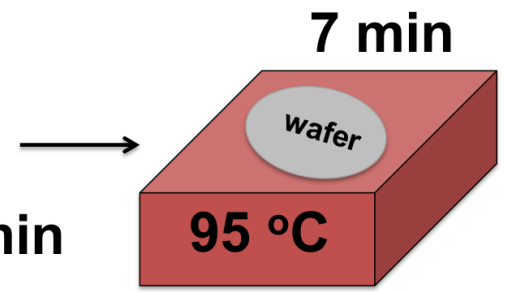

4. UV expose

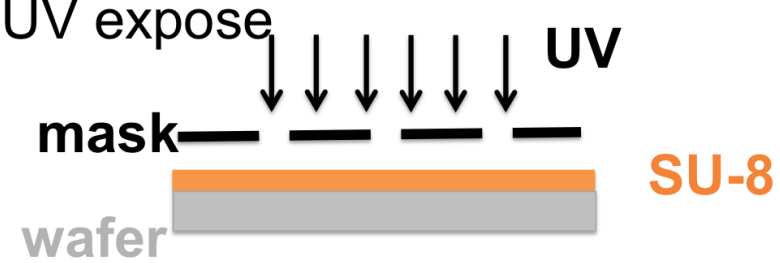

5. PEB

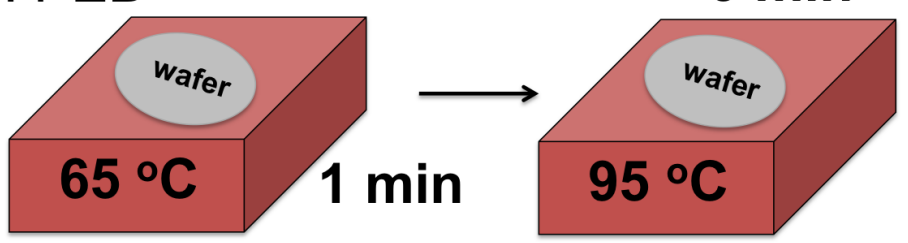

6. Develop

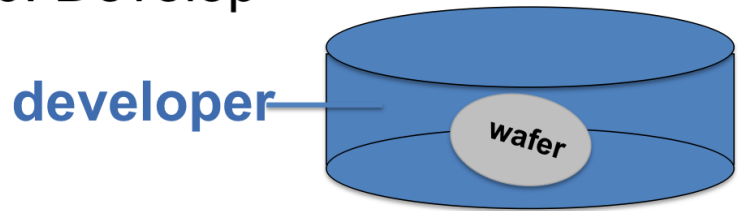

Figure 2.3: Flow chart of photoresist fabrication. The process includes clean, spin coating of SU8, soft bake, UV exposure, post exposure bake (PEB), and finally development. 
3. Soft baking. After the resist spread onto the substrate, the wafer was placed on a hotplate to evaporate the solvents and densify the film. Stepwise heating was used to make the photoresist adhere to the substrate better. The wafer was first heated on a hotplate at $65^{\circ} \mathrm{C}$ for $3 \mathrm{~min}$ and then at $95^{\circ} \mathrm{C}$ for $7 \mathrm{~min}$.

4. UV exposure. The plate was cooled down before exposure to UV. A mercury arc lamp with a power of $350 \mathrm{~W}$ and intensity of $14 \mathrm{~mW} / \mathrm{cm}^{2}$ was used as the light source for $14 \mathrm{~s}$ to provide $200 \mathrm{~J} / \mathrm{cm}^{2}$ exposure energy. The exposure to UV light was carried out in a EVG620 mask alignment system (EVG, St. Florian am Inn, Austria).

5. PEB. The exposure of SU-8 to UV light solidifies the material by cross-linking the long molecular chains. This epoxy cross-linking is acid-initiated and thermally driven. As strong acid was formed during UV exposure, the post exposure baking provides heat for the process of epoxy cross-linking. To reduce the stress resulting from the cross-linking of SU-8, the wafer was first heated on a hotplate at $65^{\circ} \mathrm{C}$ for $1 \mathrm{~min}$, then at $95^{\circ} \mathrm{C}$ for $3 \mathrm{~min}$ and finally gradually cooled down to room temperature.

6. Development. The wafer was then immersed in the developer of SU-8 (1Methoxy-2-propanol acetate) until all non-cross-linked SU-8 was washed out. Finally, we rinse the wafer with isopropyl alcohol and dry it with nitrogen. The height can be verified by white light interferometry (Wyko NT 1100, Veeco, Plainview, NY).

\subsubsection{Soft lithograpghy}

The key to soft lithography is the patterned structures on the surface of an elastomeric block. PDMS is a fluid at room temperature but can be readily converted into solid elastomers by cross-linking. A liquid silicon rubber base (i.e. a vinyl-terminated PDMS) and a curing agent (i.e. a mixture of a platinum complex and copolymers of methylhydrosiloxane and dimethylsiloxane) were first mixed in a 10:1 ratio, degassed in a vacuum desiccator and finally cured for 1 to 2 hours at $75{ }^{\circ} \mathrm{C}$. The hydrosilyation reaction between vinyl groups $\left(\mathrm{SiCH}=\mathrm{CH}_{2}\right)$ and hydrosilane groups $(\mathrm{SiH})$ transformed the liquid into a solid cross-linked elastomer $[112,113]$.

Inlet and outlet holes were punched into the replica mold of PDMS with a $0.75 \mathrm{~mm}$ puncher (Harris Uni-Core ${ }^{\mathrm{TM}}$ ). The PDMS and a cover glass (No.1, $24 \times 60 \mathrm{~mm}$, Menzel Gläser, Braunschweig, Germany) were then placed into a plasma vacuum chamber (PDC 002, Harrick Plasma, Ithaca, NY). The vacuum pump was connected to the outlet of the reaction chamber and air was used as the process gas to generate 
plasma. After a $30 \mathrm{~s}$ treatment with a violet plasma, the PDMS and the cover glass were taken out and bound together. The oxidization of PDMS in air plasma enables etching of hydrocarbons leaving silanol groups $(\mathrm{SiOH})$ on the surface, rendering the surface hydrophilic. Immediate contact of PDMS and cover glass enables an irreversible seal by formation of Si-O-Si covalent bond ${ }^{1}$. The hydrophilic surface after the treatment of plasma also helps the spreading of buffer in microfluidic channels. Finally, the inlet of the microfulidic channel was connected to a a glass syringe (McMaster, Hamilton, Ontario) via a PTFE microtube (Novodirect, Kehl, Germany).

\subsection{Experimental design}

To investigate the actin dynamics of migrating $D$. discoideum cells in the presence and absence of external chemotatic stimuli, we took advantage of the fact that D. discoideum cells respond to cAMP when they are starved, and that the microfluidic devices can be used to apply and remove cAMP with high spatiotemporal precision.

The preparation of cells was started one day previous to the experiment. Cells were first washed out of the Petri dish with HL-5 and then centrifuged to remove the supernatant. The number of cells was estimated by a hemacytometer. Around $5 \times 10^{5}$ cells were then collected in a Erlenmeyer flask with a final volume of $25 \mathrm{~mL}$ and shaken at $150 \mathrm{rpm}$ for one day. To start the starvation, cells in the log phase (where cells proliferate exponentially) were collected from the Erlenmeyer flask and the HL-5 medium was replaced with $\mathrm{PB}$. The process of removing the supernatant after centrifuge was repeated twice to ensure the complete removal of nutrients from cells. Finally, a drop of $50 \mathrm{nM}$ cAMP was applied to the cells every 6 min continuously for 6 hours via a peristaltic pump. The cells were then centrifuged to replace the buffer and resuspended in a final volume of $2 \mathrm{~mL}$ fresh $\mathrm{PB}$.

The interaction between cAMP receptors and cAMP enables $D$. discoideum cells to recognize cAMP. As binding to cAMP causes receptor phosphorylation [114], caged cAMP was designed by esterification of the phosphate moiety (marked yellow in Figure 2.4) with a protecting group (marked green in Figure 2.4). This protecting group is photolabile. Application of a flash of light removes the protecting group and turns the inactive caged cAMP into active cAMP to evoke biological responses $[115,116]$. The equatorial isomers of $[6,7-\mathrm{Bis}$ (carboxymethoxy)coumarin4-yl]methyladenosine-3',5'-cyclic monophosphate was the caged cAMP (Figure 2.4A.

\footnotetext{
${ }^{1}$ The website of harrick plasma provides more details (http://www.harrickplasma.com/applicationsmicrofluidic.php).
} 
(A)<smiles>Nc1ncnc2c1ncn2[C@H]1C[C@@H]2COP(=O)(OCc3cc(=O)oc4cc(OCC(=O)O)c(OCC(=O)O)cc34)O[C@@H]1COP(=O)(O)O2</smiles>

(B)

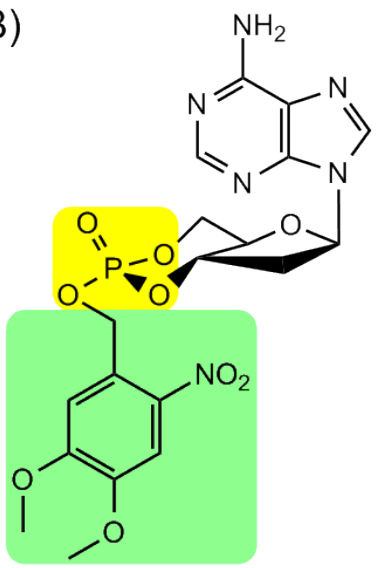

Figure 2.4: Structures of two different types of inactive caged cAMP which can be activate throgugh UV exposure. The phosphate part (marked in yellow) is inactivated by esterification with a photolabile protecting group (marked in green). (A) Structure of BCMCM-caged cAMP molecule. (B) Structure of DMNB-caged cAMP molecule.

BCMCM-caged cAMP, Biolog, Bremen, Germany) used in the experiments. To stimulate $D$. discoideum with the best spatiotemporal resolution, we combined the microfluidic device with flowphotolysis [109, 117, 118]. $10 \mu \mathrm{M}$ BCMCM-caged cAMP was continuously flowed into the microfluidic channel with a flow rate of $120 \pm 6.5 \mu \mathrm{m} / \mathrm{s}$ controlled by a syringe pump (PHD2000, Harvard Apparatus). Each independent experiment was conducted as sketched in Figure 2.5 under a confocal laser scanning microscope. The cells were observed in an imaging region with a size of $48 \times 48 \mu \mathrm{m}$. The $488 \mathrm{~nm}$ line of an Argon laser (Melles Griot, Carlsbad, CA) was used to scan the imaging region. The scanning rate to visualize cells labeled with GFP was $5 \mu \mathrm{s} / \mu \mathrm{m}$. $543 \mathrm{~nm}$ line of a He-Ne laser was used for visualing cells labeled with mRFP at the same scan rate. To record the cell movements, one image was taken every second (recording rate was $1 \mathrm{~Hz}$ ). To stimulate the cell, a $405 \mathrm{~nm}$ laser was scanned over a length of $48 \mu \mathrm{m}$ in the front of the imaging region of the cell with a rate of $0.8 \mu \mathrm{m} / \mu \mathrm{s}$ to uncage BCMCM-caged cAMP.

\subsection{Image processing and data analysis}

All the images recorded from the confocal laser scanning microscope (Olympus Fluoview FV1000) introduced in the following three chapters were first processed and then analyzed with self-written Matlab codes. 


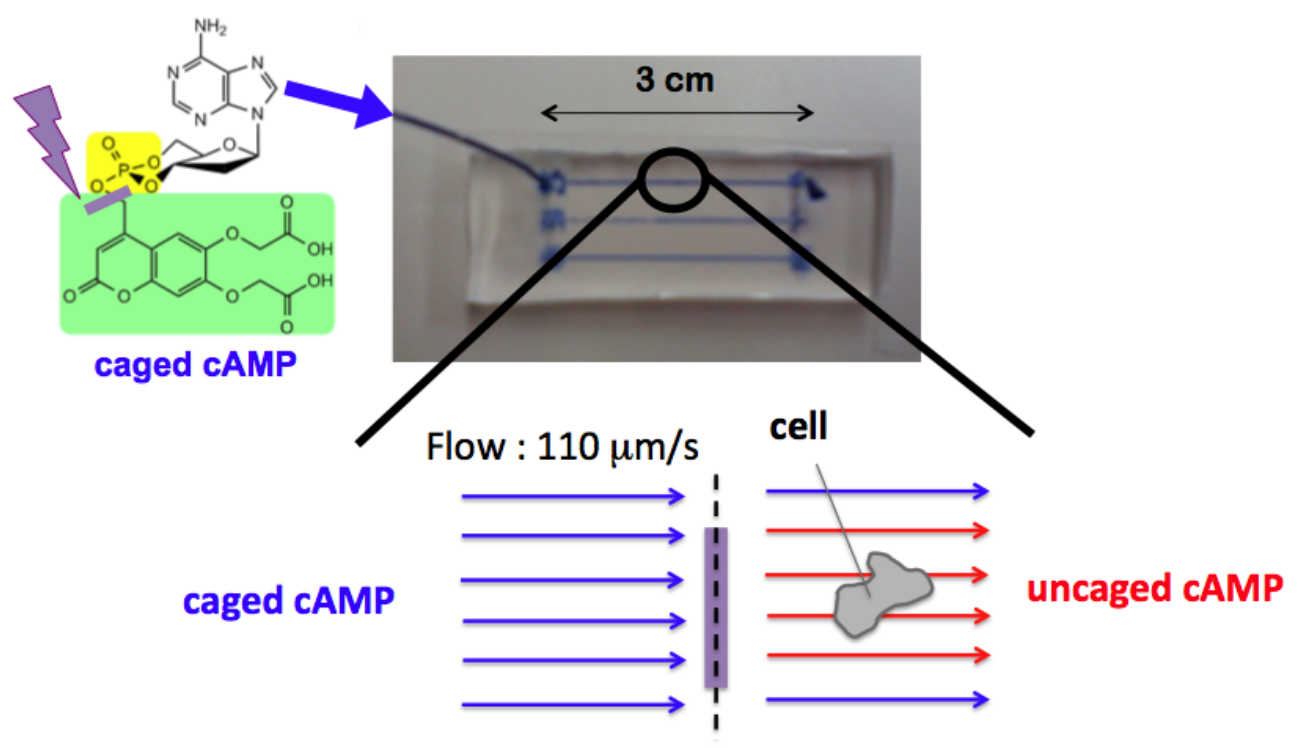

uncaging laser scan

Figure 2.5: A schematic diagram of the experimental setup. BCMCM-caged cAMP was continuously flowed into the microfluidic channel with a rate of $110 \mu \mathrm{m} / \mathrm{s}$. The uncaging laser is always applied right in front of the cell to release functional cAMP.

\subsubsection{Pre-processing filters}

We first remove noise from the fluorescence images by setting a threshold to separate the actual signal from the background noise (binary thresholding). We set the threshold intensity as follows: (1) All pixels from one image (i.e., one cell at one observation time) were classified into one of 10 groups based on their intensity. Group $1(\mathrm{k}=1)$ was composed of pixels with the lowest intensity, whereas the brightest pixels were collected in group $10(\mathrm{k}=10)$. (2) The mean intensity was calculated in each group $\left(<\mathrm{I}_{\mathrm{k}}>\right)$. (3) The intensity differences between adjacent groups were determined $\left(<\mathrm{I}_{\mathrm{k}+1}>-<\mathrm{I}_{\mathrm{k}}>\right.$ ). (4) When the difference between a pair of adjacent groups was significantly larger than others, then the mean of the lower-intensity group was defined as the background threshold. Figure 2.6 shows that the difference between groups 9 and 10 is significantly larger than the rest. So in this case, the mean intensity of the ninth group was taken as threshold.

Secondly, binary thresholding was performed using this calculated threshold. The pixels with intensities higher than the threshold were defined as 1 (plotted as red in Figure 2.7B) and those below the threshold were defined as 0 (plotted as 


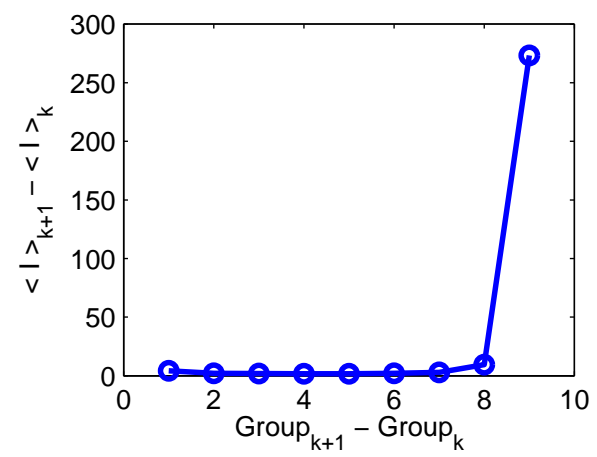

Figure 2.6: Method of finding preliminary threshold. Each pixel from one image (i.e., one cell at one observation time) was classified into 10 group according to its intensity. The intensity differences between adjacent groups were determined $\left(<\mathrm{I}_{\mathrm{k}+1}\right\rangle$ $\left.-<\mathrm{I}_{\mathrm{k}}\right\rangle$ ) and plotted here. Group 9 and Group 10 show distinct difference so the mean intensity of Group 9 was taken as the preliminary threshold.

blue in Figure 2.7B). All the small spots in the background area were then further removed by performing the so-called morphological opening using a disk with the radius of 1 pixel as a structuring element. Figure 2.7C shows the image after the performance of morphological opening ${ }^{2}$ Finally, a median filtering of the image using a 3 pixel-by-3 pixel window was performed to remove small noise around the image (Figure 2.7D).

\subsubsection{Intenisty of cytosol and cortex}

Actin dynamics mainly happens in the region close to membrane, where the main mechanical support of the cell comes from. Traditionally, this region is called cell cortex and the inner fluidic part is called cytosol. Confocal microscopy takes images from one focal plane of the cell. If the focal plane is in the middle of the cell, then the inner part of the image is the cytosol whereas the outer region is the cortex. On the contrary, if the focal plane is in the bottom of the cell, then only the cell cortex is captured. Therefore, to obtain information from both cytosol and cortex, the focal plane was always carefully choosen to be in the middle of the cell (or at least far from top and bottom of the cell).

To define an optimal region of cortex and cytosol for each cell, the following analysis was always performed: each processed image was first eroded using a disk as a structuring element object. For a given radius of the disk, the total intensity and

${ }^{2}$ If some larger holes escaped the filtering routine, the function imfill(mask,'holes') in Matlab was applied to remove those holes. 

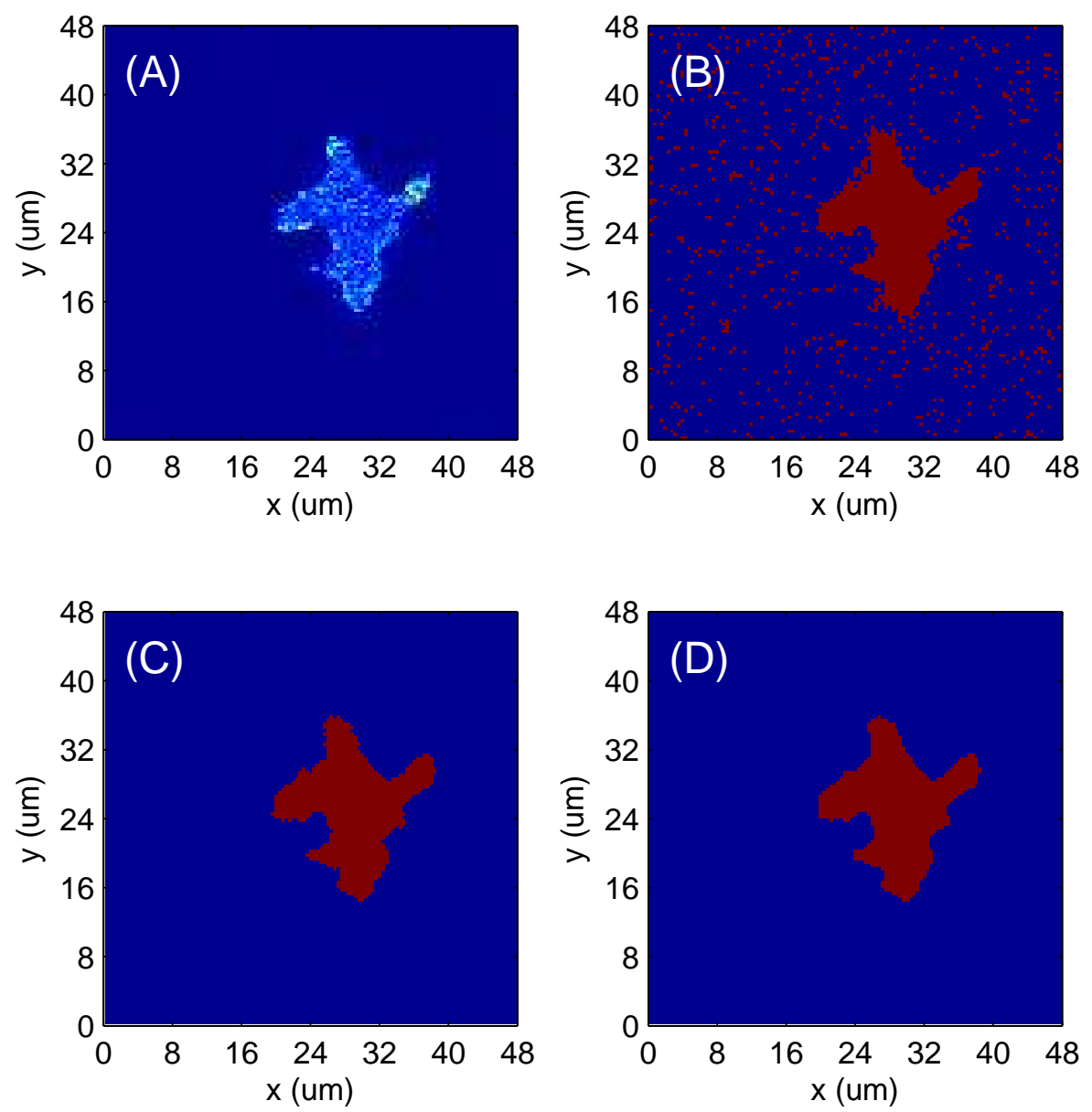

Figure 2.7: Image processing. (A) Original image. (B) Image after removing pixels below the threshold. (C) Image after removing noise with small sized dots $(<1 \mu \mathrm{M})$. (D) Image after removing noise around the edge of the cell (i.e. median filtered image). 
size of the eroded image (i.e., the cortical region of the cell) and the remaining image (i.e., the cytosolic region of the cell) were calculated. The white line in Figure 2.8A shows the boundary of the cell and the yellow line shows the result of an erosion process using a disk with radius of $1.6 \mu \mathrm{m}$. The region inside the yellow line is the cytosol and that between yellow and white line is the cortex. Secondly, this procedure was run at different erosion radii to get the average intensity of the cytosol, the average intensity of the cortex and the size of the whole cell for further area extension analysis.

Figures $2.8 \mathrm{~B}$ and $2.8 \mathrm{C}$ show the average intensity of cytosolic and cortical regions with different size of the erosion disk. The temporal information from different regions of the cell are independent of the size of the erosion region. The intensity of the cytosolic signal converged once no cortical signal was included. In order to quantify the convergence, we calculated:

$$
J=\sum_{t=1}^{N}\left|\left(I_{\text {cytosol }}(\mathrm{r}+1, \mathrm{t})-I_{\text {cytosol }}(\mathrm{r}, \mathrm{t})\right)\right|
$$

$I_{\text {cytosol }}(\mathrm{r}, t)$ is the cytosolic intensity eroded using a disk with radius of $\mathrm{r}$ pixels at frame $t$ and $\mathrm{N}$ is the total number of frames recorded. The cytosolic intensity from the region with minimum value of $\mathrm{J}$ (Figures 2.8D) was then chosen for further analysis.

\subsubsection{Homogeneous cytosolic signal}

Cells usually form localized structures in the cortical region, such as pseudopodia. As only one focal plane is observed with confocal microscope, usually more pixels are included in the cytosolic region (compared to the cortical region) and thus lowers the variability in the extracted structure analysis of cytosolic dynamics. Moreover, the signal of the cytosol is more homogeneous and thus less sensitive to reactions happening in other focal planes. This can be proven by comparing the local intensities in different regions of cytosol and cortex. The image of one cell was cut into 15 radially extending pieces (Figure 2.9, from here on referenced as pizza pieces) and the intensity-time traces of cortex and cytosol from different regions were plotted. In the absence of external stimulation, the signal from cortex and from cytosol varied inside the cell (Figure 2.10A) but the variation among different regions of cytosol was smaller than that of cortex. As one part of our study is to investigate the cellular responses to external stimulation, we next examined the cytosolic and cortical signals under external stimulation. Figure 2.10B shows that the overlaid 

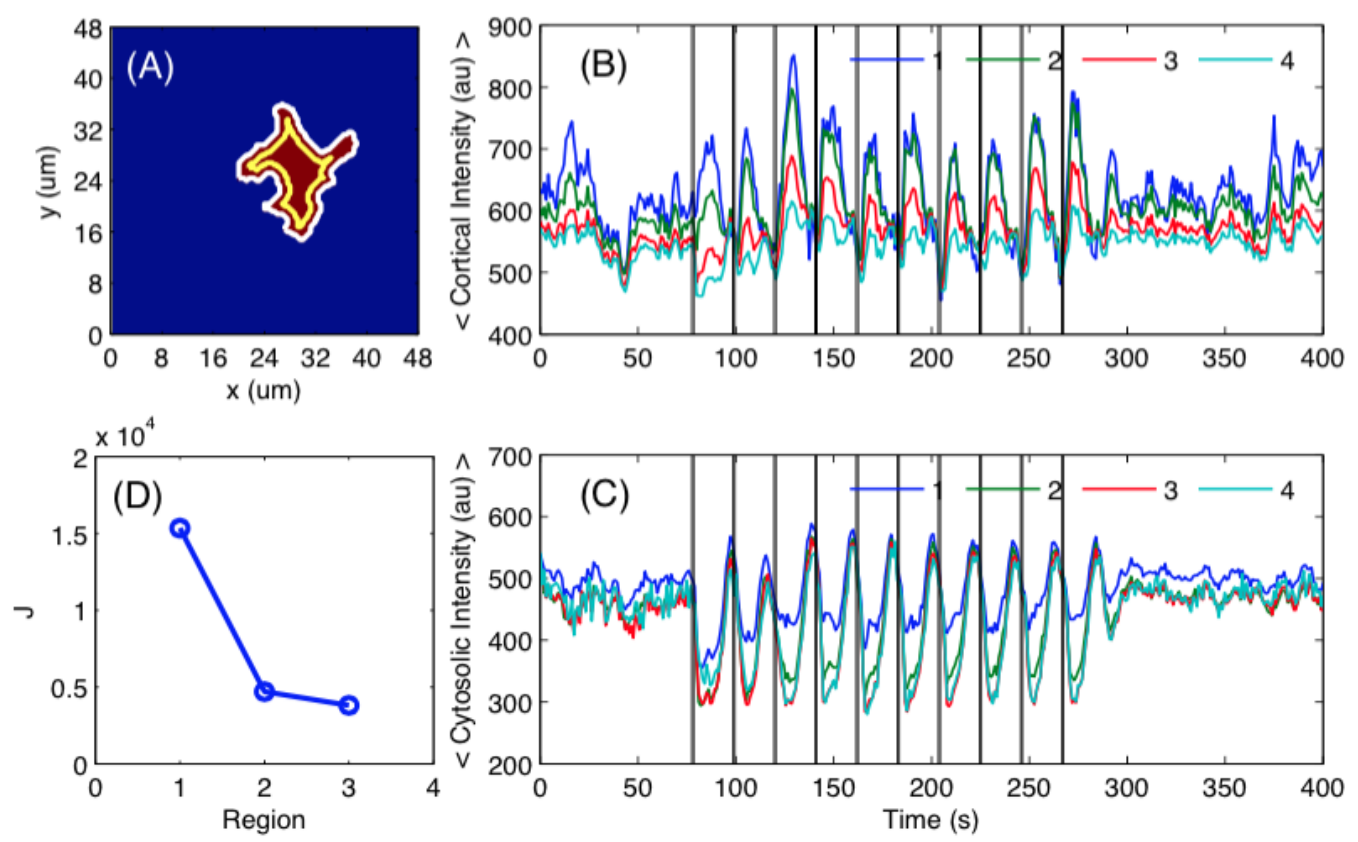

Figure 2.8: Process to obtain optimal cytosolic signal. (A) Each cell was segmented into outer cortex and inner cytosol with optimal size. Segmentation was done by morphological image erosion of the whole cell. Blue refers to the background, red to the entire cell, the white line is the perimeter of the cell, the yellow line is the boundary between cytosol and cortex, as defined for our analysis. (B) Average cortical intensity of varying erosion sizes. Numbers in the legend indicates the radius of the erosion disk with a unit of $0.8 \mu \mathrm{m}$. Different colors show the average signal from the eroded part (i.e., cortical region) with the corresponding erosion sizes labeled in the legend. Vertical black lines show the time points cAMP were applied to the cells. (C) Average cytosolic intensity of varying erosion sizes. Numbers in the legend indicates the radius of the erosion disk with a unit of $0.8 \mu \mathrm{m}$. Different colors shows the average signal from the remaining part after erosion (i.e., cytosolic region) with the corresponding erosion sizes labeled in the legend. Vertical black lines show the time points cAMP were applied to the cells. (D) Sum of the difference between neighboring regions of cytosolic signal in (C). The definition of $J$ is in Eq. 2.1. Region 1 in the $\mathrm{x}$-axis shows the difference of first and second lines (with legend number 2 and 4 respectively). The signal in (C) converges can be seen from the decrease of $J$. 
cytosolic intensities became well aligned with each other once the stimulation started. External stimulation synchronized the actin activity in the entire cytosol and thus made the cytosolic signal perfect for illustrating actin dynamics. Although the variation among cortical signals was reduced by external stimulation, local properties like formation of pseudopod shifted the phases of the local cortical signal. Therefore, describing cortical signal requires a 3D scanning of the whole cell. On the contrary, the homogeneous distribution of cytosolic signals can well reflect dynamics in the whole cell even though the observation is valid for one focal plane only. The fast scan of one focal plane compared to the whole cell also enables us to capture actin dynamics in more detail.

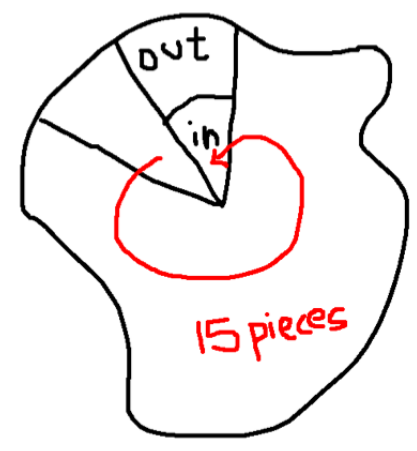

Figure 2.9: Cytosolic signal is better for further data analysis. To test the homogenity of cytosol, one cell was cut into 15 pizza shape slices to compare the intensities of different regions.

\subsubsection{Signal from experiments to data analysis}

For each cell, the optimal cytosol size was obtained from Figure 2.8B. In order to compare the response among various cells and exclude effects such as different expression levels of fluorescent proteins, the cytosolic intensity was normalized to the average intensity before the first application of stimulation. The normalized traces were used for further analysis. The size of the cell was calculated from the number of pixels with a nonzero value in the final processed image (Figure 2.7D). 
(A)
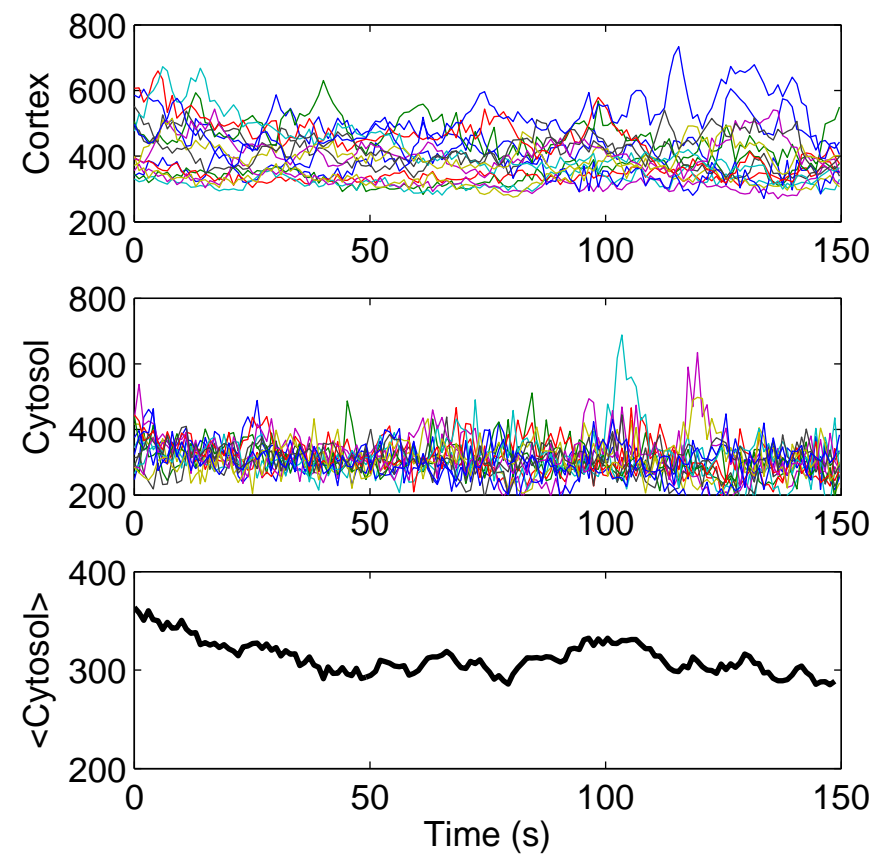

(B)
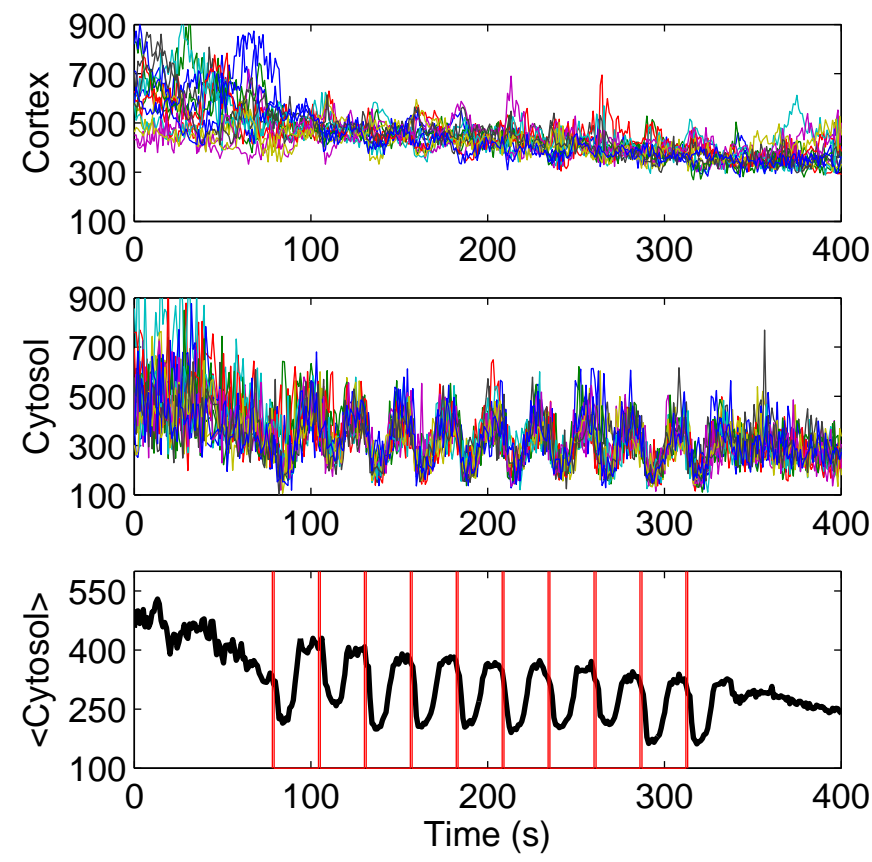

Figure 2.10: Local analysis of fluorescence intensity in different regions inside a cell. Different colors show the average intensity of different pizza slices in the cell (as shown in Figure 2.9). From top to bottom are the intensities of cortex, the corresponding cytosol and the average intensity of the whole cytosol. (A) Traces from one cell in the absence of external stimuli. (B) Traces from one cell periodic stimulated with $1 \mathrm{~s}$ duration and $16 \mathrm{~s}$ intervals. Red lines in the bottom pannel indicate the time points where the stimulation were applied. 


\section{CHAPTER 3}

\section{Self-sustained oscillations}

\subsection{Motivation}

Besides the well known periodic secretion of cAMP [119], it has been reported that Dictyostelium discoideum cells also show non-random oscillations in various other components: the key signaling mediators, Phosphatidylinositol 3,4,5-triphosphate, shape $[99,100]$, locomotion and pseudopodia dynamics in the absence of cAMP [120]. The time period of these oscillations are in the range of serveal minutes. Recently, oscillations of actin cytoskeleton in the range of ten seconds were also found in D. discoideum [109, 110, 117, 121]. As the properties of self-oscillations have not yet been fully investigated, we characterized the oscillation periods, duration and occurrence of self-oscillations. Cells with different passage numbers, starvation times and sizes were also investigated. The aim is to explore the underlying mechanism and understand if there is any role of the self-oscillations in the life cycle of $D$. discoideum cells.

\subsection{Determining self-oscillations}

The occurrence and the disappearance of self-sustained oscillations are unpredictable and the duration of the oscillations is usually short (less than 5 oscillations). Due to the intrinsic noise and intensity variations from cell movement, it is very difficult to determine self-oscillations (Figure 3.1). Here we use an autocorrelation function to remove uncorrelated noise sources and characterize the oscillation periods. If the cytoskeleton shows more than two regular oscillations in the absence of chemoattractant stimulation, we call it self-sustained oscillation or self-oscillation. Considering the fact that the oscillation periods range from $8 \mathrm{~s}$ to $22 \mathrm{~s}[109,110,117,121]$ and 

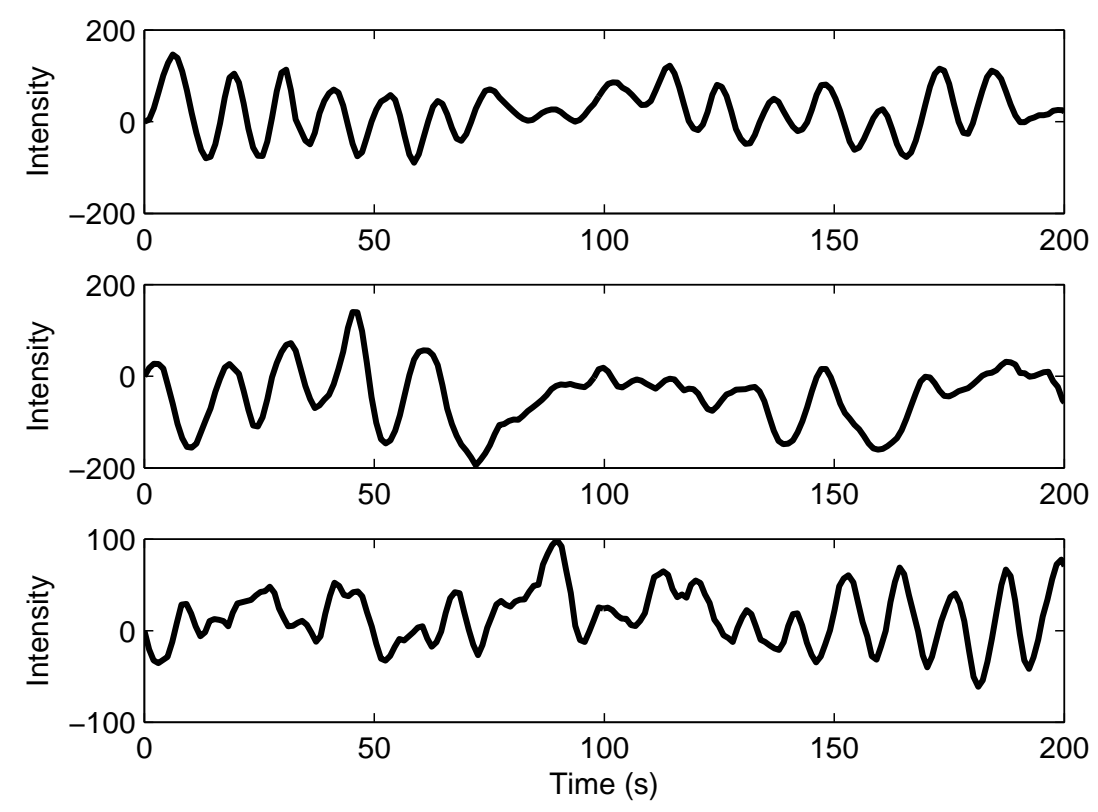

Figure 3.1: Examples of cytosolic intensity of three different cells in the absence of external stimulaion.

the definition of autonomous oscillations requires more than 2 oscillations, a fixed window with a length of $60 \mathrm{~s}$ was used to run autocorrelation. The fixed window was slid across the data to find the autocorrelation at different time. As sudden changes in phase or frequency of the signals affect the analysis of autocorrelation, to run autocorrelation with a fixed length of the window (which is shorter than the whole observation time) not only reduces this effect in the unpredictable signal of self-oscillations but also allows reliable comparison between different cells and different observation times. Figure 3.2A \& 3.2F shows that this method can capture the oscillation periods of regular oscillations (red and green traces) and distinguish the traces without oscillations (blue trace), suggesting that the autocorrelation with fixed-size window can reveal the varying properties of the oscillations.

The autocorrelation function from the fixed window was considered to be a regular oscillation only when it satisfied the following conditions: first, the amplitude difference of neighboring peaks and valleys was larger than 0.02 . Second, the variation of the time differences between peaks (i.e. detected oscillation period) was less than $2 \mathrm{~s}$. Finally, at least two continuous and regular oscillations were detected. 
(A)

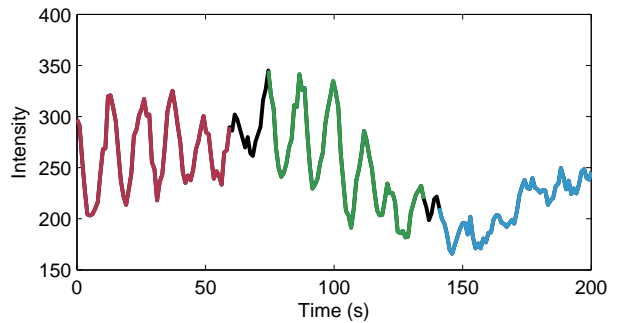

(B)

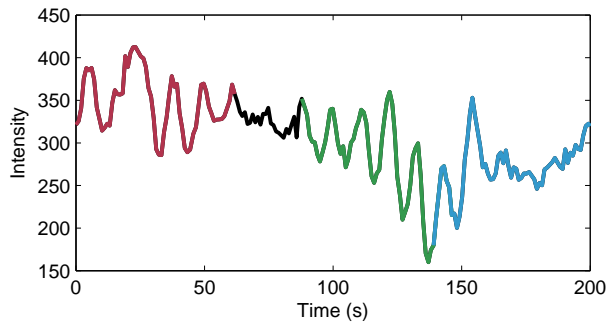

(C)

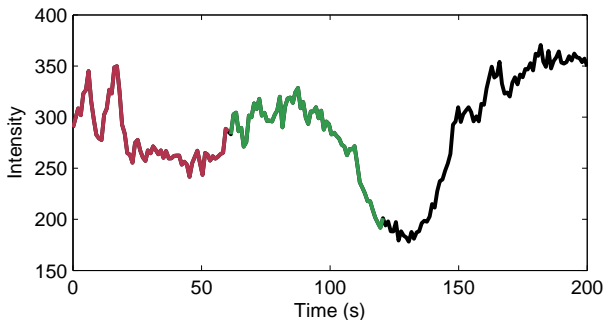

(D)

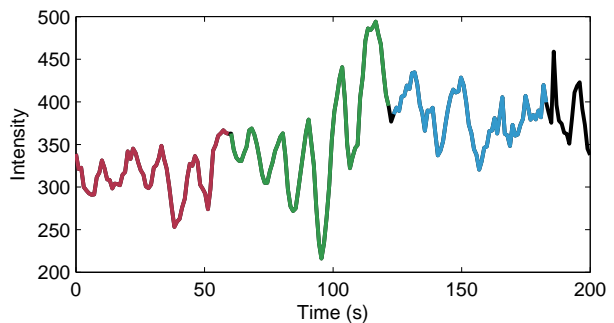

(E)

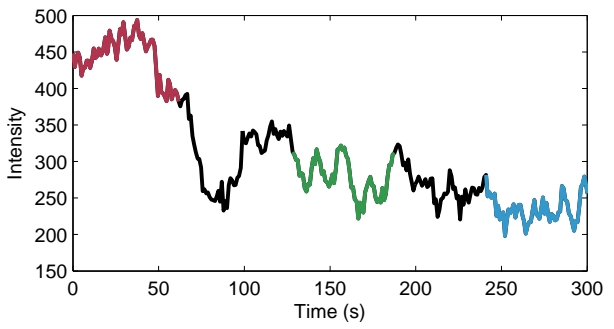

(F)

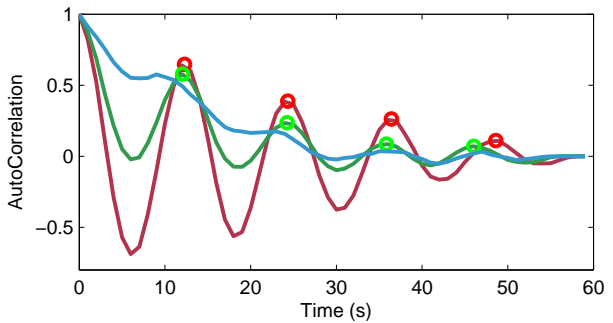

(G)

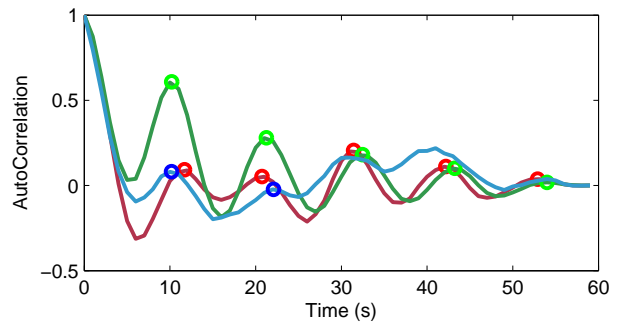

(H)

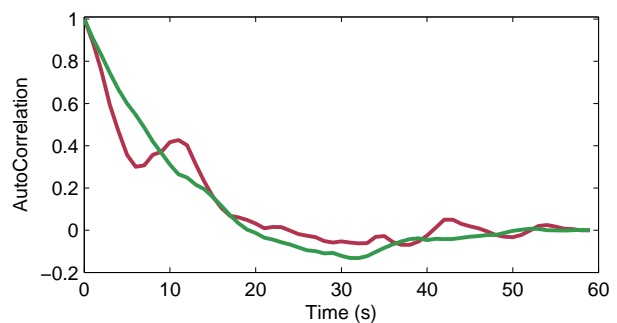

(I)

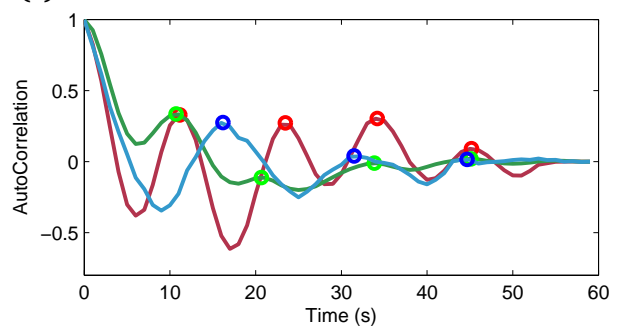

(J)

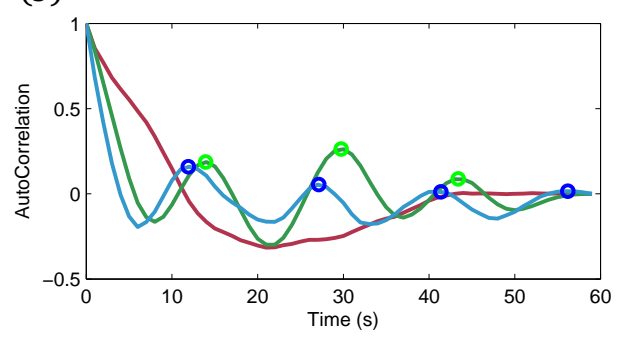

Figure 3.2: Autocorrelation with fixed-size window can reveal the varying properties of the oscilations. (A-E) Examples of different traces of cytosolic signal. (FJ) Autocorrelation analysis. Different colors show the autocorrelation function of the corresponding colored part in the figure of its left hand side. The peaks are marked when the criteria of regular oscillations are met. 

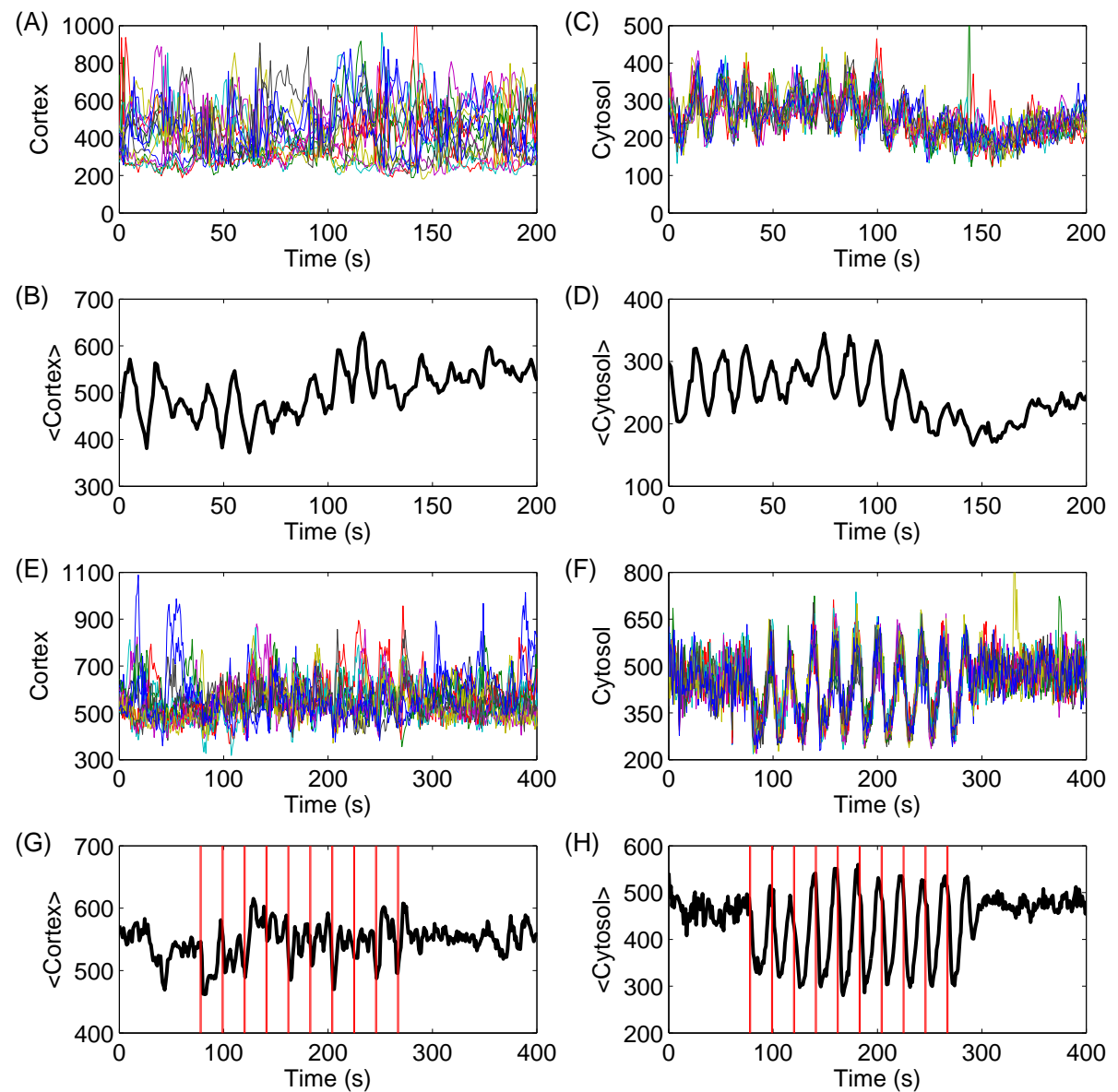

Figure 3.3: Self-oscillating cells are synchronized as cells respond to uniform stimulation. (A)-(D) Intensity analysis of one cell in the absence of external stimulation. (A) Cortical intensity of different regions inside the cell. Different colors show the average intensity of the cortical region in one pizza piece (as described in Figure 2.9). (B) Intensity average over the whole cortex of the cell. (C) Cytosolic intensity of different regions inside the cell. Different colors show the corresponding intensity of the cytosolic region as in (A). (D) Intensity average over the whole cytosol of the cell. (E)-(H) Intensity analysis of one cell in response to uniform and periodic external stimuli (each stimulus lasted $1 \mathrm{~s}$ and the interval between two stimuli was $21 \mathrm{~s}$ ). (E) Cortical intensity of different regions inside the cell. Different colors show the average intensity of the cortical region in one pizza piece. External stimulations were omitted for better visualization. (F) Cytosolic intensity of different regions inside the cell. Different colors show the corresponding intensity of the cytosolic region as in (E). External stimulation were omitted for better visualization. (G) Cortical intensity average over the whole cortex of the cell. Red vertical lines indicate the time when cAMP was applied. (H) Cytosolic intensity average over the whole cytosol of the cell. Red lines indicate the time when cAMP was applied. 


\subsection{Properties of self-oscillations}

We cut the cell into pizza pieces as described in Figure 2.9 to analyze the cytosolic intensity locally. The cytosolic intensity everywhere inside the cell was synchronized in self-oscillating cells (Figure 3.3C) just as the cells respond to uniform stimulation (Figure 3.3F). Cortical signals, on the other hand, varied in different parts of the cell. We used autocorrelation to analyze the cortical and cytosolic signals from different regions individually and found that regular oscillations can only be observed in the cytosolic region. However, the average signal from the whole cortical region showed regular oscillations, implying that the existence of an intrinsic frequency inside the cell comes from the collective behavior of the cortical region. The autocorrelation analysis shows that both the average signal of cytosol and cortex oscillated with a period of $12 \mathrm{~s}$ (Figure 3.4). Moreover, from the cross correlation analysis, we can see that the oscillation of cortex and cytosol were out of phase (Figure 3.4). The homogeneous cytosolic signal can thus perfectly reflect the collective behavior of the cortex to enable us to learn the intrinsic frequency.
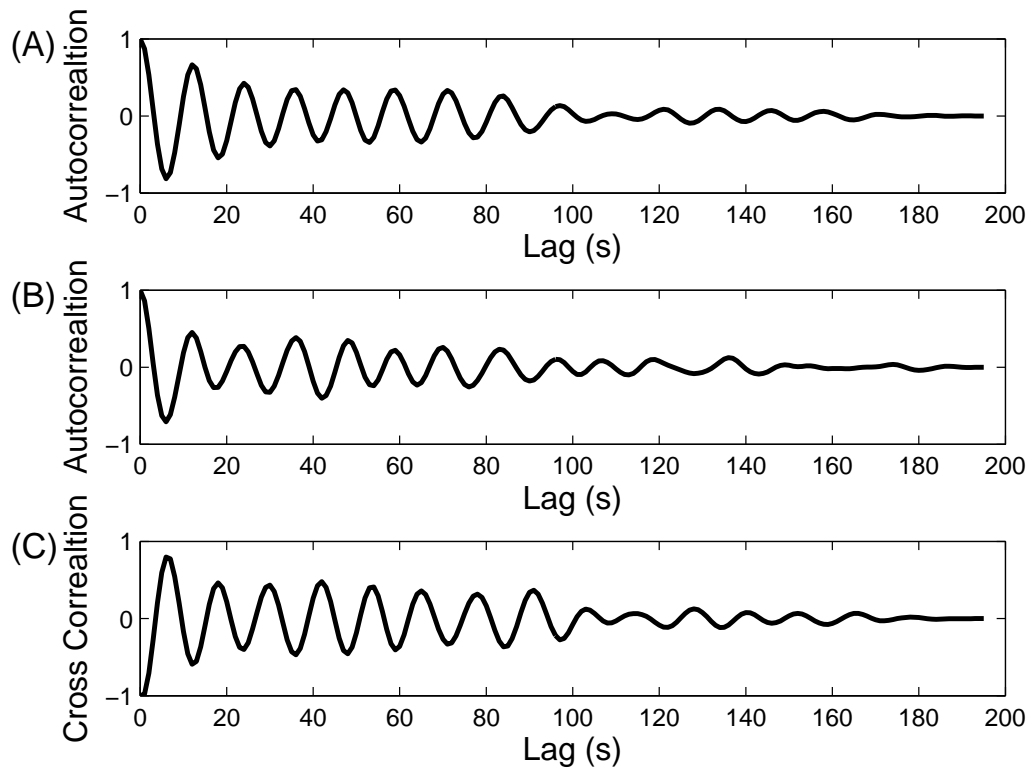

Figure 3.4: Cytosolic signal and cortical signal oscillate out of phase in cells showing selfoscillation. The analysis was based on traces in (B) and (D) of Figure 3.3 . (A) Autocorrelation analysis of the cytosolic signal. (B) Autocorrelation analysis of the cortical signal. (C) Cross correlation analysis of the cortical and cytosolic signal. 


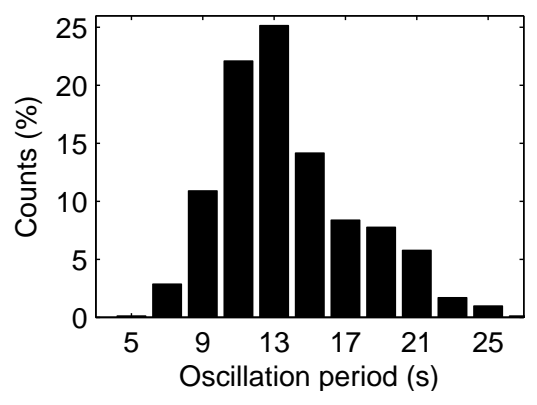

Figure 3.5: Distribution of self-oscillation periods. Fluorescence intensity of cytosolic signal from cells labeled with LimE-GFP were analyzed $(\mathrm{N}=748)$.

To find the distribution of oscillation periods, we observed the LimE-GFP dynamics from wild-type $D$. discoideum cells. $30 \%$ out of 748 cells showed regular oscillations within an observation time of $50 \mathrm{~s}$. The oscillation periods obtained from the peaks of autocorrelation functions were plotted as an histogram in Figure 3.5. Considering that the data acquisition rate was $1 \mathrm{~s}$, the histogram was plotted with a bin size of $2 \mathrm{~s}$ to avoid over interpretation. The distribution of the oscillation periods was $12.7 \pm 4.4 \mathrm{~s}$ (mean \pm s.d.).

To understand how long cells persist to oscillate regularly, we monitored 88 cells in the absence of stimuli continuously for $160 \mathrm{~s}$. We performed autocorrelation analysis with a fixed window size of $60 \mathrm{~s}$ to determine if the trace was oscillating regularly at the given point (Figure 3.6). After converting the cytosolic signal (Figure 3.6A) into traces showing oscillatory on and off states (Figure 3.6D), we collected the longest duration of oscillating-on state from each cell and plotted a cumulated histogram to find the distribution of oscillation durations (Figure 3.7A).

Figure 3.7A shows that during the observation time, more than $50 \%$ of cells oscillated regularly for one third of the measurement time (i.e., $50 \mathrm{~s}, 2-3$ oscillations) but only $19 \%$ of cells persisted for longer than half of observation time (75 s, 6 oscillations). It is worthy to note more than $95 \%$ of cells can at least oscillate around 40 s (i.e., 2 oscillations) during the observation of $160 \mathrm{~s}$, suggesting self-oscillation is a ubiquitous behavior inside all cells. As cells sometimes show self-oscillation but sometimes not, we also analyzed the durations for which the cells stay in oscillatory or non-oscillatory state. The normalized integrated dwell-time histograms for the self-oscillating state and non-oscillating states were fit to an exponential to obtain the average dwell time of both states on the basis of a Poisson distribution. On average, the cells spend longer time in the non-oscillating state than in the oscillating state (Figure 3.7), hinting that self-sustained oscillation could have very well been 

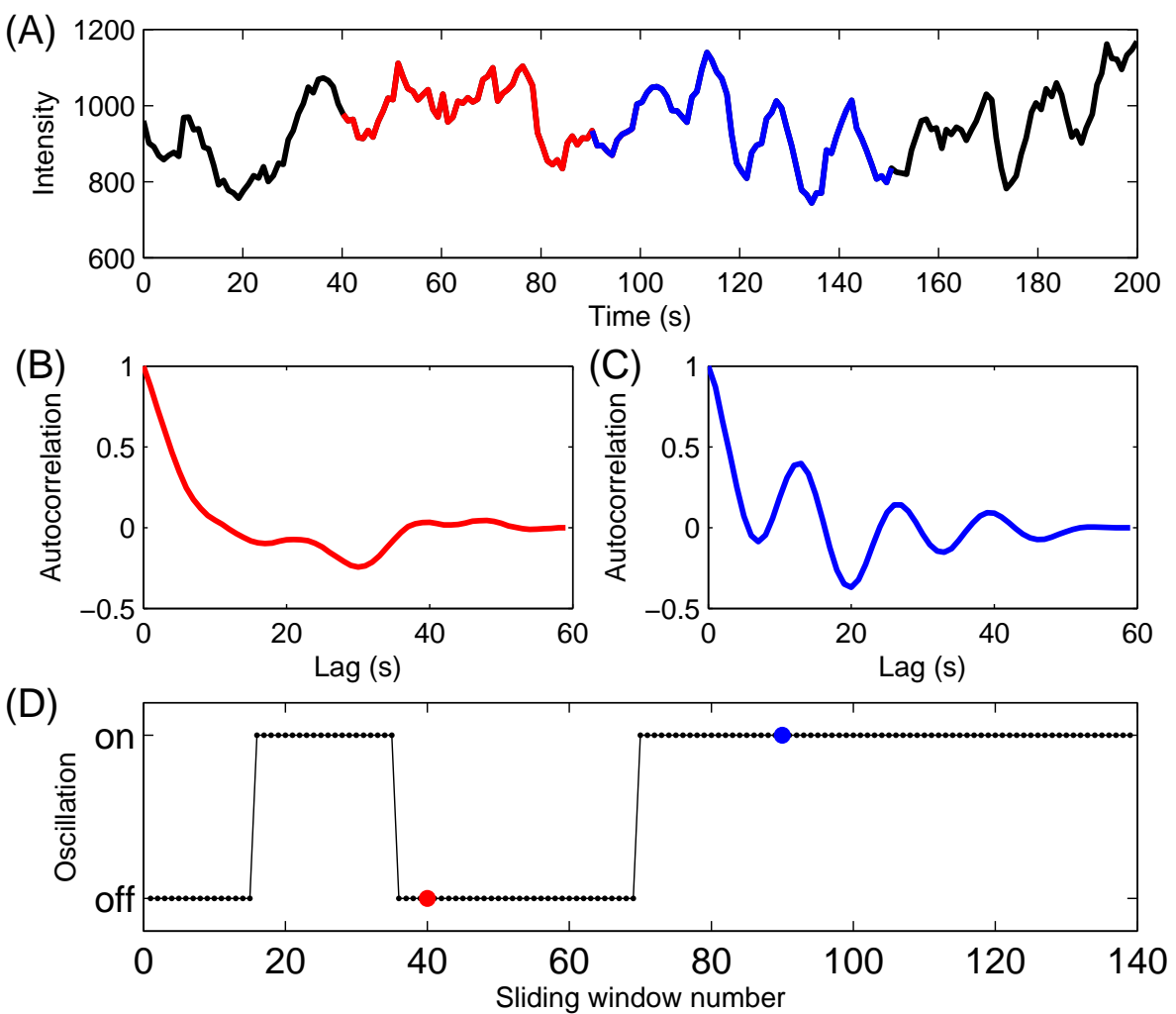

Figure 3.6: Autocorrelation analysis with fixed-size window (60 s) sliding along the whole trace every $1 \mathrm{~s}$ to determine the oscillating and non-oscillating states of cytosolic signal. (A) Cytosolic intensity. (B) Autocorrelation function of the red trace in (A). (C) Autocorrelation function of the blue trace in (A). (D) Oscillatory on and non-oscillatory states. Red and blue points are the analyzing windows of the red and blue traces respectively. 
overlooked if the cells had not been observed without external stimuli for an extended period of time.
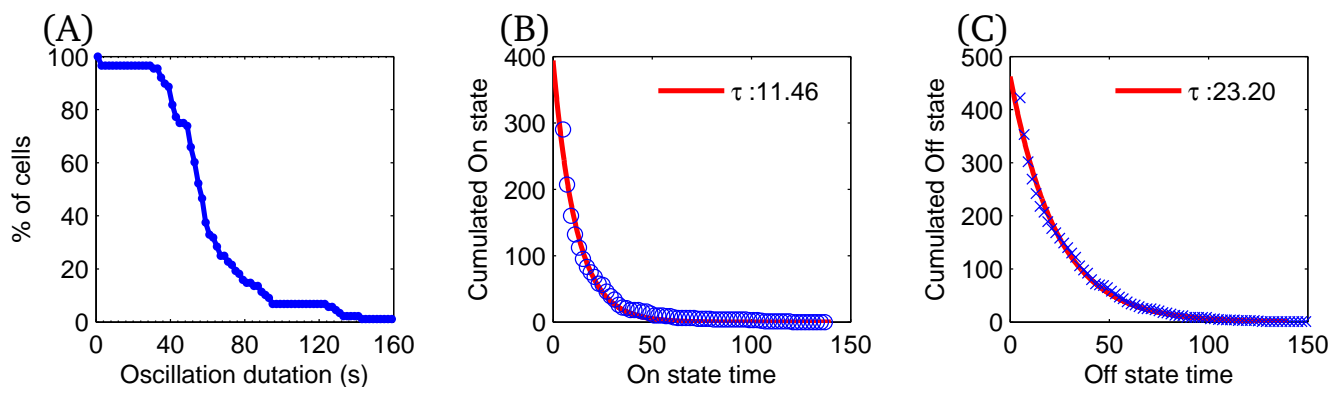

Figure 3.7: Analysis of oscillation dwell time. 88 wild-type cells labeled with LimE-GFP were observed for $160 \mathrm{~s}$ in the absence of external stimulation. (A) Distribution of the longest time one cell can oscillation during the observation. (B)\&(C) Distribution and the average lifetime of the oscillatory and non-oscillatory states. Circles are experimental data and the red line is the fit to a single exponential decay $\left(y=a \exp ^{-b x}, \tau=1 / b\right)$. The time of on and off states was counted as the number of sliding windows described in Figure 3.6.

\subsection{Impact of various cell properties on self-oscillations}

Although it has been known that self-sustained oscillations can be observed in many proteins that regulate actin filament dynamics and can occur independent of the signaling network [110], the detailed mechanism is still not clear. Since cell-to-cell variability exists even within the same cell strain, we examined factors like passage number in detail to verify if the self-sustained oscillations came from genetic instability accumulated due to long-term subculture. Figure 3.8A showed no difference in the oscillation period distribution at different passage numbers. This implies that subculturing does not accumulate genetic instability relevant to actin dynamics (at least unnoticeable till passage number 13).

As $D$. discoideum cells enter a developmental cycle upon food deprivation, distinctive characters might be present at different starvation times. We next inspected the effect of starvation time on self-sustained oscillations. Figure 3.8B shows that the distribution of oscillation period was independent of the starvation time.

We next investigate if the size of the cell characterizes the oscillatory state. The histogram of the oscillation period were comparable for various sizes of wild-type cells (Figure 3.8C). 
(A)

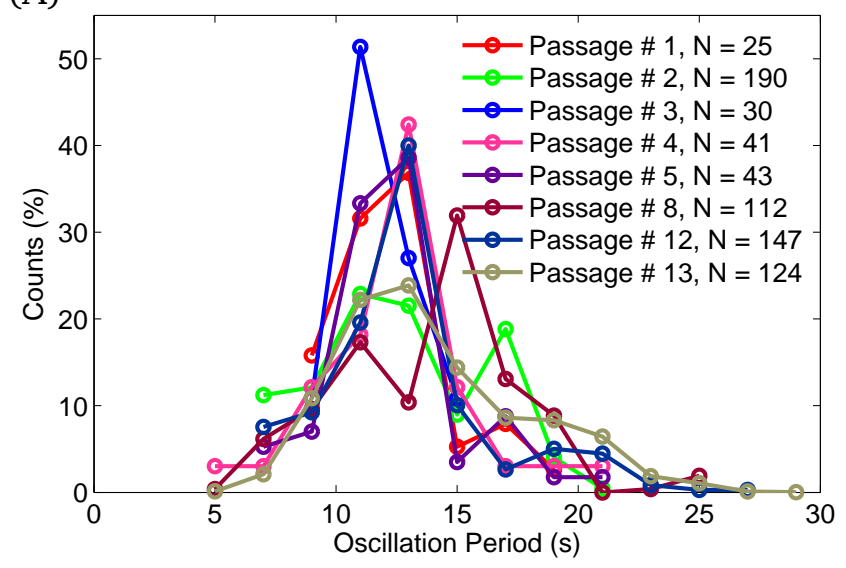

(B)

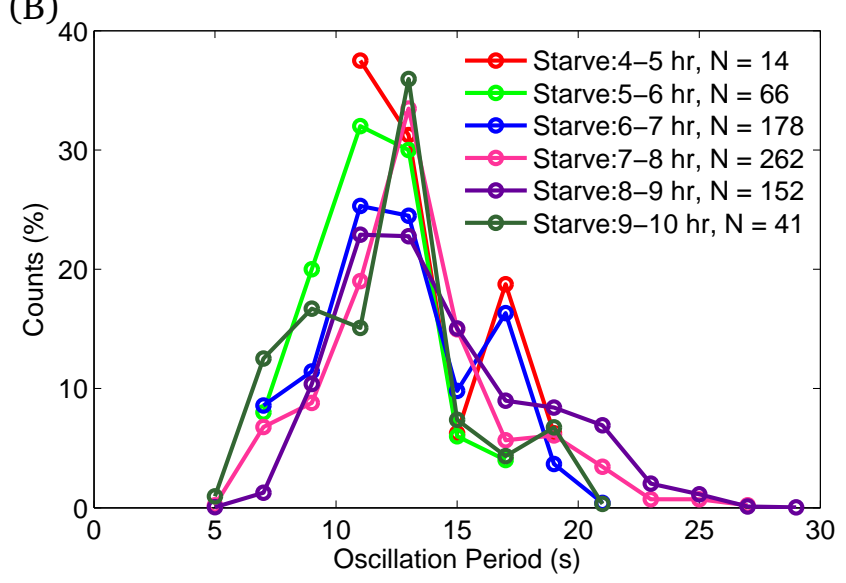

(C)

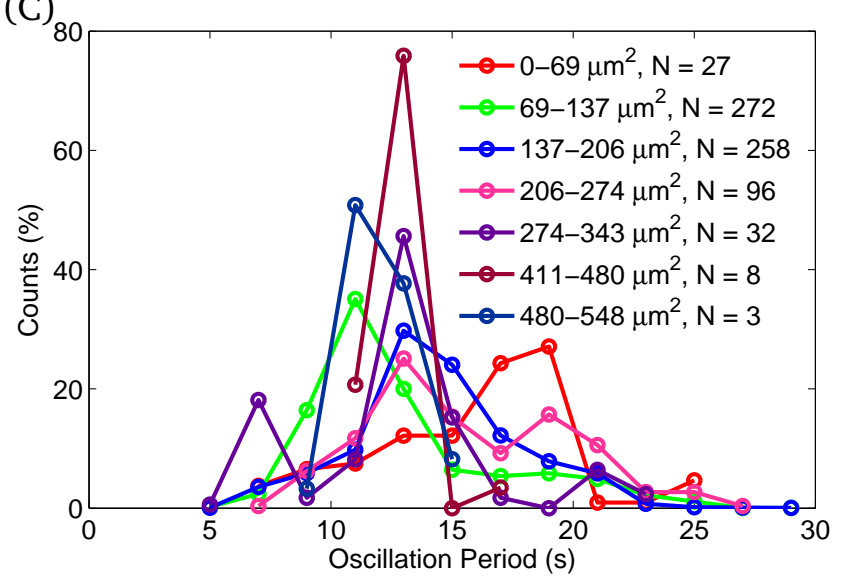

Figure 3.8: Impact of different passage numbers, starvation times and cell sizes in the distribution of self-oscillations. Legend shows the number of cells under different condition. (A) Distribution of oscillation period for self-oscillating cells with different passage numbers, (B) different starvation times and (C) with different cell sizes. 


\subsection{Proposed underlying mechanism}

It has been reported that mast cells showed actin oscillations with a period of around $30 \mathrm{~s}$. This oscillation was found to be regulated by calcium oscillations coupled to $\mathrm{PI}(4,5) \mathrm{P}_{2}$ oscillations [122]. Oscillations of calcium [123-125] and $\mathrm{PI}(4,5) \mathrm{P}_{2}$ [99, $100]$ have also been reported in $D$. discoideum cells but with a much longer oscillation period (longer than $3 \mathrm{~min}$ ). As the oscillation signal from the upstream cAMP signaling components is much slower than the observed self-oscillations, it is unlikely that self-oscillations of LimE are regulated by upstream signals like the calcium or $\mathrm{PI}(4,5) \mathrm{P}_{2}$ oscillations.

As the actin network itself can continuously polymerize and depolymerize by varying the interactions between actin and other regulatory proteins, another possible origin of self-oscillations is the self-organization of actin network. In this case, similar oscillations should be also observed in actin regulatory proteins. The fact that selfoscillations have been reported in proteins that are involved in actin treadmilling like the components of SCAR/WAVE and Coronin with oscillation period similar to LimE $[110,117,126]$ supports this hypothesis.
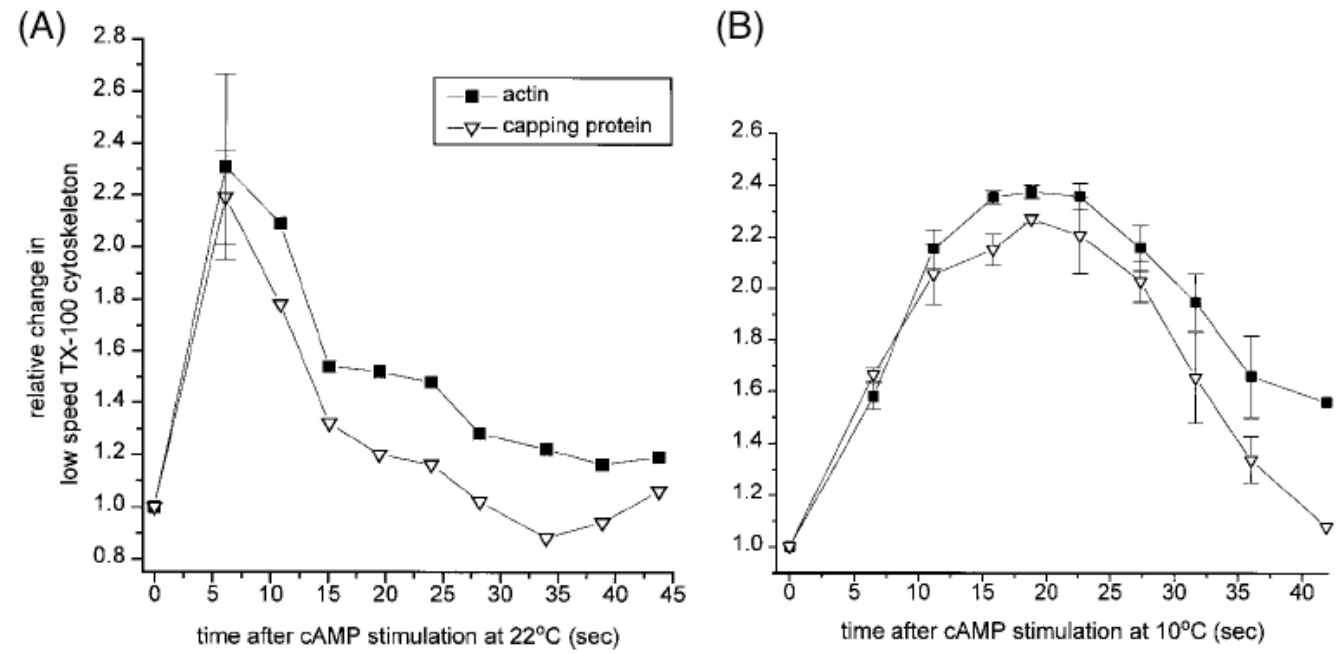

Figure 3.9: Capping protein and actin peaked in the cortical region at the same time. Figures were adapted from the observations of Eddy and Condeelis [127]. At different times after stimulation with $10 \mu M$ cAMP, the amount of actin (square) and capping protein (triangle) at the corresponding time were determined by Western blot. (A) D. discoideum AX3 cells were starved for $5.5 \mathrm{~h}$ at $22^{\circ} \mathrm{C}$ and then treated with $3 \mathrm{mM}$ caffeine for $30 \mathrm{~min}$. (B) D. discoideum AX3 cells were starved for $5.5 \mathrm{~h}$ at $22^{\circ} \mathrm{C}$ but then transferred to $10^{\circ} \mathrm{C}$ for $30 \mathrm{~min}$ in the presence of $3 \mathrm{mM}$ caffeine. 
Here we tried to suggest the involvement of capping protein. Capping protein is essential for terminating actin polymerization [35, 36]. Condeelis and his colleagues [127] have observed that capping protein is usually distributed in the cytosol; however, in response to stimulation by cAMP, not only did F-actin grow in the cortex but also the capping proteins translocate from the cytosol to cortex. The amount of capping protein peaked at the same time as F-actin after the stimulation by cAMP and then decreased together (Figure 3.9). Since we do not have cells with capping proteins labeled, we regarded the time that cortical actin filaments reach maximum as the binding time of capping protein. Figure 3.10A) shows the time at which the cytosolic LimE-GFP signal reached a minimum after cells respond to cAMP (i.e., cortical filamentous actin reaches maximum, $T_{p}$ ). As an oscillation is composed of polymerization followed by depolymerization (Figure 3.11A), we can also analyze the polymerization time of self-oscillating cells $\left(\mathrm{SO}_{\mathrm{Tp}}\right.$ in Figure 3.11A). The similar distribution of capping protein binding time (Figure 3.10B) and the polymerization time of self-oscillating cells $\left(\mathrm{SO}_{\mathrm{Tp}}\right.$ ) suggests that it is the binding of capping protein that terminates the polymerization of self-oscillations.
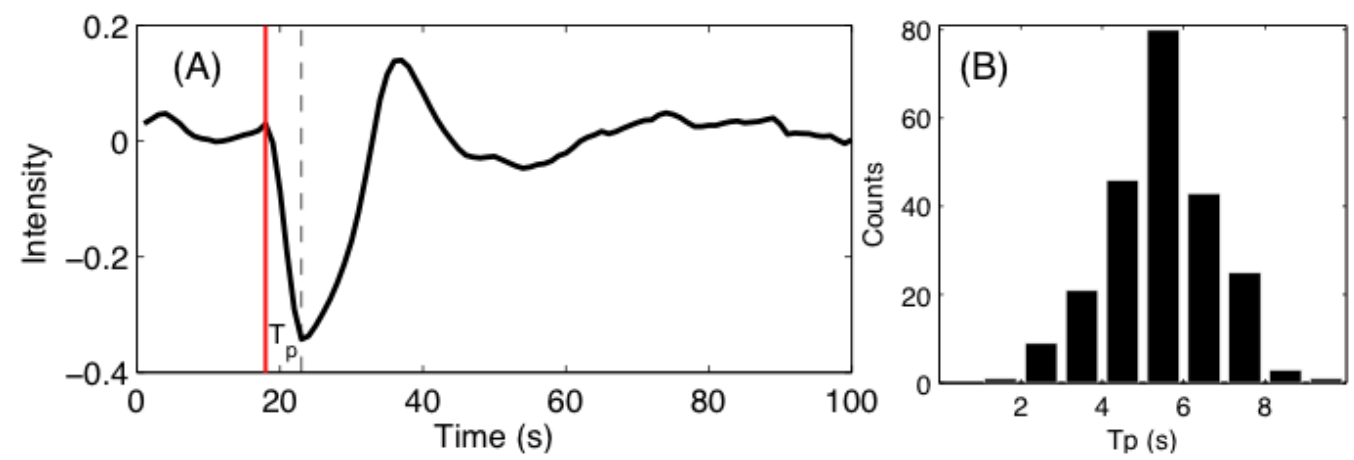

Figure 3.10: Learn the time of capping protein binding from the response to cAMP. (A) A typical response of LimE-GFP in wild-type cell to $1 \mathrm{~s}$ stimulation. Red line indicated the time cAMP was applied to the cell. (B) Distribution of polymerization time $\left(T_{p}\right) . T_{p}$ was defined by the time between stimulation applied and the time cytosolic signal reached minimum (vertical dashed line). 124 wild-type cells labeled with LimE-GFP responded to one $1 \mathrm{~s}$ stimulation were observed.

The amplitude of self-oscillation reflects the amount of actin filaments formed. As actin filaments grow mainly from the barbed end of the filament, more branches in actin network allows more actin binding to the actin filaments at one time. Since the binding of capping proteins terminates the elongation of actin filaments, both the binding time of capping protein $\left(\mathrm{SO}_{\mathrm{Tp}}\right)$ and the extent of actin branches can affect the amount of formed actin filaments. Figure 3.11B shows that the amplitude 
of self-oscillation is not related to the amount of Arp2/3, the main protein that enabling branches formation. Considering the fact that SCAR/WAVE, the main protein that activates Arp2/3, is activated through external stimulation [128-131], it is possible that only few active Arp2/3 in cells in the absence of external stimuli and thus shows no effect on the formation of actin filaments. This is confirmed by comparing the amount of Arp2/3 translocate to the cortical region in the presence and absence of stimulation (Figure 3.12). On the other hand, the binding time of capping proteins controls the amount of actin formation: the later the binding of capping proteins occurs (larger $\mathrm{SO}_{\mathrm{Tp}}$ ), the longer the actin filaments can grow (larger $\mathrm{P}_{\text {Amp }}$, Figure 3.11B). Due to the fact that depolymerization can only initiate from the pointed end of actin filament, longer filaments requires longer time to depolymerize, as seen by Figure 3.11E. As an oscillation is composed of polymerization followed by depolymerization (Figure 3.11A), it is the binding time of capping protein that determines the frequency of self-oscillation.

It is worthy to note that the distribution of polymerization $\left(\mathrm{SO}_{\mathrm{Tp}}\right)$ and depolymerization times $\left(\mathrm{SO}_{\mathrm{Td}}\right)$ are indistinguishable (D and $\mathrm{F}$ in Figure 3.11), suggesting that the traces of self-oscillation were very symmetric.

\subsection{Pseudopod formation and self-oscillations}

Different time scales have been observed in $D$. discoideum before: adenylate cyclase produces cAMP every 6 min, phosphatase and tensin homolog (PTEN) periodically oscillate out of phase with phosphoinositide-3-kinase (PI3K) with a period around $200 \mathrm{~s}$. Pseudopod grows with a period of around $12 \mathrm{~s}$ [101]. The recent observations of rapid oscillations in the cytoskeleton network have a period around $10 \mathrm{~s}[109,110$, 117]. Although the time scale of this rapid oscillation is close to that of pseudopod growth, the link between self-oscillations and pseudopod formation is still unclear.

Based on previous studies, we know that the growth period of a pseudopod [101] is comparable with the period of self-oscillation. Secondly, Van Haastert and his colleagues found that $D$. discoideum cells formed pseudopod with the same frequency at different starvation times [132]. Thirdly, cells lacking myosin II, moved less efficiently toward cAMP because myosin II is essential for inhibiting lateral protrusions to polarize the cell and enables persistent directional movement. However, cells were still able to extend pseudopod or ruffled membranes even without myosin II [133136]. 

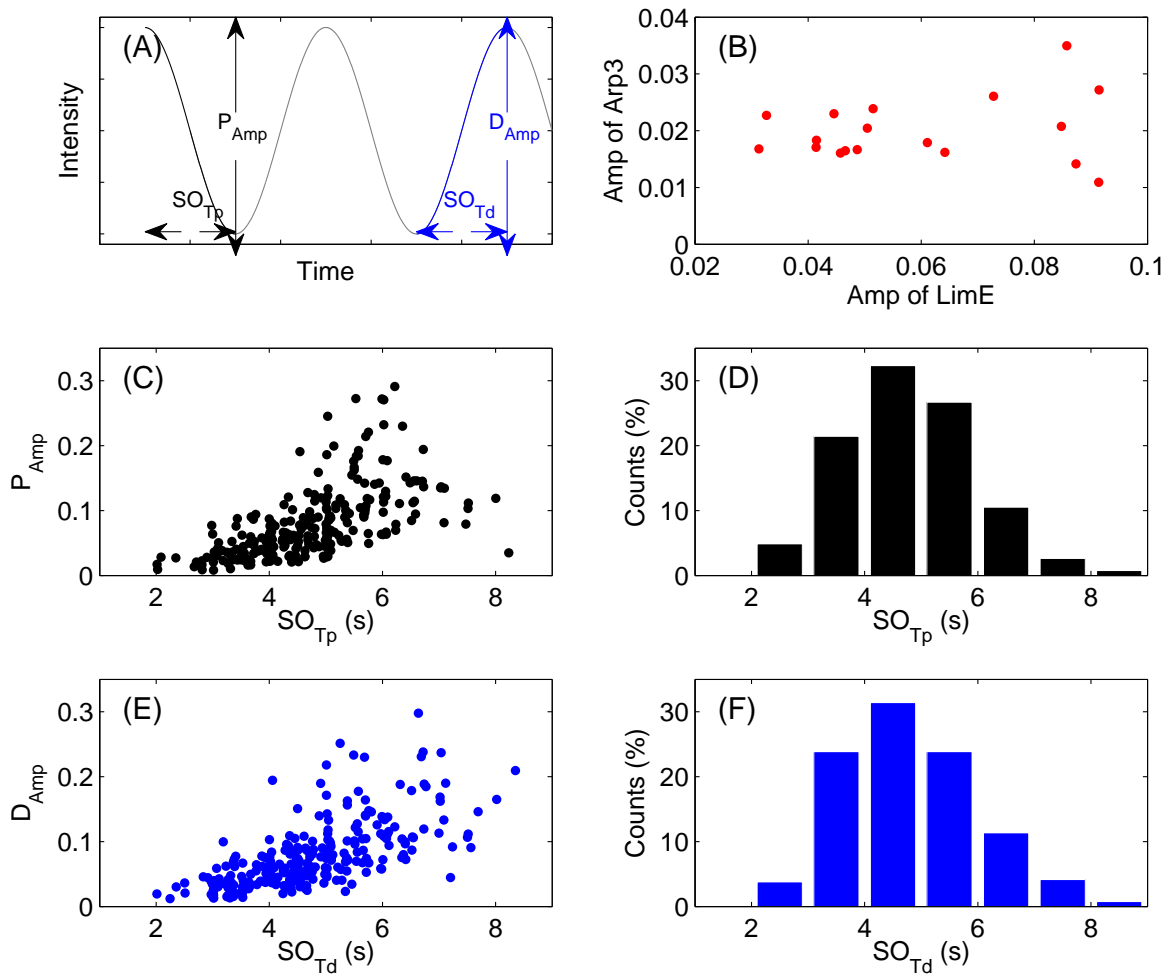

Figure 3.11: Self-oscillating signals show symmetric oscillations. 266 self-oscillating wildtype cells labeled with LimE-GFP were observed in the absence of external stimulation. SO stands for self-oscillation. (A) Definition of polymerization time $\left(\mathrm{SO}_{\mathrm{Tp}}\right)$, polymerization amplitude $\left(\mathrm{P}_{\mathrm{Amp}}\right)$, depolymerization time $\left(\mathrm{SO}_{\mathrm{Td}}\right)$ and depolymerization amplitude $\left(\mathrm{D}_{\mathrm{Amp}}\right)$. (B) Amp from self-oscillating cells co-express Arp3-GFP and LimE-mRFP. Each red dot shows the analysis from one self-oscillating cell. (C) $\mathrm{SO}_{\mathrm{Tp}}$ v.s. $\mathrm{P}_{\mathrm{Amp}}$. Each dot shows the average $\mathrm{SO}_{\mathrm{Tp}}$ and $\mathrm{P}_{\mathrm{Amp}}$ from one cell. (D) The distribution of $\mathrm{SO}_{\mathrm{Tp}}$. (E) $\mathrm{SO}_{\mathrm{Td}}$ v.s. $\mathrm{D}_{\mathrm{Amp}}$. Each dot shows the average $\mathrm{SO}_{\mathrm{Td}}$ and $\mathrm{D}_{\mathrm{Amp}}$ from one cell. (F) The distribution of $\mathrm{SO}_{\mathrm{Td}}$. 


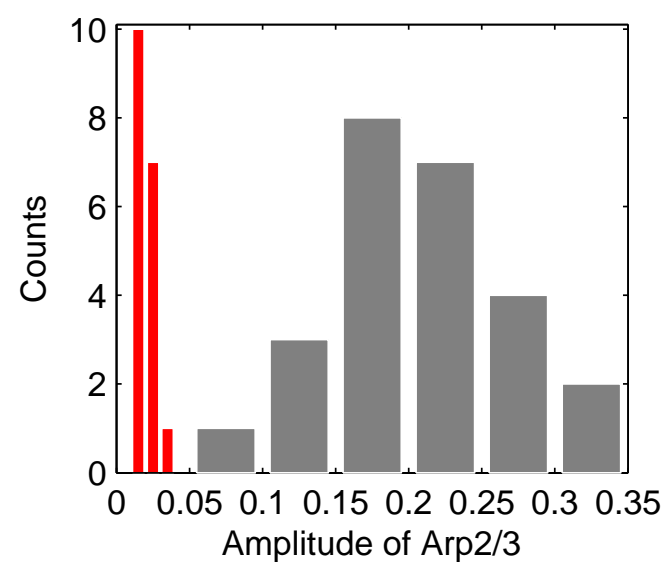

Figure 3.12: Arp2/3 is activated by external stimulation. Histogram of the amount of Arp $2 / 3$ translocate to the cortical region in the absence (red, $\mathrm{N}=18$ ) and presence (gray, $\mathrm{N}=25$ ) of external stimulation.

Comparing the properties of self-oscillations we found in this study with what is already known about pseudopod from literatures, we find it likely that the selfoscillation controls the global time scale governing the local properties of pseudopod. We next looked at how cells changed their morphology while self-oscillating. Figure 3.13A shows the cytosolic signal of one self-oscillating cell. Yellow bars mark the decreasing part of cytosolic signal. Cell shapes of the corresponding numbers are plotted in Figure 3.13B. The red regions are the parts where the cell extended during the decrease of cytosolic signal. The black regions are the parts where the cell contracted and gray regions are the parts without change. The decrease of the cytosolic signal is either related to the extending pseudopod (1-4 of Figure 3.13B) or just the undulation of the cell membrane (5-8 of Figure 3.13B).

Oscillations of actin dynamics reflect either the protrusion of pseudopod or random undulations of the plasma membrane. As actin dynamics drive cell movement by applying forces to the membrane, more actin filaments formed in the cortical region (i.e., bigger $\mathrm{D}_{\mathrm{Amp}}$ of cytosolic signal) might lead to a stronger force. We therefore analyzed the area extended in the cells during every oscillation. Figure 3.14 shows how membrane protrusion relates to the decrease of cytosolic LimE-GFP intensity. In general, cells extended a larger area when they polymerized more actin (Figure 3.14A) but the extension rate was comparable for different amounts of actin formation (Figure 3.14B). Bosgraaf and Van Haastert also observed that different pseudopods always move with a constant speed [101]. This observation implies that in cells where actin hits the membrane with larger strength more area 


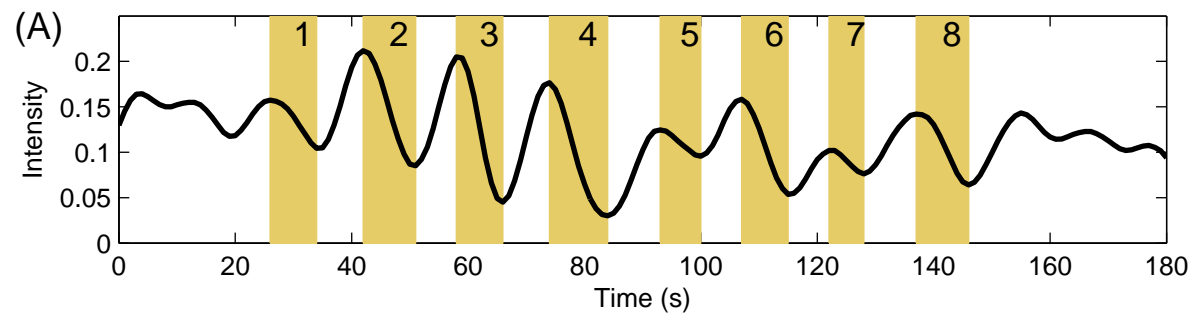

(B)
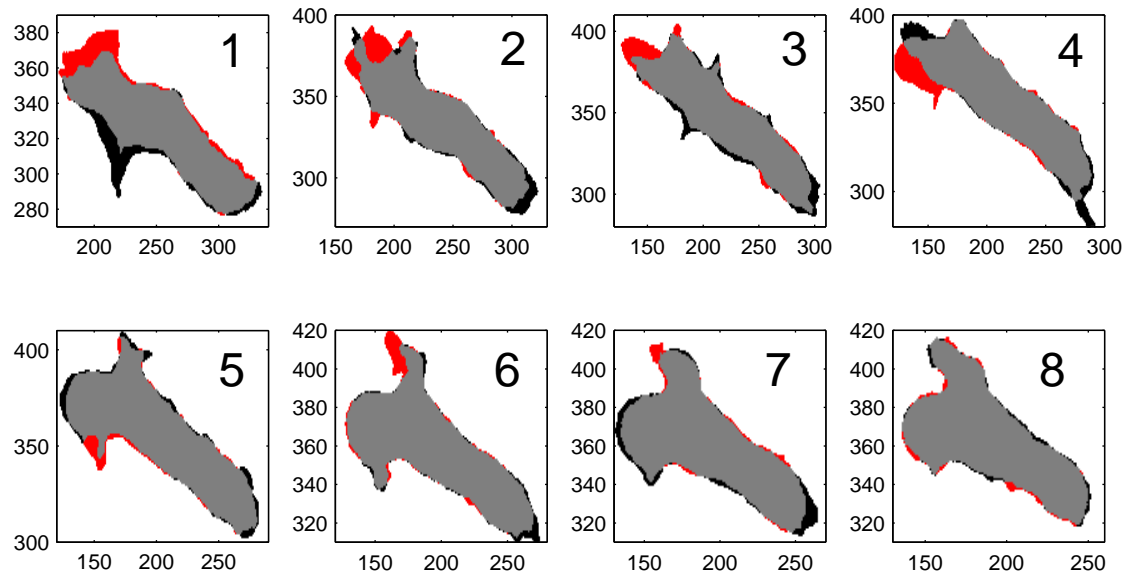

Figure 3.13: Self-oscillations in the absence of external cAMP was related to the membrane undulation of the cells. (A) Cytosolic signal of LimE-GFP in one self-oscillating wild-type cell. (B) Cell shapes varied with the actin signal. Each box shows the cell contour of the yellow region of the corresponding number in (A). Comparing the cell contours at the beginning and end of the yellow region of the corresponding number, red shows the extended part of the cell, black shows the contracted part and gray shows the unchanged part. $\mathrm{X}$ and $\mathrm{y}$ axes show the position of the cell with a unit of pixel ( 1 pixel $=0.414 \mu \mathrm{m})$. 
is protruded but then actin hits the membrane less often. The frequency of hitting the membrane was controlled by the fact that it takes longer to depolymerize more actin filament (Figure 3.11D). Therefore, by simply manipulating the polymerization and depolymerization, the self-organization of the actin network might result in a constant extension rate of pseudopod to explore the surrounding for food during starvation.
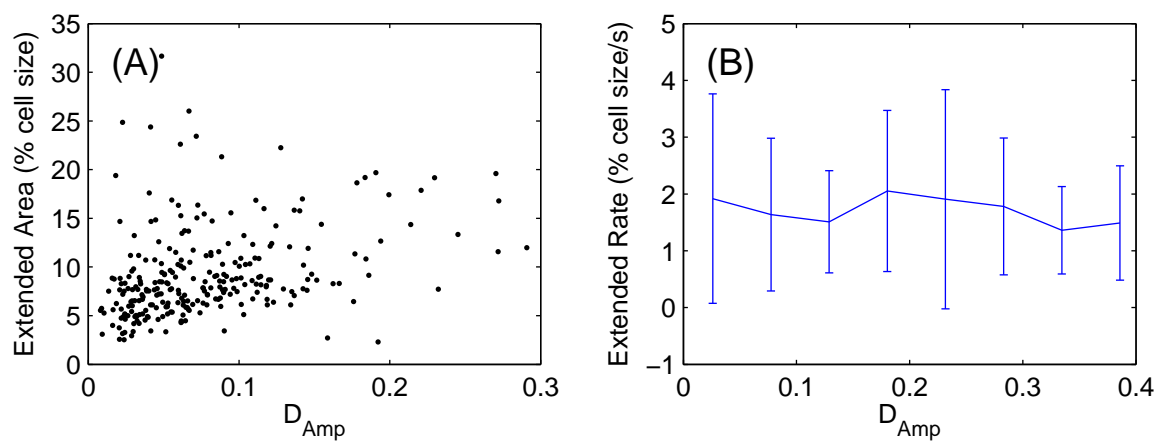

Figure 3.14: $D$. discoideum cells show constant protrusion rate. 266 self-oscillating wildtype cells labeled with LimE-GFP were observed in the absence of external stimulation. (A) Extended area during the depolymerization time of one oscillation was plotted against the corresponding depolymerization amplitude $\left(\mathrm{D}_{\mathrm{Amp}}\right)$. (B) Extension rates at different $\mathrm{D}_{\mathrm{Amp}}$. Extension rates were calculated by dividing depolymerization time $\left(\mathrm{SO}_{\mathrm{Td}}\right.$ in Figure 3.11) into the corresponding extended area (Extended area in (A)).

However, by looking at only one focal plane, we lost the three dimensional information of pseudopods. The correlation between area and response amplitude (Figure 3.14A) was thus not as obvious as the amplitude-time relation observed in Figure 3.11D. A clearer trend might be found in experiments that can better capture pseudopod dynamics, like scanning the whole cell faster in 3D or using microfluidic techniques to flatten cells into a two dimensional plane [137]. 


\subsection{Conclusion}

In this study, we examined the properties of self-oscillations: by systematically examining the oscillation period of more than 700 cells, we first confirmed that the distribution of the self-oscillation period peaks around $12 \mathrm{~s}$ (Figure 3.5). As asynchronous signals average each other out, we can describe the properties of the oscillations only when the whole actin network is synchronized. This intrinsic frequency governing the actin dynamics and its property is robust; cells with different passage numbers, starvation times and different sizes oscillate with similar periods. This oscillation relates either to the local protrusions of pseudopods for directional movement or to the formation of patches for membrane undulations. By considering the roles of proteins regulating the actin network, we concluded that (i) the frequency of self-oscillations are determined by the binding time of capping proteins, (ii) the binding time of capping proteins determines the amplitude of self-oscillations, and (iii) the amplitude controls the following depolymerization time.

Considering the facts that oscillatory and non-oscillatory states alternated inside every cell and that the oscillation amplitude and frequency vary with time, it is possible that the self-oscillation is just a consequence of random synchronization of many local oscillators inside a cell. It has been reported that oscillations at different spots (local oscillators) within the same cell could oscillate with a phase shift or different frequencies [110]. This observation is consistent with Figure 3.3B, which shows that self-oscillations originate from the collective behavior of the whole actin network. Taken together, the self-oscillations just reflect the synchronization of rapid turnover in the self-organized cytoskeletal network. 


\section{CHAPTER 4}

\section{Actin dynamics is stimulation strength dependent}

\subsection{Motivation}

In the previous chapter, we learned the dynamics of filamentous actin in the absence of external stimulation. Careful analysis showed that the intrinsic oscillation period centered around $12.7 \mathrm{~s}$ with a standard deviation of about $4.5 \mathrm{~s}$ (Figure 3.5 ). The oscillations show the same time scale as the rapid oscillations of cytoskeletal network observed in [110]. Westendorf et al., found a resonance peak at $20 \mathrm{~s}$ when they stimulated the cells periodically [109]. The resonance period is close but does not coincide with the intrinsic oscillation period of self-oscillations. It is still unknown if this difference is significant. Is this a reasonable error based on cell-to-cell variability or are there other controlling mechanisms?

The key to link the two time scales relies on experiments that can observe the transition of actin dynamics from self-oscillation to controlled cAMP response. $D$. discoideum cells were starved to become chemotatic so that cAMP can be used as chemoattractant. To have precise control of the application of cAMP, we combined photolysis with microfluidics: a caged cAMP compound was continuously flowed into the microfluidic channel, as described in chapter 3 . We visualized the dynamical behavior of the actin cytoskeleton by expressing fluorescently tagged LimE $\Delta$ coil (LimE-GFP), which is known to co-localize with freshly polymerized actin filaments $[80,138]$. By comparing the responses before and after the application of stimuli, we learn not only how the external stimuli change the self-oscillations but also how self-oscillations affect the responses to cAMP stimulation. 

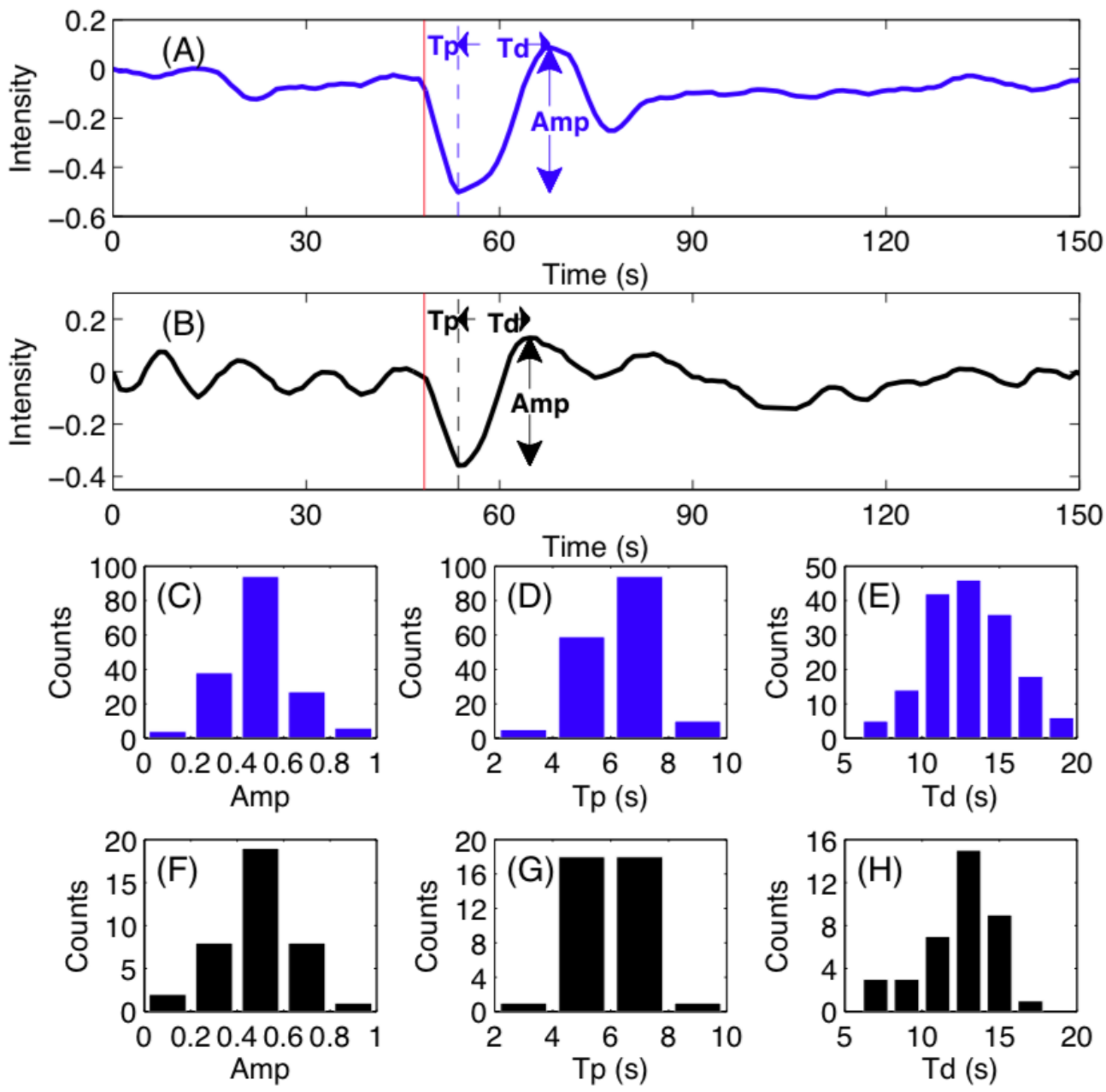

Figure 4.1: Effects of self-oscillations on actin dynamics in response to a single $1 \mathrm{~s}$ stimulation. LimE-GFP signal from wild-type cells were observed. Vertical red line marks the time of cAMP application. $\mathrm{T}_{\mathrm{p}}$ was defined as the time between the stimulation applied (i.e., vertical red lines) and the intensity reached minimum (i.e., vertical dashed lines). $T_{d}$ was defined as the time between the minimum (valley, also indicated by vertical dashed lines) and the maximum (peak) of cytosolic intensity. Amp was defined as the corresponding intensity difference. $T_{r}$ was defined as the time starting from the maximum (peak) to the rest of the response. (A) An exemplary response of cells without self-oscillation before stimulation. (B) An exemplary response of cells showing self-oscillations before stimulation. (C)(E) Histograms of analysis of Amp, $T_{p}$ and $T_{d}$ from cells that did not show oscillations $50 \mathrm{~s}$ before stimulation. (F)-(H) Histograms of analysis of Amp, $\mathrm{T}_{\mathrm{p}}$ and $\mathrm{T}_{\mathrm{d}}$ from cells showing oscillations $50 \mathrm{~s}$ before stimulation. 


\subsection{Responses of self-oscillating cells to external stimuli}

The sensing of cAMP initiates a large amount of actin filaments formation everywhere in the cortical region of $D$. discoideum cells, resulting in a depletion in the cytosolic signal. The following excessive disassembly of actin filaments brings the system back to the basal state (Figure 4.1A). To investigate the influence of the existence of self-oscillation on cAMP responses, we analyzed the responses to stimulation from all cells and examined if the response dynamics depends on whether or not the cells were self-oscillating before stimulation. Considering the fact that the response after stimulation is composed of polymerization, depolymerization and final recovery, the response dynamics was dissected into three successive intervals (Figure 4.1A): $\mathrm{T}_{\mathrm{p}}$ (where polymerization dominates), $\mathrm{T}_{\mathrm{d}}$ (where depolymerization dominates) and $T_{r}$ (the remaining relaxation time). Since the signal becomes unrecognizable after the depolymerization dominated region, we will focus on $T_{p}$ and $T_{d}$. The amplitude (Amp), defined as the difference between the maximum and minimum of the cytosolic signal, will also be investigated to know the amount of depolymerized actin. Figure 4.1C-4.1E shows the analysis for the cells that did not self-oscillate $50 \mathrm{~s}$ before stimulation was applied and $\mathrm{F}-\mathrm{H}$ shows the analysis for the cells that oscillated regularly $50 \mathrm{~s}$ before stimulation. Self-oscillations seems to have no impact on the actin dynamics of cAMP responses.

To gain further insight into the influence of stimulation on the occurrence of selfoscillations, we applied periodic stimuli to self-oscillating cells. Figure 4.2 shows that the external stimulation always immediately replaced the rhythm of the selfoscillating cell. This property was independent of myosin II. Self-oscillations of cells genetically lacking myosin II were also replaced by cAMP responses immediately after the stimulation of cAMP. Cells can re-establish self-oscillation once the impact of stimulation is gone (Figure 4.2C). However, external stimuli can neither eliminate nor trigger the occurrence of self-oscillation: after 10 pulses of periodic stimuli, self-oscillations of cells can either be restored (Figure 4.2D) or stopped (Figure 4.2B) whereas non-self-oscillating cells can either remain non-oscillating (Figure 4.2A) or begin to oscillate (Figure 4.2C). 

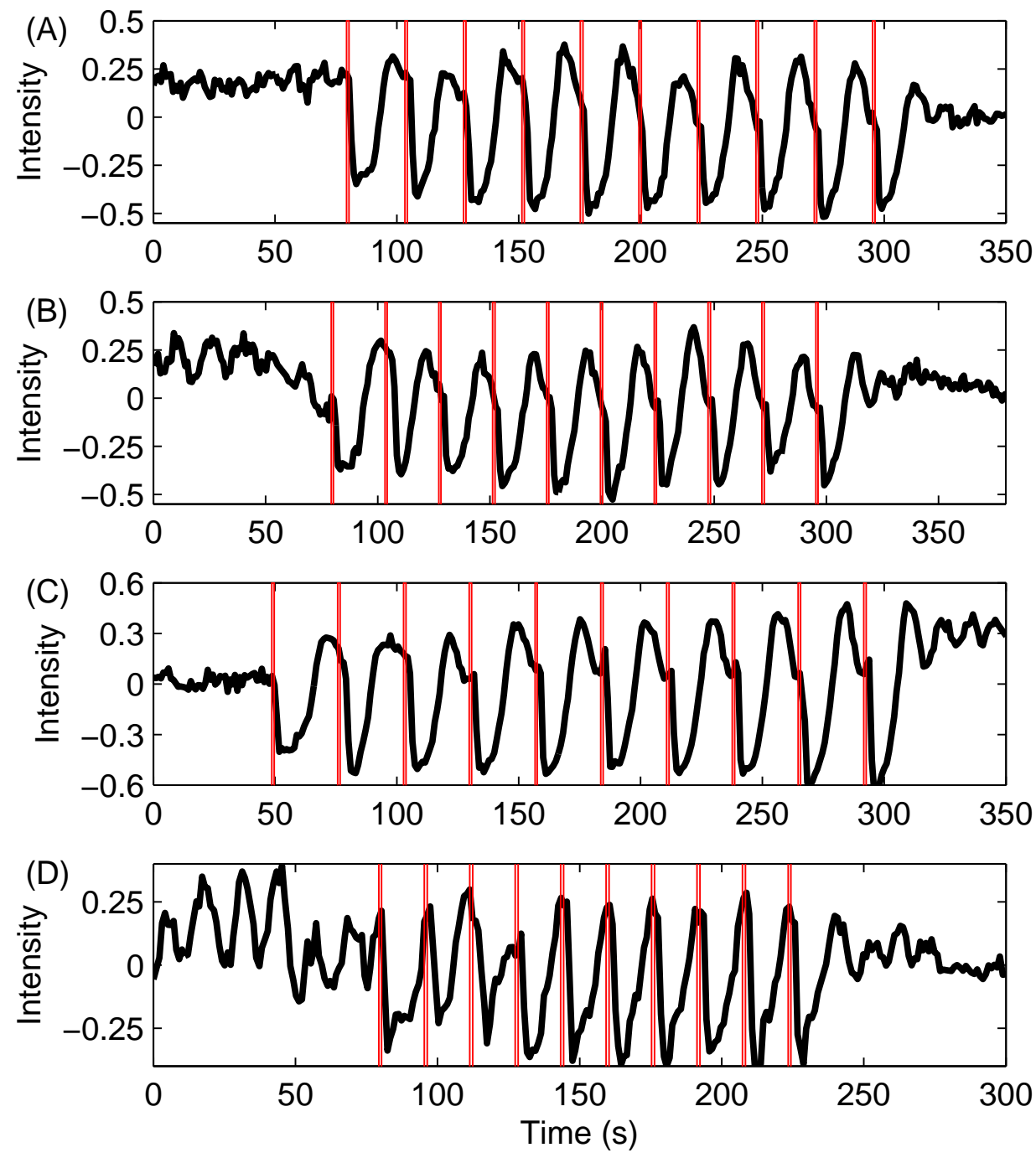

Figure 4.2: The impact of periodic stimulation on self-oscillation. Wild-type cells labeled LimE-GFP were observed. Black traces show the cytosolic signal of LimE-GFP. Red lines show the time when cAMP was applied to the cell. (A) Periodic stimuli were applied with an interval of $24 \mathrm{~s}$. The cell neither shows self-oscillation before nor after the periodic stimulation. (B) Periodic stimuli were applied with an interval of $24 \mathrm{~s}$. The cytosolic signal self-oscillates before the periodic stimulation but ceases after the periodic pulses. (C) Periodic stimuli were applied with an interval of $27 \mathrm{~s}$. The cell did not show self-oscillation before the stimulation but oscillated after the periodic excitation. (D) Periodic stimuli were applied with an interval of $16 \mathrm{~s}$. The cell shows self-oscillations both before and after the periodic stimulation. 


\subsection{Effects of different strengths of stimulation}

As the cytoskeletal network of $D$. discoideum is known to be a nonlinear dynamical system [110] and one of the characteristics of nonlinear systems is the dependence of the response frequency on the excitation strength [139], we decided to stimulate the cells with different doses of cAMP to understand the effect of perturbation. The stimulation strengths were controlled by adjusting the concentration of BCMCMcaged cAMP flowed in and with the power of uncaging laser fixed. Figure 4.3 shows that the response amplitude only increases with the stimulation strengths in a very narrow region. This implies that there is a threshold, or a narrow concentration range of cAMP, at which the cell becomes responsive. It has been observed that the upstream $\mathrm{PH}-\mathrm{Crac}$ signals increased monotonically with increasing cAMP concentration only within a very limited range (around $10^{-9}-10^{-8} \mathrm{M}$ ), below which the cells were essentially nonresponsive. Above $10^{-8} \mathrm{M}$, the responses were rather scattered, although the average values seemed to indicate a plateau [140]. Considering the fact that the uncaging efficiency of BCMCM-caged cAMP is around 1\% [115, 116], the response threshold seems to be well preserved from signaling pathways to actin networks.

We next want to know if stimulation strength has any effect on the response frequency. However, it is difficult to precisely determine the time at which the first oscillation after the stimulation ends. The best way to capture the oscillation frequency is to stimulate the cells periodically to find the resonance frequency. As the response amplitude reaches maximum when the intrinsic frequency matches the frequency of external stimulation, the intrinsic frequency (resonance frequency) can be extracted by recording the amplitude of cellular responses to stimulation with different periods. With this method, Westendorf et al., found that the resonance period of actin dynamics is at $20 \mathrm{~s}$ [109]. To confirm the resonance shift due to external stimulation, we stimulated the cells periodically with stronger stimulation strength. As the caged cAMP used here (BCMCM-caged cAMP) releases active cAMP more efficiently than that used in [109] (DMNB-caged cAMP) [115, 116], BCMCMcaged cAMP released more functional cAMP than DMNB-caged cAMP at identical concentrations $(10 \mu \mathrm{M})$. Figure 4.4 shows that the resonance peak from stronger stimulation has a bigger amplitude as well as a longer period. 

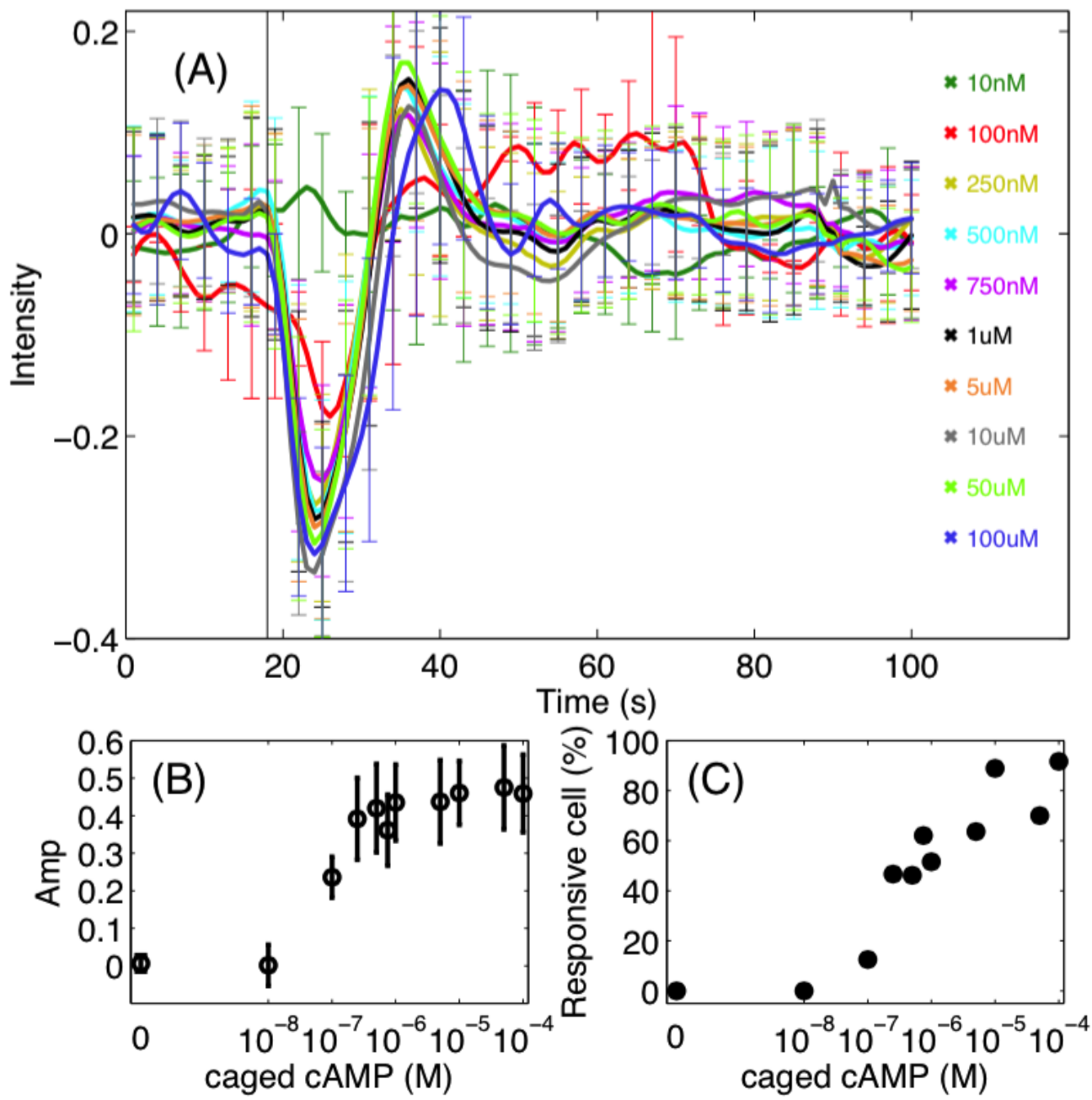

Figure 4.3: Response to external stimulation depends on the strength of excitation. The LimEGFP signal from wild-type cells were observed. Different stimulation strengths were applied by fixing the power of uncaging laser to $100 \%$ but the concentration of BCMCM-caged cAMP continuously flowing through the channel was changed. (A) Average responses from more than 10 cells stimulated at the indicated concentration of BCMCM-caged cAMP. Error bars show standard deviation. Vertical black line shows the time point cAMP was applied. (B) Quantification of intensity change in (A). Amp is defined in Figure 4.1 and error bars show standard deviation. (C) Percentage of responsive cells at different stimulation strength. 


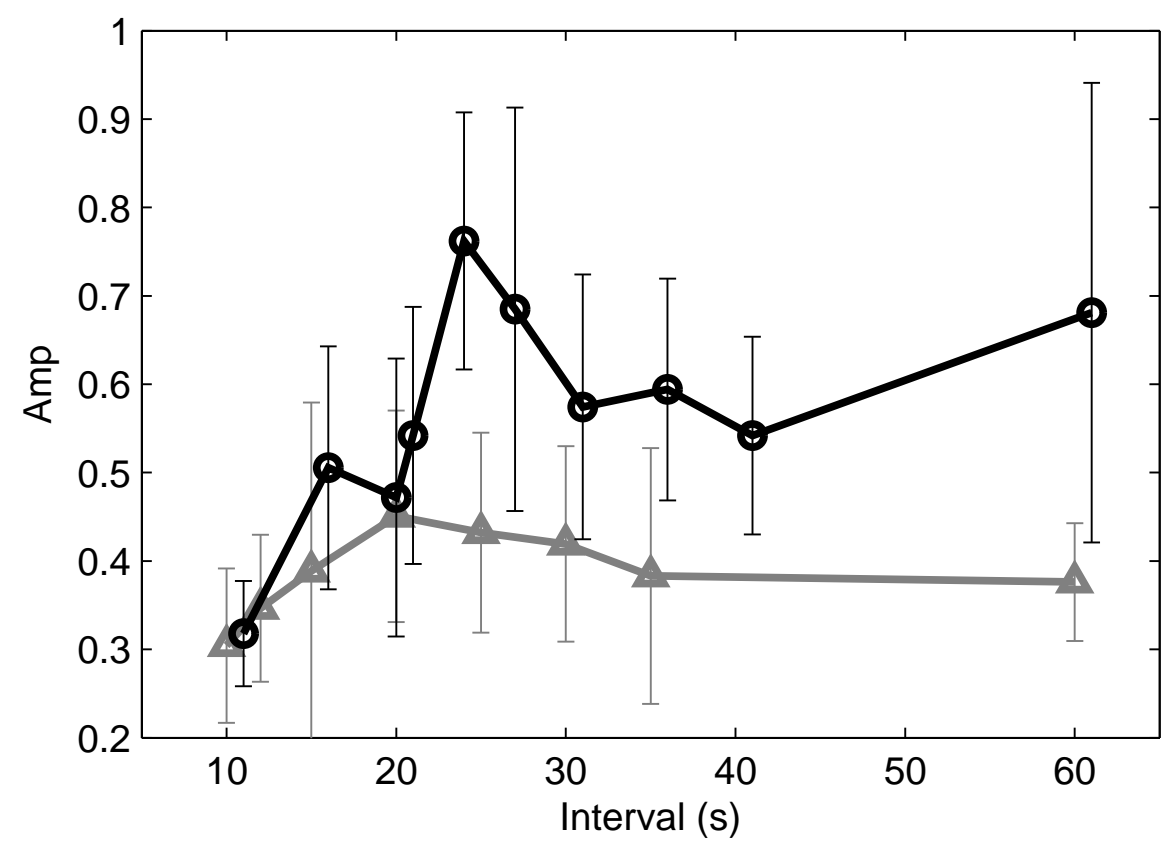

Figure 4.4: Resonance peak shifts for different stimulation strength. At the specified interval we applied 10 stimuli to each cell and analyzed the amplitude of individual responsive traces. At least 20 cells were observed for every interval. Black line shows the resonance curve for strong stimulation (10 $\mu \mathrm{M} \mathrm{BCMCM})$. Circles refer to stimulation intervals done in the experiment and the error bars show the standard deviation of the response amplitude in different cells. Gray line shows the resonance curve at weak stimulation $(10 \mu \mathrm{M} \text { DMNB })^{1}$

Transition of the actin dynamics in self-oscillating cells to a cAMP response induced by external stimulation of cAMP shows that self-oscillation is immediately replaced by stimulated response, which requires longer time to finish one oscillation compared to self-oscillations (Figure 4.1B). Therefore, when we stimulated the cells periodically, the resonance period peaks at a time longer than the period of self-oscillations.

\footnotetext{
${ }^{1}$ As the data from [109] were analyzed differently (e.g. traces of cells were not normalized according to the intensity before stimulation to exclude the effect from different expression levels among cells; all responsive traces were averaged first and then analyzed so the information about variation among cells cannot be seen from the resonance curve.) We took the data and reanalyzed it based on the scope of analysis used here.
} 


\subsection{Single cell experiments}

The error bars on the resonance points are due to the variations among cells. It is known that even under the same strength of stimulation, the responses from different cells vary a lot [141]. To gain more insight into the cell-cell diversity, we designed a new experiment where we successively apply cAMP at different concentrations to the same cell by adjusting the power of the uncaging laser with an interval of $4 \mathrm{~min}$ between sequential stimuli to allow the cells to fully recover from the previous cAMP exposure. The stimulation was always started with $100 \%$ laser power and gradually decreased to the point where the cells no longer responded; the laser intensity was then increased again to confirm that the unresponsive signal was due to the weak stimulation rather than cellular damages. This design enables us to explore questions like "Do all cells have the same threshold?" and "Does the response stay constant after reaching the plateau?"

Laser Power (\%)

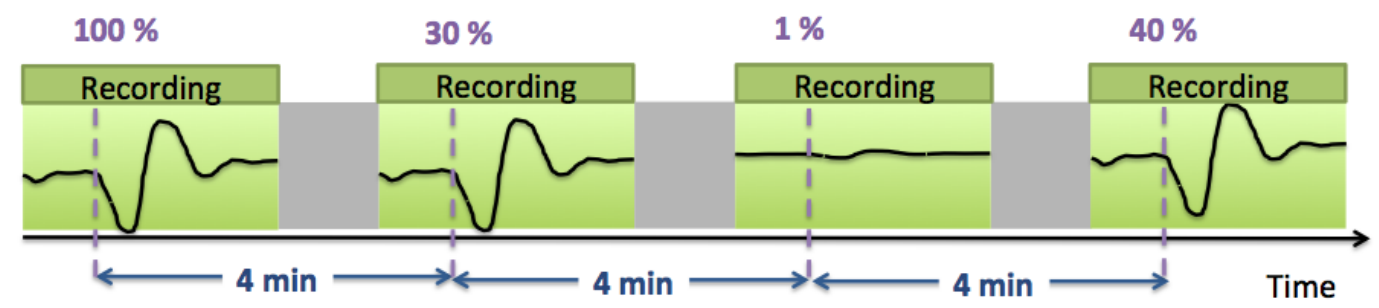

Figure 4.5: A schematic diagram illustrating the experiments that apply different doses of cAMP to single cells. The concentration of BCMCM-caged cAMP flowed in the channel was fixed to $100 \mathrm{nM}$. By altering the power of the uncaging laser, different doses of cAMP can be applied to the same cell. The stimulation always started from $100 \%$ power of uncaging laser and then gradually decreased till the point the cell did not respond anymore. We then increased the power of uncaging laser again to make sure that the reason the cell did not respond was due to weak stimulation rather than cell damages. The laser power shown here is just to illustrate the idea of decrease and then increase again. The actual values of laser power were adjusted appropriately for each individual cell. Every stimulus was separated $4 \mathrm{~min}$ to make sure the cell was fully recovered from previous stimulation. The green regions mark the time cells were under recording whereas the gray regions mark the time without any recording. 
Figure 4.6 show a distinct cell-to-cell variability in the response threshold; some cells respond to very weak stimulation ( $0.3 \%$ laser power, Figure $4.6 \mathrm{~A})$, whereas the upper threshold for responsiveness can sometimes reach $80 \%$ laser power (Figure 4.6B).
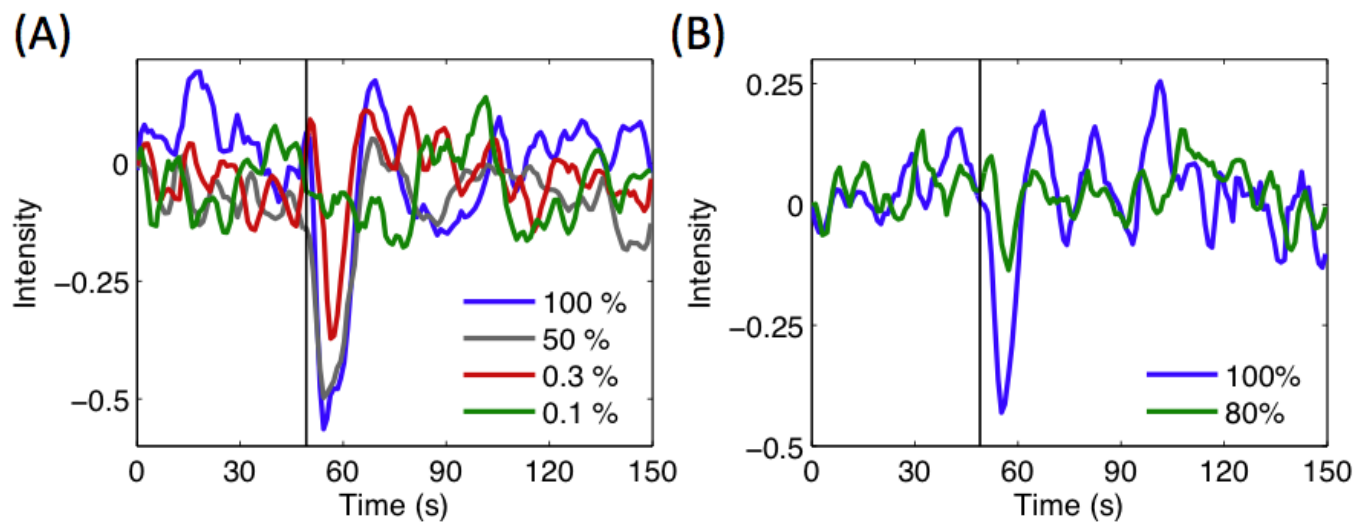

Figure 4.6: Threshold for cAMP responses varies a lot among cells. (A) Exemplary responses to different stimulation strength in one cell. Overlay of Different colors show the responses to different power of laser. Black line indicates the time uncaging laser was applied for $1 \mathrm{~s}$. (B) Another exemplary response to different stimulation strength in one cell.

The stimulation strength dependent response amplitude varies from cell to cell. Some cells increased their response amplitude as the stimulation strength grows but in some cells there is no apparent correlation between the stimulation strength and Amp (Figure 4.7A). Considering the fact that we can observe cells increase their response amplitude with stimulation strengths only in a very narrow region of cAMP concentration and the threshold may differ from cell to cell, the cells without apparent correlations may have low thresholds, due to which the concentrations of caged cAMP applied here were always above their thresholds. If this is the case, then the scattered responses should be observed for all cells when they are stimulated with very strong stimulation, which reaches the plateau of all cells. We therefore flowed 100 times higher concentration of caged-BCMCM cAMP into the microfluidic channel to apply the strong stimulation. Figure 4.7B shows that all cells show scattered responses at different stimulation strengths. The scattered behavior might come from the varying stimulation strengths in the plateau region or it could be an intrinsic property of the cells. To distinguish these between two possibilities, we stimulated 
one cell 5 times using an identical amount of cAMP. Figure 4.7C shows that the responses scattered in a similar range once cells crossed the response threshold, confirming that the scattering of amplitude response originates from the intrinsic noise of the cells.
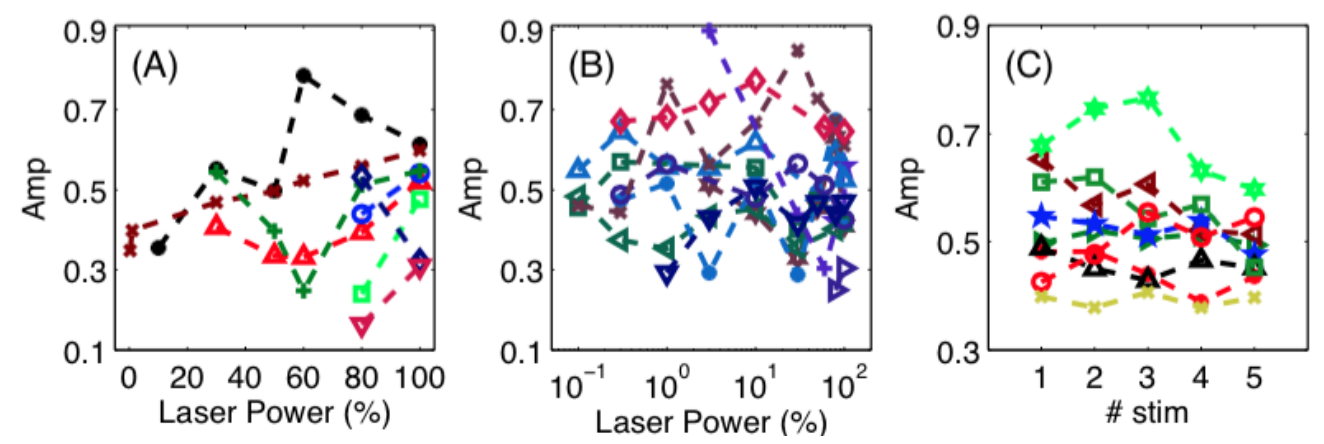

Figure 4.7: Intrinsic noise causes the scattering of response amplitude. Wild-type cells labeled with LimE-GFP were observed with the method shown in Figure 4.5. Different symbols show the response from different cells. (A) The variation of amplitude response at different strengths of external stimulation. Experiments were done with $100 \mathrm{nM}$ BCMCM-caged cAMP continuously flowing in the channel. Laser intensity was adjusted according to the x-axis. (B) The variation of amplitude response at different strength of external stimulation. Experiments were done with $10 \mu \mathrm{M}$ BCMCM-caged cAMP continuously flowing in the channel. Laser intensity was adjusted according to the $\mathrm{x}$-axis. (C) The variation of amplitude response when cells were stimulated with the same strength of external stimulation is shown. Experiments were done with $10 \mu \mathrm{M}$ BCMCM-caged cAMP continuously flowing in the channel and the laser intensity was always 100\%. Interval between successive stimulation was $100 \mathrm{~s}$.

To explore the mechanism that governs the response frequency at different stimulation strengths, we analyzed how the polymerization and depolymerization times change with stimulation strength in different cells. Figures $4.8 \mathrm{~A} \& 4.8 \mathrm{~B}$ shows that under all ranges of stimulation, polymerization time remains scattered around a constant value without a clear trend of variation with stimulation strength. The polymerization time was also scattered in cells responding to the same stimulation (Figure 4.8C), suggesting it is an intrinsic propertyof the cells. On the other hand, depolymerization time shows a trend similar to the response amplitude (Figure 4.8D to 4.8E); depolymerization time grows with the stimulation strength in some cells, whereas in some cells no clear trend was observed. 

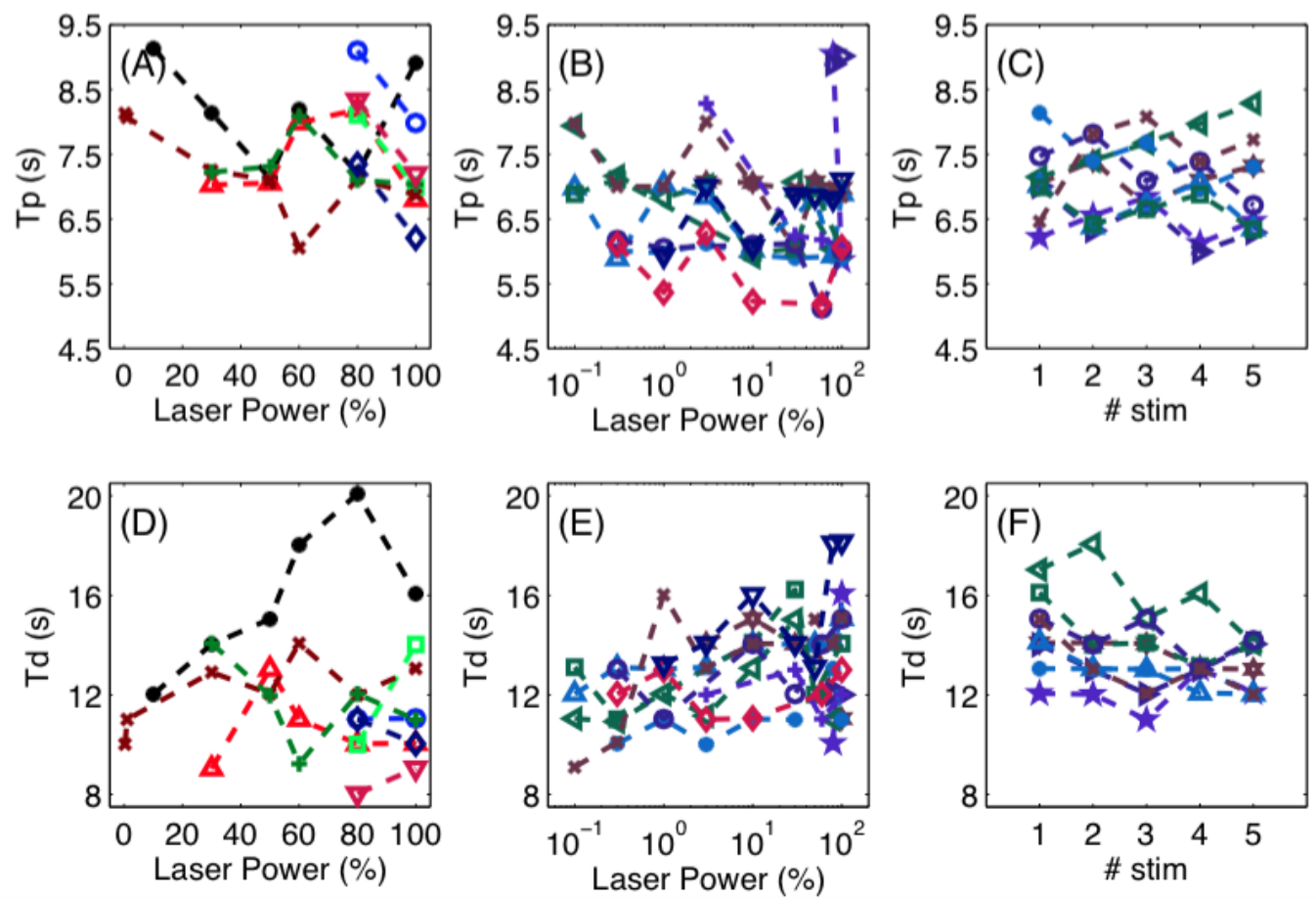

Figure 4.8: $T_{p}$ and $T_{d}$ at diverse stimulation strength in different cells. Wild-type cells labeled with LimE-GFP were observed with the method shown in Figure 4.5. Different symbols with different colors show the response from different cells. (A), (D) The variation of $T_{p}$ and $T_{d}$, respectively, at different strengths of external stimulation. Experiments were done with $100 \mathrm{nM}$ BCMCM-caged cAMP continuously flowing in the channel. Laser intensity was adjusted according to the $\mathrm{x}$-axis. (B), (E) The variation of $\mathrm{T}_{\mathrm{p}}$ and $\mathrm{T}_{\mathrm{d}}$, respectively, at different strength of external stimulation. Experiments were done with $10 \mu \mathrm{M} \mathrm{BCMCM-}$ caged cAMP continuously flowing in the channel. Laser intensity was adjusted according to the $\mathrm{x}$-axis. (C), (F) The variation of $\mathrm{T}_{\mathrm{p}}$ and $\mathrm{T}_{\mathrm{d}}$, respectively, when cells were stimulated with the same strength of external stimulation are shown. Experiments were done with $10 \mu \mathrm{M}$ BCMCM-caged cAMP continuously flowing in the channel and the laser intensity was always $100 \%$. Interval between successive stimulation was $100 \mathrm{~s}$. 


\subsection{Proposed underlying mechanism}

In spite of the variations inside every cell and the prominent variability among cells, responses from all cells reveal the same trend when we plotted the polymerization time and the depolymerization time with the corresponding response amplitude: responses with scattered amplitudes have their polymerization times scattered around a constant value (Figure 4.9A), whereas responses with larger amplitudes also show longer depolymerization times (Figure 4.9B).

It has been observed that the amount of capping protein peaked at the same time as F-actin after the stimulation by cAMP and their amounts then decreased together [127], suggesting that it is the binding of capping protein that terminates the polymerization process. We therefore regarded $T_{p}$ as the binding time of capping protein.
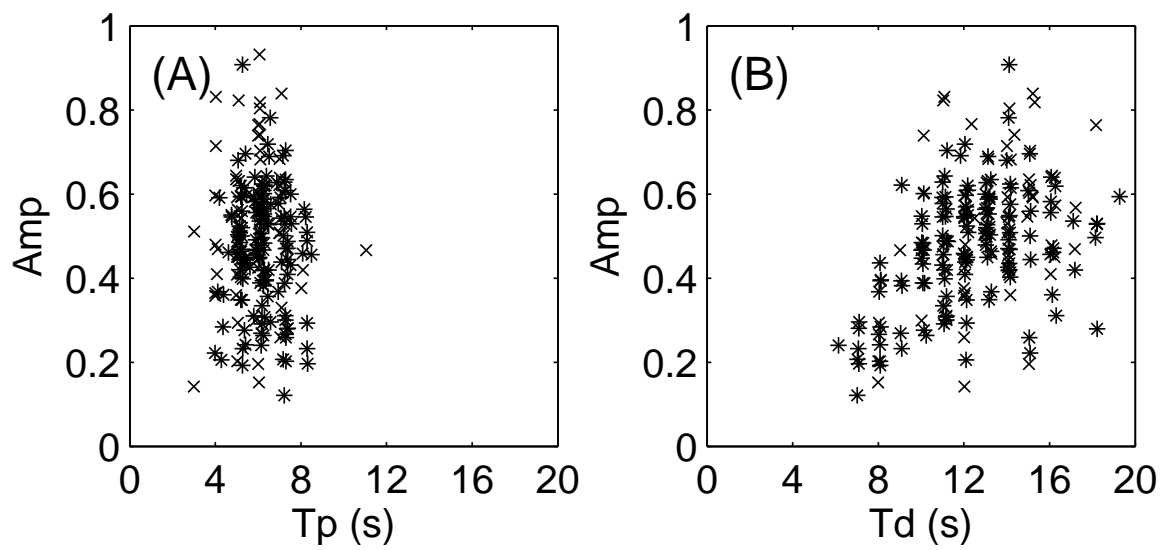

Figure 4.9: Global parameters that determine the behavior of cells. Wild-type cells labeled with LimE-GFP were observed. Black dots show the analysis from 314 selfoscillating cells and gray crosses show the analysis from 95 cells responding to a single $1 \mathrm{~s}$ stimulus at $100 \%$ laser power to uncage $10 \mu \mathrm{M}$ BCMCM-caged cAMP. Black stars show the analysis from 136 cells responding to a single $1 \mathrm{~s}$ stimulus of different concentrations. The stimulus was applied with $100 \%$ laser power to uncage different concentration of BCMCM-caged cAMP (from $30 \mathrm{nM}$ to $100 \mu \mathrm{M}$ ). (A) Relation between Amp and $\mathrm{T}_{\mathrm{p}}$. (B) Relation between Amp and $\mathrm{T}_{\mathrm{d}}$. 
To explore how the fixed polymerization time leads to the diverse response amplitude (Figure 4.9A), we examined how the response amplitude changes with the parameters controlling polymerization and depolymerization: first, it is known that proteins accelerating depolymerization like Aip1 and Coronin showed cAMP response similar to the actin filaments but with a delay $(\tau)$ distributed around $3 \mathrm{~s}$ [109, 110, 117]. Although a later activation of depolymerization proteins (i.e., longer $\tau$ ) might allow faster polymerization, the delay time $(\tau)$ between LimE and Aip1 or Coronin was found to be uncorrelated with Amp (Figure 4.10A). Secondly, it is known that extracellular stimuli can activate Arp2/3 via activating WASp/Scar [128131]. As the association rate of monomeric actin to actin filaments is constant, more activated Arp2/3 allows more filamentous actin to grow simultaneously, resulting in a faster apparent polymerization rate. Observation of cells co-expressed with Arp3-GFP and LimE-mRFP shows a correlation between the response amplitude of Arp2/3 and LimE, suggesting that the distribution of Amp responses originated from different amount of activated Arp2/3 (Figure 4.10B).
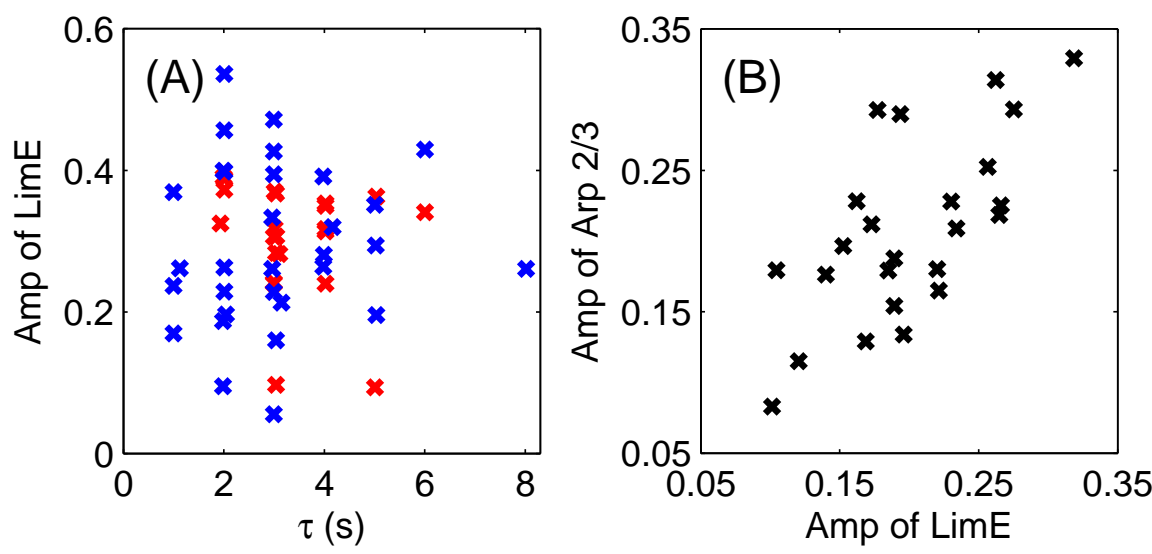

Figure 4.10: The dependence of response amplitude (Amp) on polymerization and depolymerization. Since the responses from Coronin, Aip1 and Arp2/3 are all similar to LimE dynamics after stimulation, Amp from different labels of different cells were analyzed according to the definition of Amp in Figure 4.1. (A) Amp is independent of the delay time $(\tau)$ between LimE and depolymerization proteins. Each cross shows the response from one cell to a single $1 \mathrm{~s}$ stimulus of cAMP. The stimulus was applied with $100 \%$ laser power to uncage $10 \mu \mathrm{M}$ BCMCMcaged cAMP. Blue crosses show the analysis of 25 cells co-expressed LimE-mRFP and Aip1-GFP. Red crosses show the analysis of 32 cells co-expressed LimEmRFP and Coronin-GFP. (B) Amp was correlated with the response amplitude of Arp2/3. 23 cells co-expressed LimE-mRFP and Arp3-GFP were observed. Each cross shows the response from a single cell to a single $1 \mathrm{~s}$ stimulus. The stimulus was applied with $100 \%$ laser power to uncage $10 \mu \mathrm{M}$ BCMCM-caged cAMP. 
The correlation between Amp and $T_{d}$ was surprisingly robust. Amp of the cAMP responses of different cells to the same stimulation strengths (crosses in Figure 4.9), to different stimulation strength (stars in Figure 4.9) as well as self-oscillating cells (dots in Figure 4.9) all lay on the same curve, indicating that all the responses were governed by the same underlying mechanism: it takes longer to depolymerize more actin filaments. The reason behind this machinery is simply due to the fact that F-actin can disassemble only from the pointed end of a filament.

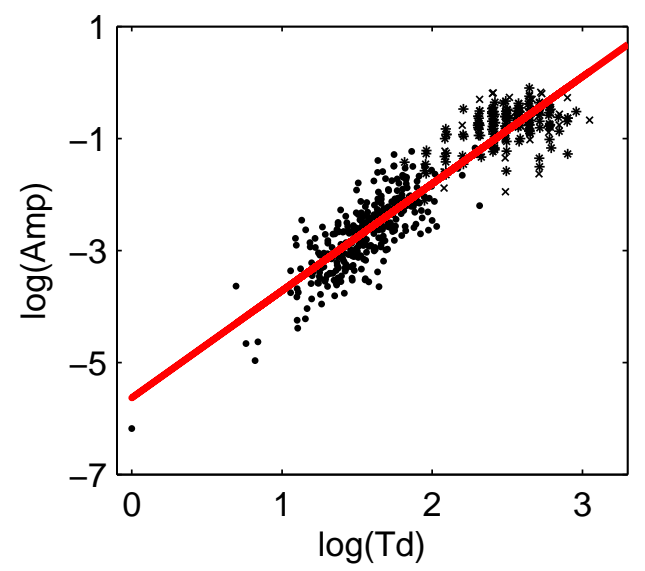

Figure 4.11: The relation between Amp and $T_{d}$ on a log-log scale. Wild-type cells labeled with LimE-GFP were observed. Black dots show the analysis from 314 selfoscillating cells and gray crosses show the analysis from 95 cells responding to a single $1 \mathrm{~s}$ stimulus at $100 \%$ laser power to uncage $10 \mu \mathrm{M}$ BCMCM-caged cAMP. Black stars show the analysis from 136 cells responding to a single $1 \mathrm{~s}$ stimulus of different concentrations. The stimulus was applied with $100 \%$ laser power to uncage different concentrations of BCMCM-caged cAMP (from $30 \mathrm{nM}$ to $100 \mu \mathrm{M})$. Red line shows: $\log (\mathrm{Amp})=-5.74+1.99 \times \log \left(\mathrm{T}_{\mathrm{d}}\right)$.

With the assistance of severing proteins such as ADF/cofilin, more actin filaments can be disassembled at the same time (i.e., Amp grows faster than the increase of $\mathrm{T}_{\mathrm{d}}$ ). The logarithmic fitting shows that $\mathrm{T}_{\mathrm{d}}$ grows as $A m p^{0.50}$ (Figure 4.11), reflecting the role of severing in the depolymerization process. Therefore. an Amp- $T_{d}$ curve reflects the state of actin depolymerization. To explore how the curve changes if some components of the depolymerization machinery are disabled, we next observed the response dynamics in cells genetically modified lacking Aip1. Although some points in the data from Aip1-null cells are indistinguishable from wild-type cells, the entire data set of Aip1-null cells is shifted to the right compared to wild-type cells, i.e., $\mathrm{T}_{\mathrm{d}}$ grows slower with Amp compared to wild-type cells (Figure 4.12A). As there are many other components such as Coronin and $\mathrm{ADF} /$ cofilin responsible for 
depolymerization $[27,29,34]$, it is possible that they can compensate for the lost function of Aip1. Since lacking ADF/cofilin is lethal to cells [36, 142], experiments with cells without Coronin or both Aip1 and Coronin might help to support our hypothesis.

It is worthy to note that Aip1-null cells deviated more from wild-type cells at higher Amp. This implies that Aip1 is indispensible for effective actin turnover for a huge actin network. We also found that the overlap between the data of Aip1-null and wild-type seems to increase with the starvation time of Aip1-null cells (Figure 4.12B).
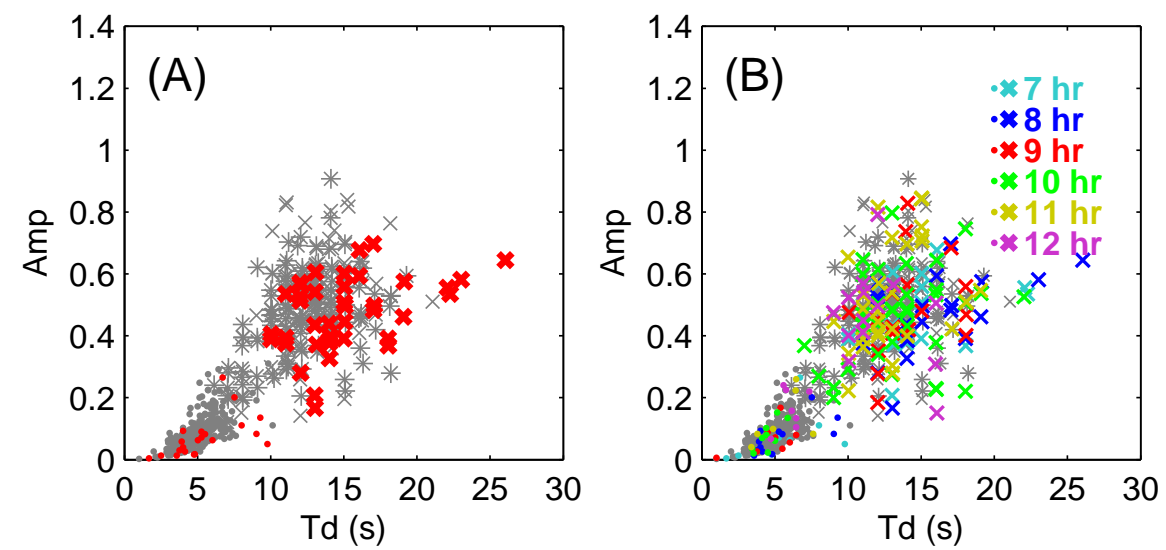

Figure 4.12: Amp- $\mathrm{T}_{\mathrm{d}}$ relation from Aip1-null cells labeled with LimE-GFP. Dots show analysis from self-oscillating cells. Crosses and stars show the analysis from cells responding to a single $1 \mathrm{~s}$ stimulus. (A) Comparison between wild-type $(\mathrm{N}=545)$ and Aip1-null cells $(\mathrm{N}=188)$. Data plotted in gray are the same as the data in Figure 4.11A. Red dots show data from 21 Aip1-null cells and red crosses show data from 41 Aip1-null cells starved within 8 hours. (B) Effect of starvation in Aip1-null cells. Different colors show the data from different starvation times. 


\subsection{Nonlinear properties of responses}

As the cAMP responses resemble the oscillation of a damped oscillator, we next tried to describe the response with the dynamics of a damped oscillator:

$$
\ddot{x}+2 \xi \dot{x}+k x=F(t),
$$

We regarded the amplitude $\mathrm{x}$ of damped oscillators as the response amplitude of the cytosolic signal after the stimulation of cAMP; the application of cAMP to the system was considered as the application of a force $\mathrm{F}$ with a Heaviside step function that stays at a constant value for $1 \mathrm{~s}$; $\xi$ is the damping coefficient.

Simulations of periodic stimuli shows that the position of the resonance peak did not change under the stimulation of different strength (Figure 4.14).

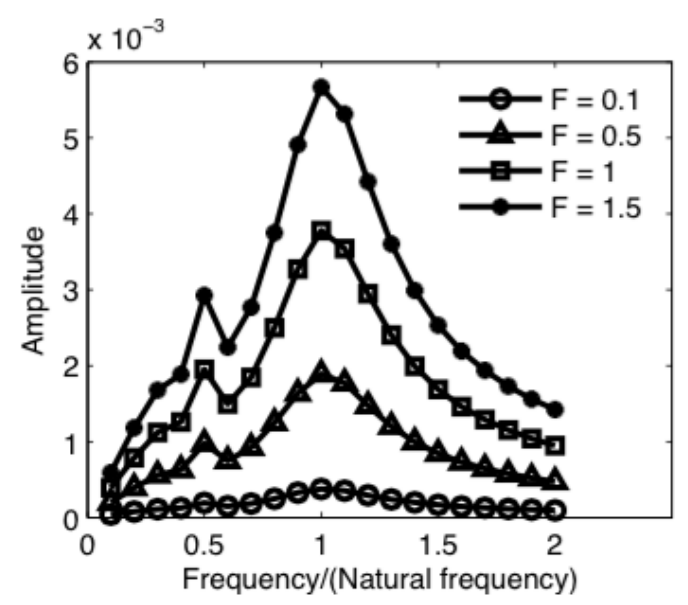

Figure 4.13: Simulated resonance curves of damped oscillators. Simulations were conducted with equation 4.1. Frequency of stimuli shown in the $\mathrm{x}$-axis was normalized to the resonance frequency when $\gamma=0$ (Natural frequency). Different symbols display different amplitude of applied force (F).

To reproduce the nonlinear phenomena observed in the experiments that response frequency is dependent on the stimulation strength, a cubic term was added to the damped oscillator. It is also known as duffing oscillators [139]. $\gamma$ is the parameter introducing nonlinearity:

$$
\ddot{x}+2 \xi \dot{x}+k x+\gamma x^{3}=F(t),
$$


Simulations with periodic stimuli support the nonlinear nature of the phenomenon observed in experiments (Figure 4.4). Different strengths of the external stimuli always changed the responsive amlitude but the resonance frequency only varied when $\gamma$ was nonzero (Figure 4.14A). $\gamma$ shifts the resonance peak by introducing a nonlinearity to the damped oscillators: the magnitude of $\gamma$ (along with F) reflects the extent of resonance peak shift and the sign of $\gamma$ reflects the direction of the shift (Figure 4.14C).
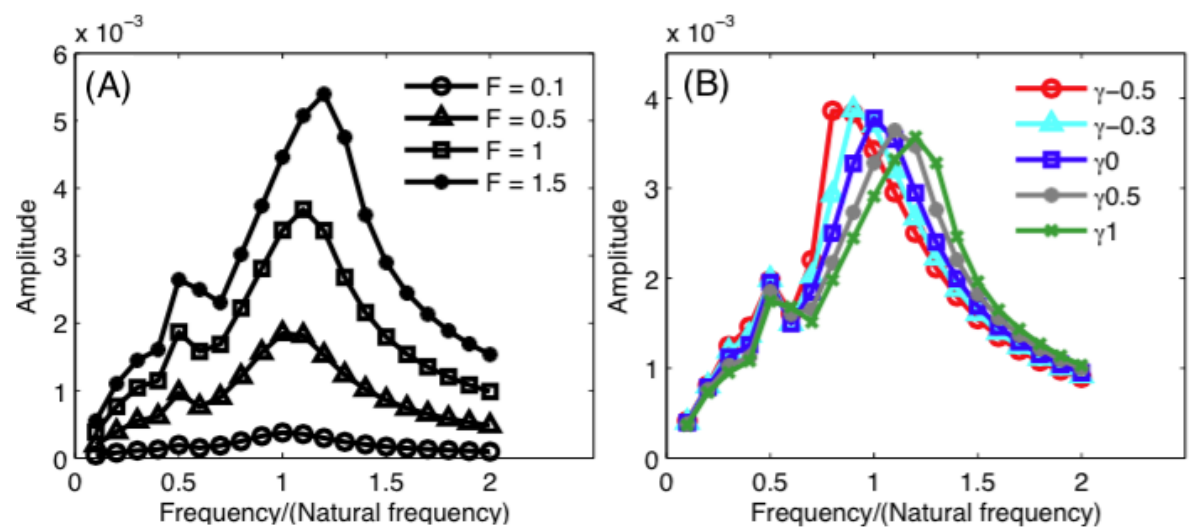

Figure 4.14: Effect of altering the strength of stimulation on the resonance curve. Simulations were conducted with equation 4.2. Frequency of stimuli shown in the $\mathrm{x}$-axis was normalized to the resonance frequency when $\gamma=0$ (Natural frequency). (A) Simulated resonance curves of duffing oscillators $(\gamma=0.4)$ at different forces of stimulation. Different symbols display different amplitude of applied force. (B) Effect of altering the values of $\gamma$ on the resonance curve. Different colors show resonance curves with different $\gamma$ values.

However, the parameters of duffing oscillators have no identifiable physiological counterparts in terms of actin dynamics as they were not made to study actin dynamics in the first place. A time delayed feedback model has been proposed to explain the actin dynamics based on a time-delay between the polymerization and depolymerization [109]:

$$
\begin{gathered}
\frac{\mathrm{dA}(\mathrm{t})}{\mathrm{dt}}=k_{+}(t) A(t)-k_{-} A(t) A(t-\tau), \\
k_{+}(t)=k_{+}^{0}+k_{s}(t),
\end{gathered}
$$

where $k_{+}(t)$ is the overall polymerization rate which is regulated by intrinsic polymerization $\left(k_{+}^{0}\right)$, external stimulation $\left(k_{s}(t)\right)$ and adaptation; $A(t)$ is the concentration of actin filaments (i.e., the cortical fluorescence intensity observed in our experiment); $k_{-}$is the overall depolymerization rate of the system; $\tau$ is the delay time between 
polymerization and depolymerization. The solutions (fixed points) are stable when $k_{+}^{0} \tau<\pi / 2$, where the oscillations decrease amplitude with time; solutions become unstable as $k_{+}^{0} \tau>\pi / 2$, where the oscillations increase amplitude with time and finally enter time-periodic limit cycle oscillations. The system thus encounters a Hopf bifurcation at $k_{+}^{0} \tau=\pi / 2$, where the solutions oscillate with constant amplitude.

This model reproduces several characteristic features of the in vivo situation: first, it has been shown that four times the delay time is the oscillation period when the parameters are at the bifurcation point [143]. Secondly, we were able to reproduce the stimulation strength dependence of the resonance curve in the frame work of this model (Figure 4.15).

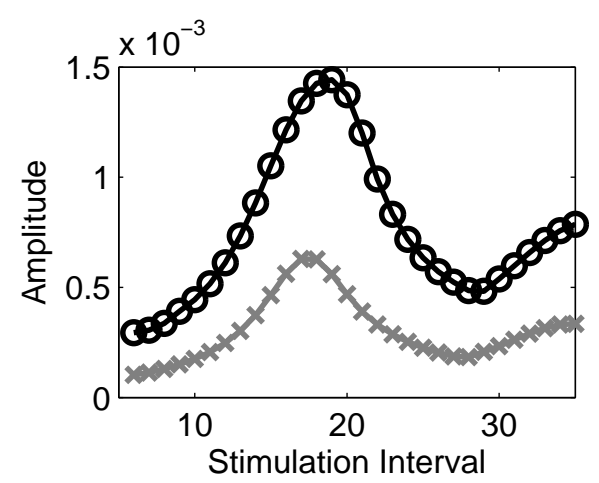

Figure 4.15: Simulations of resonance curves with different stimulation strengths. Resonance curves simulated by time delayed feedback model (Eq. 4.2) with $k_{+}^{0}=0.3$ and $\tau=4$. Stimulation $\left(k_{s}(t)\right)$ was applied as Gaussian function with maximum value of 1.5 (black curve) and 0.6 (gray curve).

Here we further examined the model by comparing simulations with our experimental results. First, oscillating solutions last longer as the parameters come close to the bifurcation point, implying the intrinsic parameters of self-oscillating cells are close to the bifurcation point, where the oscillation period is close to four times the delay time $(\tau)$. The distribution we report here in Figure 3.5 agrees well with four

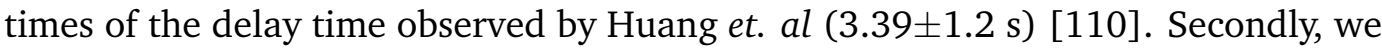
observed that the resonance frequencies depend on the strength of external stimulation; simulation at different strength of the stimulation reveals a shift to longer period in the resonance curve (Figure 4.4).

However, we would like to stress that Eq. 4.3 is based on the assumption that the amount of depolymerization proteins always increase with the polymerized filamentous actin but without a limit. According to Eq. 4.3, increasing the amount of depolymerization proteins not only increases the depolymerization rate but also 
depletes the actin pool, resulting in an extremely slow growth rate and prolongs the recovery time tremendously. As a result, this model failed in predicting how the deploymerization time $\left(\mathrm{T}_{\mathrm{d}}\right)$ changes with response amplitude (gray lines with triangles in Figure 4.16C).
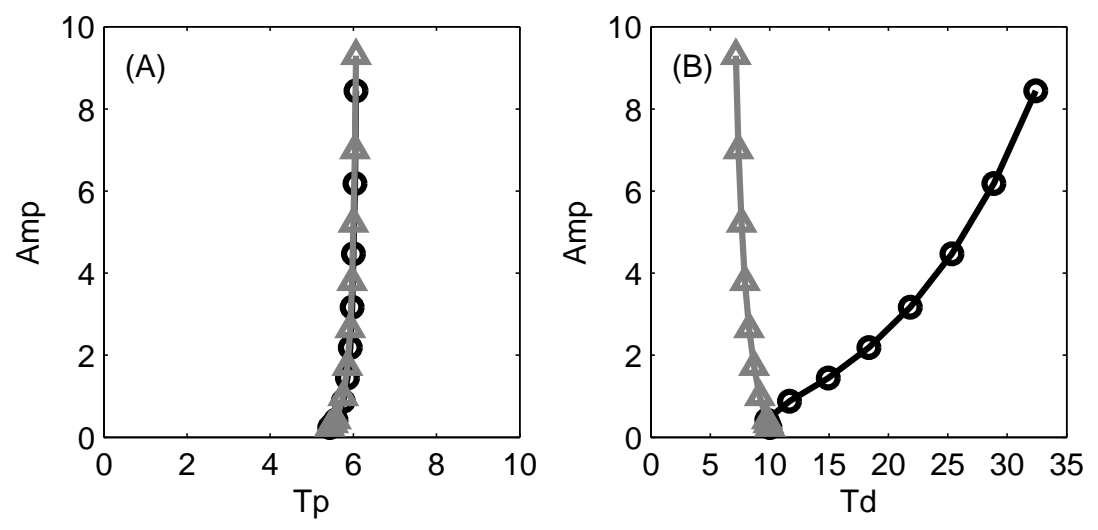

Figure 4.16: Simulation of Amp versus $T_{p}$ and Amp versus $T_{d}$ at different strengths of external stimulation. Gray lines show simulations from time-delayed model proposed in reference [109] (Eq. 4.2) with $k_{+}^{0}=0.3$ and $\tau=4$. Black lines show simulations from the model with depolymerization threshold (Eq. 4.6) using $k_{+}^{0}=0.3$ and $\tau=4$. Stimulation $\left(k_{s}(t)\right)$ was applied as Gaussian function with a maximum value of $0.3,0.4,0.5,1.0,1.5,2.0,2.5,3.0,3.5$, and 4.0. (A) Amp versus $T_{p}$. (B) Amp versus $T_{d}$

To include the limited increase of the depolymerization proteins, we modify the time delayed model by setting a threshold to the depolymerization part of Eq. 4.3:

$$
\begin{aligned}
& \frac{\mathrm{dA}(\mathrm{t})}{\mathrm{dt}}=k_{+}(t)+A(t)-k_{-} A(t) B(t), \\
& B(t)= \begin{cases}A(t-\tau) & : A(t-\tau)<C_{\text {thre }}, \\
C_{\text {thre }} & : A(t-\tau) \geq C_{\text {thre }} .\end{cases}
\end{aligned}
$$

An upper limit was set for the maximum amount of depolymerization proteins that can participate in disassembling actin filaments $\left(C_{t h r e}\right)$. Below the threshold concentration, the amount of depolymerization proteins was $A(t-\tau)$, namely, proportional to the amount of actin filaments $A(t)$. Under a strong excitation, $A(t)$ increases according to the time delayed model but instead of growing without limit, the maximum amount of depolymerization protein can be used is the threshold. Simulations show that this modification lead to results consistent with our experimental observation: $T_{d}$ grows with increasing Amp, whereas $T_{p}$ remains constant (black lines with circular symbols in Figure 4.16). 


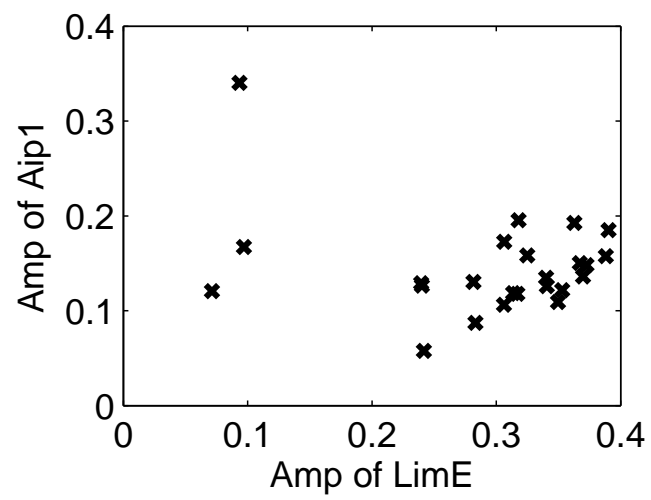

Figure 4.17: Amount of Aip1 is limited with the increasing of LimE. Stimulation was applied at $100 \%$ laser power to uncage $10 \mu \mathrm{M}$ BCMCM-caged cAMP. Response amplitudes from 24 cells co-expressing LimE-mRFP and Aip1-GFP to a single $1 \mathrm{~s}$ stimulation were analyzed. Amp is defined as in Figure 4.1 from the corresponding signal.

It is worthy to note that the diverse amount of activated Arp2/3 can be related to the different polymerization rates $\left(k_{+}^{0}\right)$ in the model, resulting in various response amplitude among cells even under the same stimulation strength. If the modification of the model was correct, then under very strong stimulation, the amount of depolymerization protein will reach a constant value for all cells with various response amplitude. Therefore, we examined the amplitude response of cells co-expressed with LimE-mRFP and Aip1-GFP experimentally. We stimulated cells with a stimulation strength strong enough for all cells to be in the plateau region (i.e., the same stimulation strength as Figure 4.7B). Figure 4.17 shows that cells with varying Amp of LimE all have Aip1 responses in the similar range, supporting the modification of the model. 


\subsection{Conclusion}

By applying cAMP to individual cells, we found that external stimulation leads to a rapid increase in filamentous actin formation in the cortex. As it also required a longer time to depolymerize more actin filaments, the time to finish one oscillation was thus prolonged compared to the actin self-oscillation cycle (Figure 4.1B). This prolongation accounts for the difference between the frequency of the self-oscillations and the forcing resonance peak (Figure 4.4). Comparisons between actin dynamics before and after stimulation suggest that self-oscillations have no effect on cAMP responses and the external stimuli can either trigger or inhibit the occurrence of self-oscillation (Figure 4.2).

To investigate the underlying mechanism, we analyzed how external stimuli change the different parts of the cAMP response. First, the time for actin to polymerize $\left(\mathrm{T}_{\mathrm{p}}\right)$ was the same in self-oscillations as well as responses to external simulation (Figure 3.11 and Figure 3.10), suggesting that this process was controlled by the binding of capping protein. As Arp2/3 can be activated through extracellular stimuli and the consequent actin branch structure enables more actin to bind, simultaneously, during the same polymerization time $\left(\mathrm{T}_{\mathrm{p}}\right)$, cells responding to the stimulation grew actin filaments much faster than self-oscillating cells (compare the distribution of Amp between crosses and dots in Figure 4.9B). Second, the time for filamentous actin to depolymerize prolonged a lot compared to self-oscillating cells (compare the distribution of $T_{d}$ between crosses and dots in Figure 4.9B). It is the prolongation of depolymerization time that accounts for the difference between the frequency of self-oscillations and the forcing resonance peak.

The correlation between Amp and $\mathrm{T}_{\mathrm{d}}$ is very robust. Responses from self-oscillating cells, cells stimulated with different stimulation strengths and different frequencies all lay on the same curve. This suggests that the Amp and $\mathrm{T}_{\mathrm{d}}$ are good measures to describe the underlying mechanism of depolymerization. The growth of Amp as the square of $T_{d}$, represents the core properties of actin depolymerization: first, as actin dissociates only from the pointed end of actin filaments, a longer time is required to disassemble more actin. Second, severing enhances the depolymerization rate by creating more pointed ends of actin filaments. As the bigger Amp comes from the more branched network, the role of severing becomes more significant at higher Amp. 
Experiments with cells simultaneously labeled LimE and Arp2/3 provided us the information about how Amp varies with the amount of activated Arp2/3. A further evidence to support the hypothesis would be the verification of the capping protein as the major factor determining $\mathrm{T}_{\mathrm{p}}$. Experiments comparing $\mathrm{T}_{\mathrm{p}}$ of normal cells and cells lacking capping protein can strongly support the hypothesis. However, deleting capping protein is lethal to cells. Although experiments with cells with simultaneously labeled LimE and capping protein to see the correlation between the peak of capping protein in the cortex and actin filaments in response to stimulation would provide similar information as what was done before [127], there is no evidence yet that oscillations of actin in self-oscillating cells is also controlled by the binding of capping protein.

In conclusion, the regulation of polymerization and depolymerization controls not only the strength but also the frequency of actin dynamics. Moreover, even for cAMP stimulation responses involving a much more complicated cascade of enzyme reactions, the underlying mechanism of the self-organization of actin dynamics is the same in the presence and absence of stimuli. 


\section{CHAPTER 5 \\ Myosin II sets the optimal response time scale}

\subsection{Motivation}

So far we have described actin dynamics in the absence of external stimulation (chapter 3) as well as how cAMP triggers the actin responses (chapter 4). We also investigated and proposed the leading roles of the associated proteins involved in the processes of polymerization and depolymerization of actin filaments. Besides actin binding proteins, the motor protein myosin II also binds to filamentous actin and is indispensible for maintaining the normal functioning of cell movement and development. By walking unidirectionally towards the barbed end (usually designated as the plus end.) of actin filaments, myosin II bundles enable contraction. Cells lacking myosin II show reduced stiffness and decreased cortical tension, they diminish their translocation speed and terminate cell division in suspension [72, 144-146]. Despite the significance of myosin II, whether myosin II is required for the rapid oscillations of actin cytoskeleton has not yet been studied.

Here we simultaneously observed the dynamics of myosin II and LimE in $D$. discoideum cells by co-expressing myosin II-GFP and LimE-mRFP to investigate the coupling between the dynamics of myosin II and actin in the absence of stimulation. Considering the fact that myosin II is functionally active only as filaments, the lack of heavy chain inhibits the entire function of myosin II $[61,69]$. To elucidate the role of myosin II in self-oscillations, we examined the dynamics of LimE in cells without myosin II heavy chains (which, from here on will be referenced to as myosin II-null or myosin II knock-out cells). We further explored the interplay between actin and myosin II in the presence of stimulation. Besides the actin dynamics itself, we also inspected how the interplay between actin and myosin II influences the cell morphology, especially the cringing phenomena in response to cAMP [95]. 


\subsection{Myosin II dynamics in the absence of external stimulation}

Cells simultaneously labeled myosin II-GFP and LimE-mRFP showing various types of oscillations in the absence of external stimulation: regular oscillations of both myosin II and LimE, regular oscillations only in myosin II but not in LimE, and regular oscillations only in LimE but not in myosin II, and also neither myosin II nor LimE oscillating. The occurrence of LimE and myosin II oscillate together (1489 oscillations observed from 387 cells) is higher than only LimE (764 oscillations observed from 387 cells) or myosin II (604 oscillations observed from 387 cells) oscillates. We further examined the oscillation period distributions for the cases mentioned above. Histograms are indistinguishable in all cases (Figure 5.1), implying that the oscillations of myosin II and LimE are independent of each other. Moreover, although it has been found that LimE-mRFP and LimE-GFP localized at different positions of the cell cortex [147], the distribution of oscillation periods did not change at different LimE labels (Figure 5.1A and Figure 3.5).
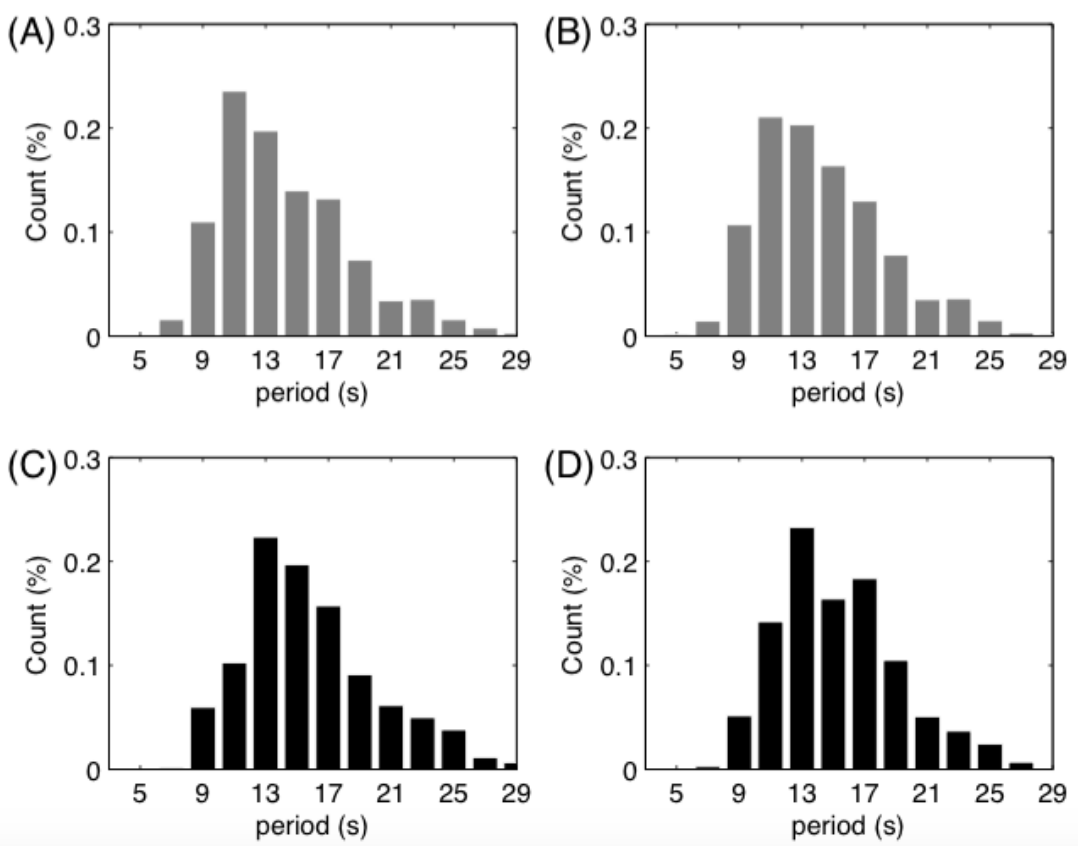

Figure 5.1: Oscillations of myosin II and LimE were independent of each other. 387 cells labeled both myosin II-GFP and LimE-mRFP were observed. Histograms of the oscillation period of (A) LimE in cells that only show oscillations in LimE $(\mathrm{N}=764)$. (B) LimE in cells showing oscillations in both LimE and myosin II $(\mathrm{N}=1489)$. (C) Myosin II in cells that only show oscillations in myosin II $(\mathrm{N}=604)$. (D) Myosin II in cells showing oscillations in both LimE and myosin II $(\mathrm{N}=1489)$. 
We further confirmed that myosin II is not necessary for self-oscillations by looking at the oscillation period of myosin II knock-out cells. Cells without myosin II showed comparable oscillation periods of LimE as found in wild-type cells (Figure 5.2A and Figure 3.5). Moreover, the occurrence as well as the duration of self-oscillations are also indepedent of myosin II (Figure 5.2B to 5.2D and Figure 3.7).
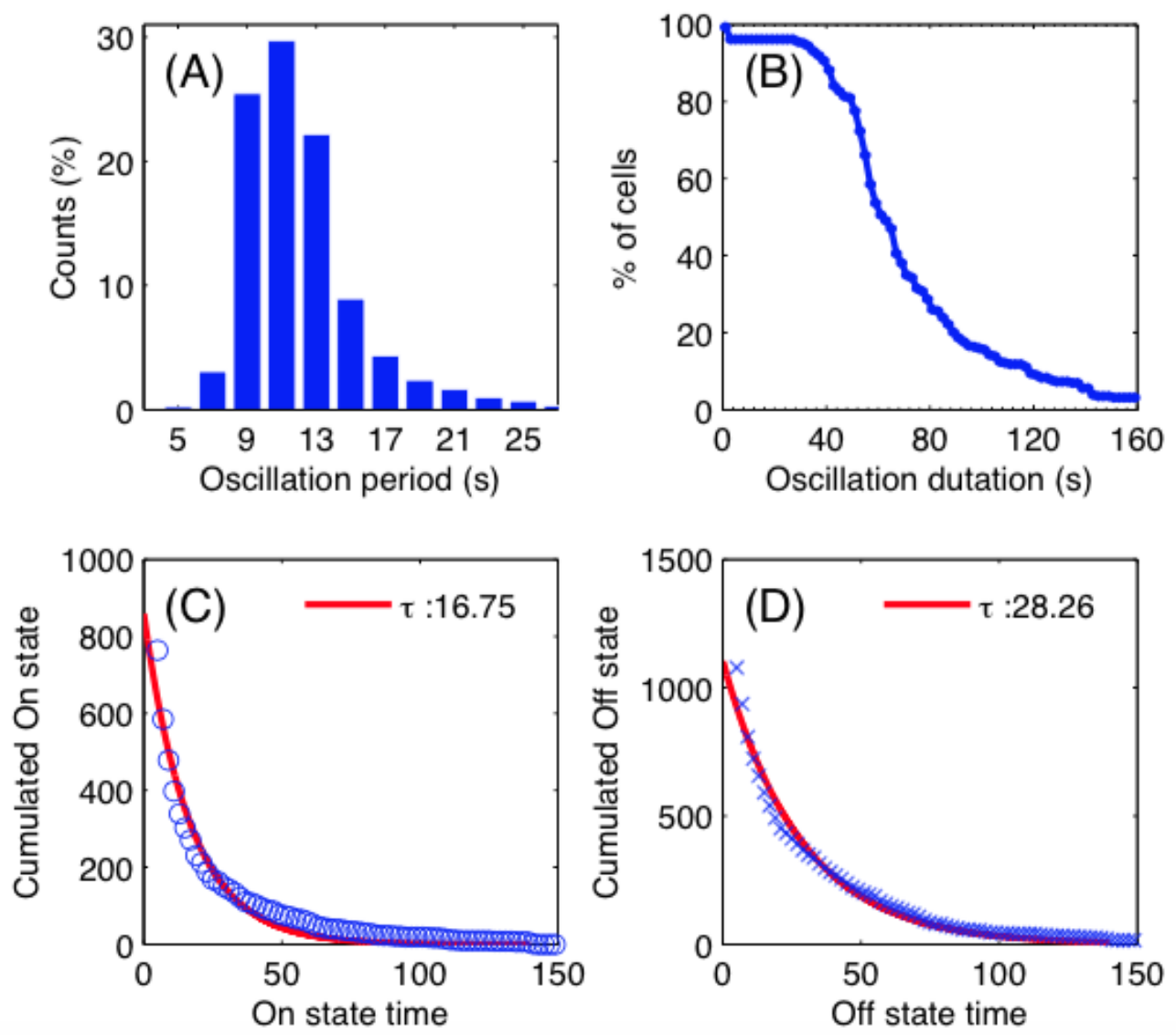

Figure 5.2: Properties of self-oscillations in LimE-GFP labeled myosin II-null cells. (A) Distribution of self-oscillation periods ( $\mathrm{N}=655)$. (B)-(D) 224 myosin II-null cells labeled with LimE-GFP were observed for $160 \mathrm{~s}$ in the absence of external stimulation. (B) Distribution of the longest time for which a cell can oscillate. (C) Distribution and average life time of oscillating and non-oscillating states. Circles display experimental data and the red line is the fit to a single exponential decay $\left(y=a \exp ^{-b x}, \tau=1 / b\right)$. (D) Distribution and average life time of non-oscillating states. Crosses are experimental data and the red line displays the fit to a single exponential decay ( $y=a \exp ^{-b x}, \tau=1 / b$ ) 


\subsection{The dynamics of myosin II in the presence of cAMP stimulation}

We next investigated the role of myosin II in cAMP responses by applying a single $1 \mathrm{~s}$ uniform stimulation of cAMP right in front of individual cells. To analyze how the dynamics of myosin II and actin influence each other, we observed cells co-expressing LimE-GFP and myosin II-mRFP. Although the fluorescence intensity of LimE-mRFP is much weaker than that of LimE-GFP, the global actin dynamics from both the labeled versions were indistinguishable.

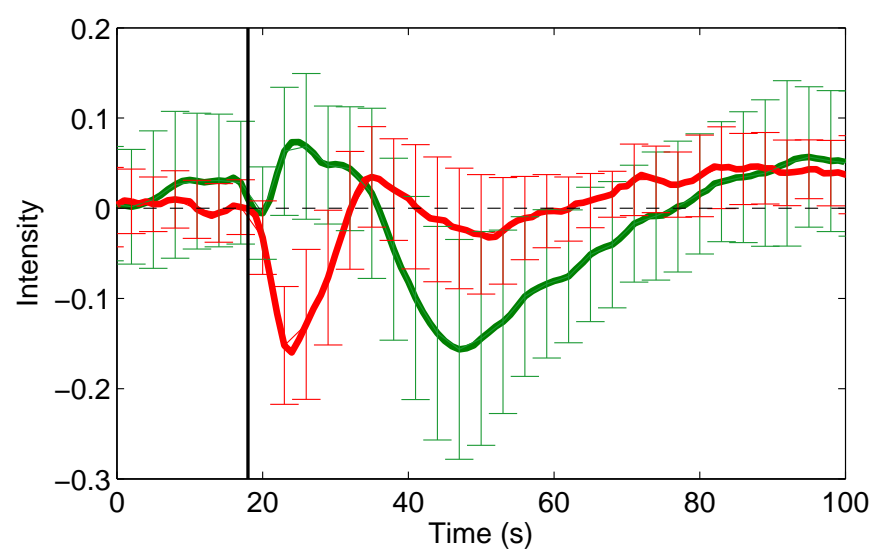

Figure 5.3: Responses of LimE and myosin II to a single $1 \mathrm{~s}$ cAMP. The stimulus was applied with $100 \%$ laser power to uncage $10 \mu \mathrm{M}$ BCMCM-caged cAMP. Dynamics of myosin II and LimE in response to a single $1 \mathrm{~s}$ stimulation. $42 \mathrm{D}$. discoideum cells labeled with myosin II-GFP and LimE-mRFP were observed. Red trace shows the average response from LimE-mRFP (the same as in (A)), whereas green trace shows the average response from myosin II-GFP. Errorbar shows the standard deviation. Vertical black line indicates the time cAMP was applied.

Upon stimulation by cAMP, abundant filamentous actin forms in the cortical region, resulting in a significant depletion of cytosolic LimE. The following excessive depolymerization of actin filament leads to a continuous increase of LimE in the cytosolic region. On the other hand, external stimulation seems to partially release myosin II from the cortex while actin filament grows there. About $15 \mathrm{~s}$ after stimulation, myosin II gradually accumulates in the cortical region. Compared to actin dynamics, the translocation of myosin II to the cortex is much slower but more persistent (Figure 5.3). Etzrodt et al. has reported that myosin II requires more than $20 \mathrm{~s}$ for maximal accumulation in the cortical region, which is consistent with our results. However, they did not observe a release of myosin II after stimulation. 
It is known that in the presence of a chemical gradient, myosin II localizes in the rear of the cell. Moreover, myosin II can rapidly redistribute according to the direction of the gradient, involving a rapid release from the cortical region (Figure 5.4B). But a release of myosin II from the cortex in response to a uniform stimulus of cAMP has not been reported. From the increase and the subsequent decrease in the amount of myosin II observed in our experiments (Figure 5.3), it is possible that the release of myosin II in response to a uniform stimulation of cAMP was overlooked so far (Figure 5.4A).

(A)
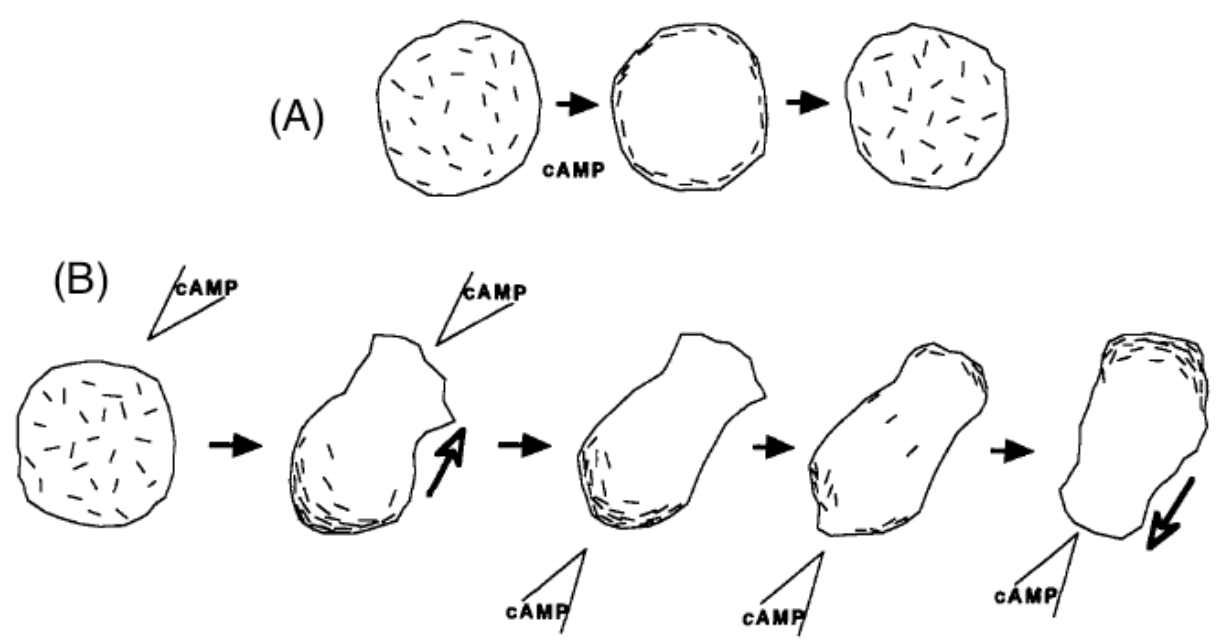

Figure 5.4: Schematic diagram showing myosin II of $D$. discoideum cells changes its localization in response to cAMP. (A) In response to a uniform stimulation, myosin II is translocated isotropically around the cortex. (B) Myosin II always localizes to the rear part of the cell on a local stimulation with a microcapillary ( $\nearrow$ shows the direction of cell movement). Upon changing the direction of the stimuli, myosin II is released from the original rear-end of the cortex and is translocated to the new position opposite to where the stimulation is applied $(\swarrow$ shows the direction of cell movement under the new gradient). The figure is adapted from [148].

We next verified if a uniform stimulation of cAMP can also trigger the release of myosin II from the cortex but the main challenge is that the signal of the released myosin II is too weak to be distinguished from noise. Our goal is thus to enhance the amount of cortical myosin II. Considering the fact that myosin II starts to translocate to the cortex around $15 \mathrm{~s}$ after stimulation by cAMP and starts to disassemble and return to the cytosol around $25 \mathrm{~s}$ after stimulation, the application of another stimulation about 10-25 s after the first stimulation can achieve our goal without any genetic modification of the cell. The periodic increases of myosin II-GFP signal 
confirms the rapid release of myosin II under the stimulation of uniform cAMP (Figure 5.5). As filamentous actin rapidly forms after the stimulation of cAMP, the corresponding signal of LimE-mRFP in the same cells decreases instead.
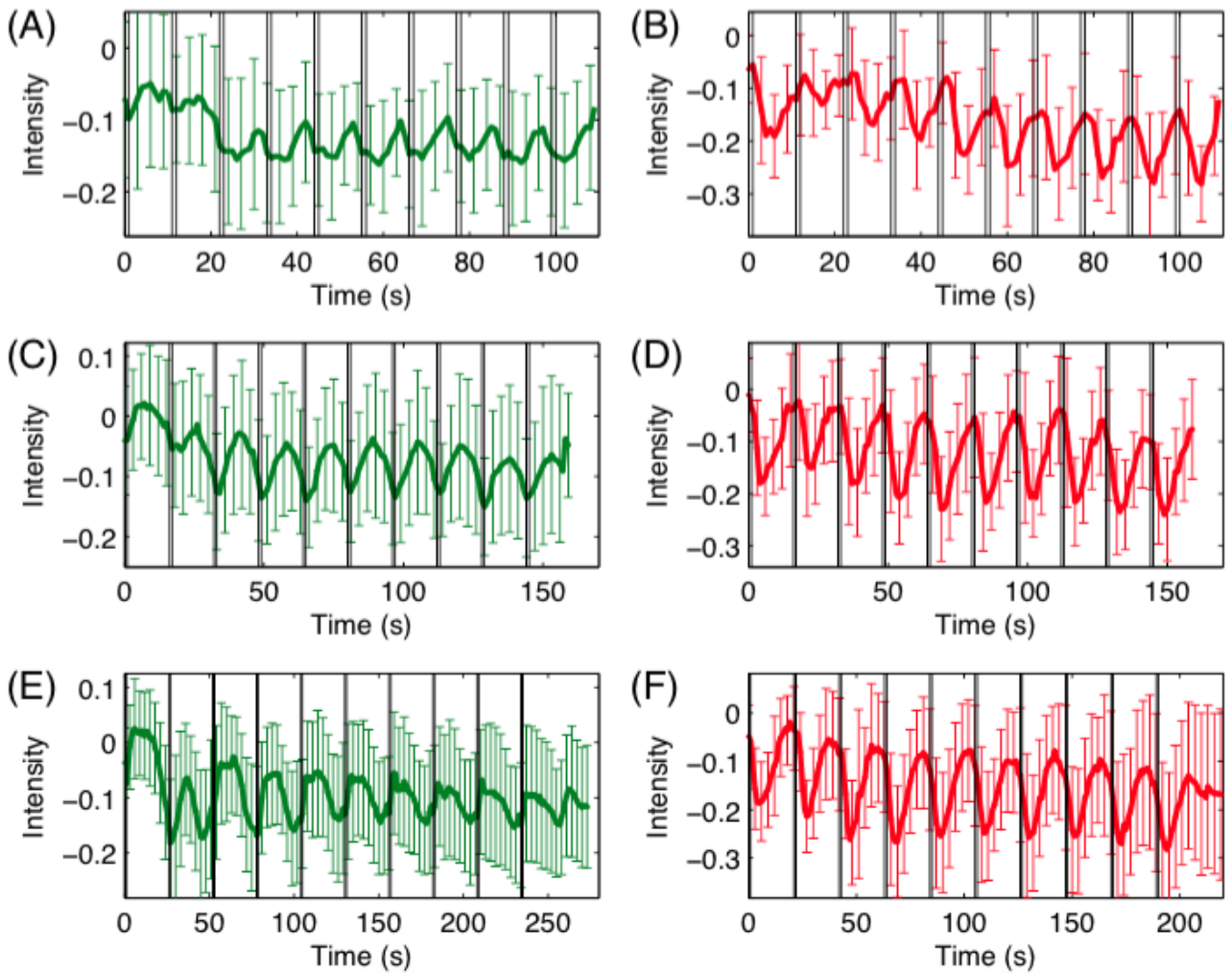

Figure 5.5: Periodic stimuli were applied to verify the release of myosin II right after stimulation. Ten $1 \mathrm{~s}$ stimuli of cAMP were applied to the individual cells. Stimuli were applied with $100 \%$ laser power to uncage $10 \mu \mathrm{M}$ BCMCM-caged cAMP. Signal from cells co-expressing myosin II-GFP and LimE-mRFP was collected. (A), (C) and (E) show the average responses of myosin II-GFP to periodic stimuli with intervals of $11 \mathrm{~s}, 16 \mathrm{~s}$ and $26 \mathrm{~s}$, respectively. (B),(D) and (F) show the average responses of LimE-mRFP to periodic stimuli with intervals of $11 \mathrm{~s}, 16 \mathrm{~s}$ and $21 \mathrm{~s}$, respectively. The average signals were from 15 cells in (A) \& (B), 23 cells in (C) \& (D), 28 cells in (E) and 27 cells in (F). Vertical black lines indicate the time cAMP was applied. Error bar shows the standard deviation. For reasons of visibility only every third error bar is displayed. 


\subsection{Myosin II helps the recovery of cortical actin filaments.}

To investigate how myosin II affects cAMP responses, we first applied a single $1 \mathrm{~s}$ stimulus to the myosin II-null cells. Comparison of the LimE signals from the wildtype and the myosin II knock-out cells shows that the difference mainly occurred after the peak of the signal (Figure 5.6A). The delayed recovery of LimE suggests a slower response time scale for cells lacking myosin II. However, it is very difficult to determine the time to finish one oscillation after stimulation precisely. Considering the fact that the response amplitude is maximal when the intrinsic frequency matches the frequency of the external stimulation, the best way to capture the oscillation period is through the resonance curves. Periodic stimuli were applied to the myosin IInull cells using the same stimulation as for wild-type cells (black curve in Figure 4.4) to prevent a resonance shift due to varying stimulation strength. Myosin II-null cells show a clear resonance peak at a forcing period of $36 \mathrm{~s}$ (Figure 5.6B), which is significantly delayed as compared to the resonance at $24 \mathrm{~s}$ found for the wild-type cells (black curve in Figure 4.4), suggesting an important role for myosin II in the recovery of cortical actin filaments.
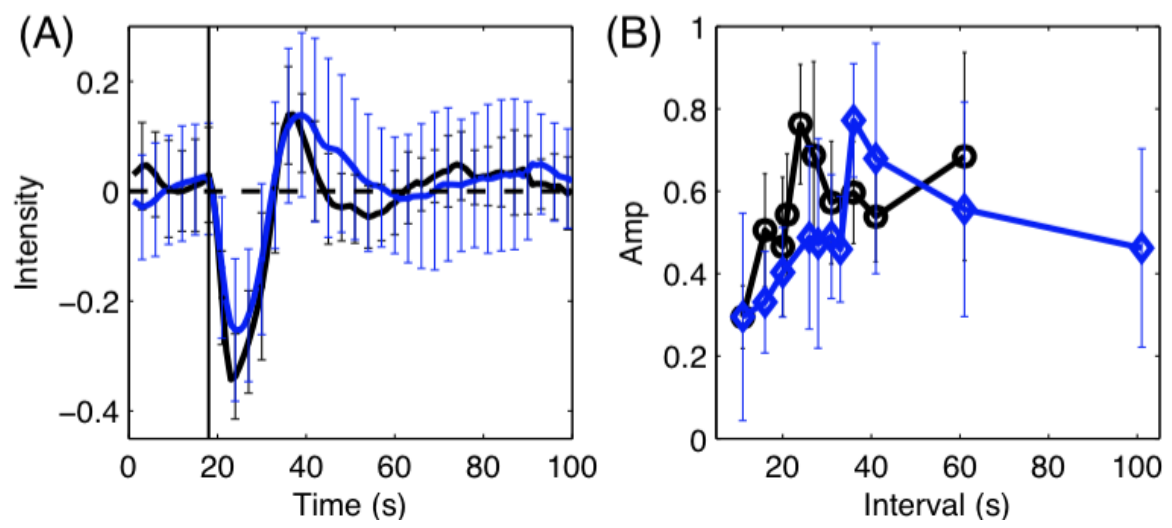

Figure 5.6: Comparison of LimE dynamics in wild-type (black) and myosin II-null (blue) cells. (A) Dynamics of LimE-GFP in myosin II-null $(\mathrm{N}=56)$ as well as wild-type $(\mathrm{N}=31)$ cells in response to a single $1 \mathrm{~s}$ stimulation. The stimulus was applied with $100 \%$ laser power to uncage $10 \mu \mathrm{M}$ BCMCM-caged cAMP. Error bar shows standard deviation. Vertical black line indicates the time cAMP was applied. (B) Myosin II-null cells show a resonance peak shift. All Myosin II-null cells and wild-type cells were stimulated with $10 \mu \mathrm{M}$ BCMCM-caged cAMP. At the specified interval, 10 stimuli were applied to each cell and we analyzed the amplitude of individual responsive traces. At least 10 cells were observed for every interval. Black line shows the resonance curve from wild-type cell (the same as Figure 4.4). Symbols show the stimulation intervals done in the experiment and the error bar shows the standard deviation. 
As myosin II can bind on actin filaments to form actomyosin complex, we proposed that the interaction between actin and myosin II not only enables the translocation of myosin II from cytosol to cortex, but also accelerates the accumulation of filamentous actin in the cortical region. It is known that the translocation of myosin II to the cortex requires actin filaments. Cells treated with Latrunculin A are not only incapable of assembling actin filaments but are also unable to accumulate myosin II in the cortex $[149,150]$. The average response to a $1 \mathrm{~s}$ stimulation also shows that right after the excessive disassembly of actin filaments, the fluorescence signal of myosin II in the cytosolic region decreases along with LimE (Figure 5.5B), suggesting a coupling between the dynamics of filamentous actin and myosin II. To further support our hypothesis, we analyzed the time at which LimE starts to translocate to the cortex after the excessive disassembly of cortical filamentous actin (i.e., $\mathrm{T}_{\mathrm{p}}+\mathrm{T}_{\mathrm{d}}$ of LimE, as defined in Figure 4.1) and the time myosin II needs to stop its accumulation in the cortex (i.e., Tp of myosin II, as defined in Figure 4.1) from individual cells. In spite of the diverse response times among cells due to cell-to-cell variability (section 4.4), the time myosin II stop its accumulation in the cortex (i.e., Tp of myosin II) was always correlated with the time LimE starts to re-translocate to cortex (i.e., $T_{p}+T_{d}$ of LimE, Figure 5.7).

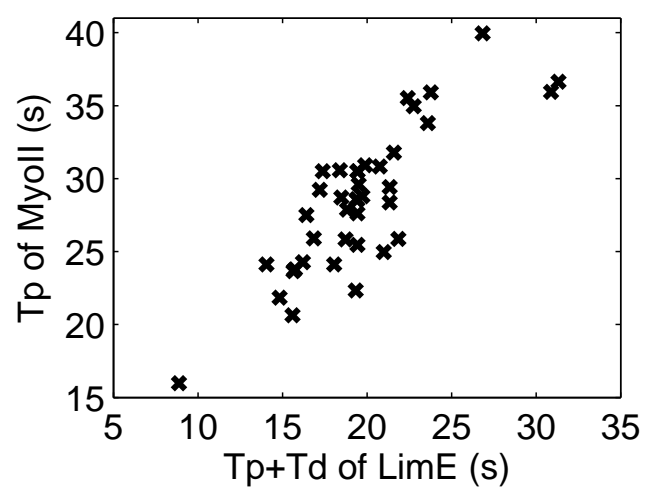

Figure 5.7: Translocation of myosin II to the cortex relies on the dynamics of the actin filaments. 40 cells simultaneously labeled with myosin II-GFP and LimE-mRFP were observed. Y-axis shows ( $T_{p}$ of MyoII) the time the cytosolic signal of myosin II-GFP needed to reach the minimum after stimulation and $\mathrm{x}$-axis $\left(\mathrm{T}_{\mathrm{p}}+\mathrm{T}_{\mathrm{d}}\right.$ of LimE) shows the time the cytosolic signal of LimE-mRFP needed to reach maximum after stimulation. $T_{p}$ and $T_{d}$ are defined as shown in Figure 4.1. Each cross shows the corresponding responses of LimE and myosin II from one cell. 
The couple dynamics between myosin II and filamentous actin implying the formation of actomyosin structures that allows cells to contract. It is known that within $3 \mathrm{~s}$ of a brief stimulus of cAMP, $D$. discoideum cells change shape rapidly, slow down and then cease random motion for about $20 \mathrm{~s}$, a response referred to as cringing [95, 150153]. The shape variation also leads to a change in the amount of light scattered, which has been extensively used as an indication of the passing of cAMP waves in pattern formation studies during the early aggregation of $D$. discoideum [8-13]. Although myosin II is essential for cell contraction, the cringe response has been observed not only in wild-type cells but also in myosin II knock-out cells [145, 154].

To investigate the mechanism of contraction and the role of myosin II during this process, we next analyzed the morphology of the adherent cells in the presence and absence of myosin II. When we compare the fluorescence image (showing the distribution of LimE) and the bright field image of a cell, the area occupied by the fluorescent LimE is similar to the area occupied by the cell in the bright field image. We thus regarded the area occupied by the fluorescent LimE signal as the actual projected cell area. Figure 5.8 shows that for wild-type cells, upon stimulation with cAMP, the area first increased and reached a maximum around $12 \mathrm{~s}$ after the stimulation. A sharp decrease in area followed, which reached a minimum around $35 \mathrm{~s}$ after stimulation and finally returned to the basal area $60 \mathrm{~s}$ after the stimulation. For myosin II-null cells, stimulation induced not only a slower expansion in the area but also a much slower size recovery to the original morphology (blue line in Figure 5.8).

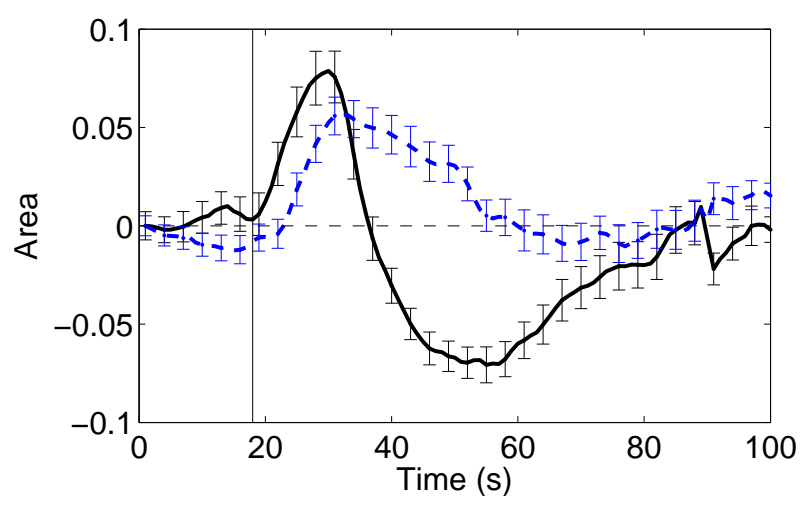

Figure 5.8: Myosin II-null cells cannot contract after stimulation by cAMP. Cell sizes were observed in myosin II-null cells (blue, $\mathrm{N}=31$ ) and wild-type cells (black, $\mathrm{N}=25$ ) after stimulation. The area is defined by normalizing the size of individual cells to its own average size before stimulation. Error bar shows the standard error (SE). 
The rapid growth of filamentous actin everywhere around the cortex pushes the membrane to instigates the increase of area as well as transforms the irregular cell shape into a circular morphology, resulting in a reduction of scattered light [155]. Since myosin II-null cells also grow actin filament in the cortical region in response to stimulation (blue trace in Figure 5.6A), a morphology change and thus a reduction in the scattered light can still be observed without myosin II. It is worth to note that myosin II-null cells show a delay in the expansion of area (blue trace in Figure 5.8). Considering the fact that the cross-linking of myosin II stablizes the actin cortex [72], myosin II-null cells lose their cortical integrity and thus may expand their membrane less effectively.

\subsection{The role of myosin II before its translocation to the cortex}

So far, the main differences happened around the time myosin II translocated to the cortical region. To investigate if the cortical release of myosin II additionally influences the actin dynamics, we dissected the time before the translocation of myosin II to the cortex into two parts: $\mathrm{T}_{\mathrm{p}}$ (time for which polymerization dominates, as defined in Figure 4.1), $\mathrm{T}_{\mathrm{d}}$ (time when depolymerization dominates, as defined in Figure 4.1). The amplitude (Amp) defines the difference between the maximum and minimum of cytosolic signal was investigated to know the amount of depolymerized actin as well. Wild-type and myosin II-null cells show comparable distribution of $\mathrm{T}_{\mathrm{p}}$ (Figure 5.9A \& 5.9B), suggesting a resemblance of actin dynamics during polymerization. As more myosin II-null cells distributed in the long depolymerization times than wild-type cells (Figure 5.9D \& 5.9E), we further plotted Amp against $T_{d}$ to verify if the broader distribution implying a different depolymerization mechanism or due to larger cell-to-cell variability. We found that data from wild-type and myosin IInull cells lay on the same curve. The logarithmic fitting also shows comparable results in myosin II-null cells (Figure 5.10B, $\mathrm{T}_{\mathrm{d}}$ grows with $\mathrm{Amp}^{0.56}$ ) and in wild-type cells (Figure 4.11, $\mathrm{T}_{\mathrm{d}}$ grows as $A m \mathrm{p}^{0.50}$ ). The plot of Amp against $\mathrm{T}_{\mathrm{p}}$ was also examined and all responses from wild-type and myosin II-null cells again lay on the same curve, suggesting a similar underlying mechanism controlling the dynamics of filamentous actin even in the absence of myosin II: the length of the polymerization dominated time is independent of stimulation strength (Figure 5.9C) but more filamentous actin (larger Amp) requires a longer depolymerization time (Figure 5.9F). 

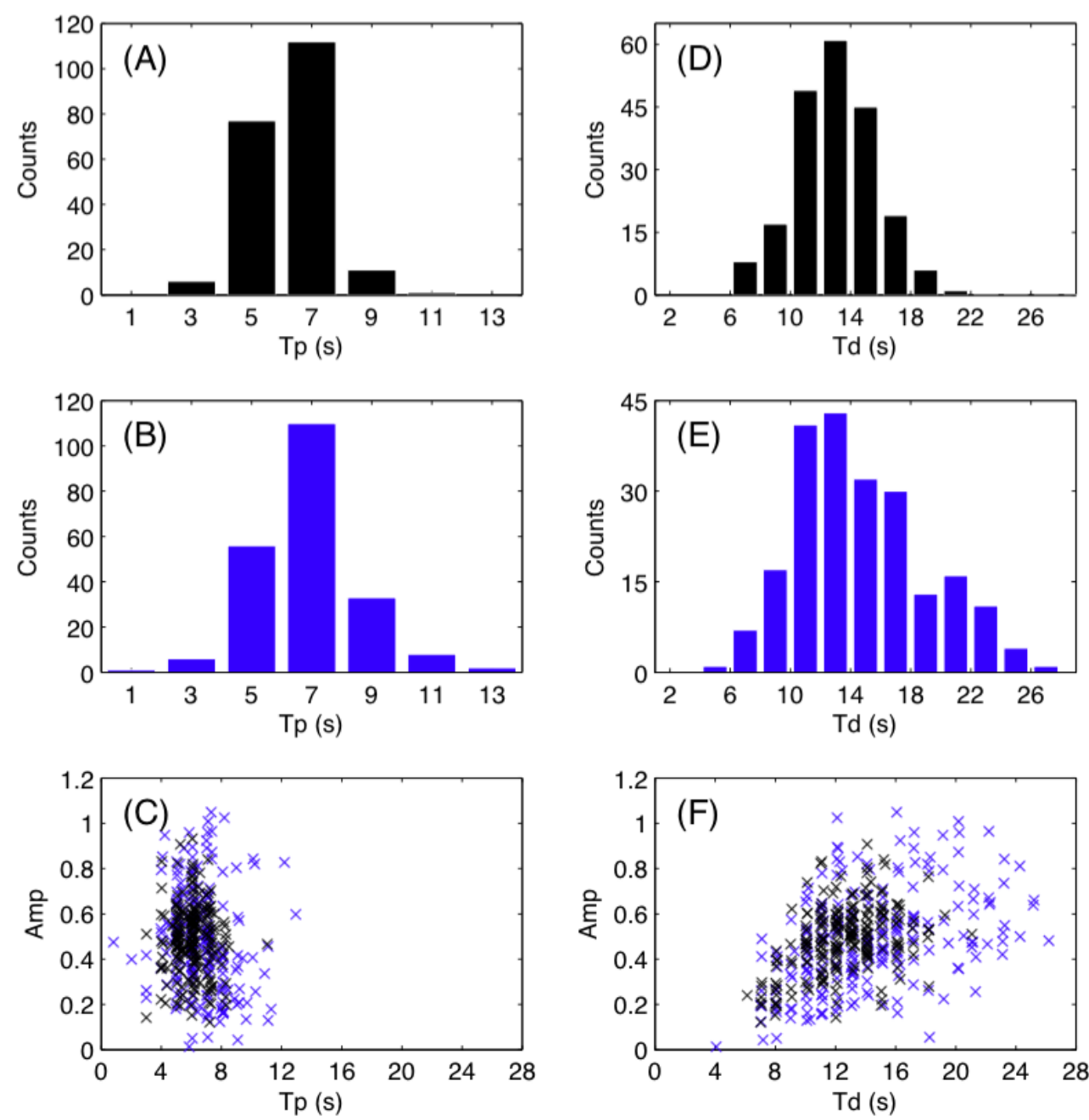

Figure 5.9: Effect of myosin II on filamentous actin dynamics before its translocation to the cortex. Comparison of $\mathrm{T}_{\mathrm{p}}$ and $\mathrm{T}_{\mathrm{d}}$ in wild-type (black) and myosin II-null cells (blue). (A) Histogram of $T_{p}$ in wild-type cells $(N=207)$. (B) Histogram of $T_{p}$ in myosin II-null cells $(\mathrm{N}=216)$. (C) Amp vs. $\mathrm{T}_{\mathrm{p}}$ in wild-type and myosin II-null cells. Each symbol shows the response from one cell. (D) Histogram of $T_{d}$ in wildtype cells $(N=207)$. (E) Histogram of $T_{p}$ in wild-type cells $(N=216)$. (F) Amp vs. $T_{d}$ in wild-type and myosin II-null cells. Each cross shows the responses of one cell to a single $1 \mathrm{~s}$ stimulus with different concentrations, which was applied with $100 \%$ laser power to uncage $100 \mathrm{nM}$ to $100 \mu \mathrm{M}$ BCMCM-caged cAMP. 


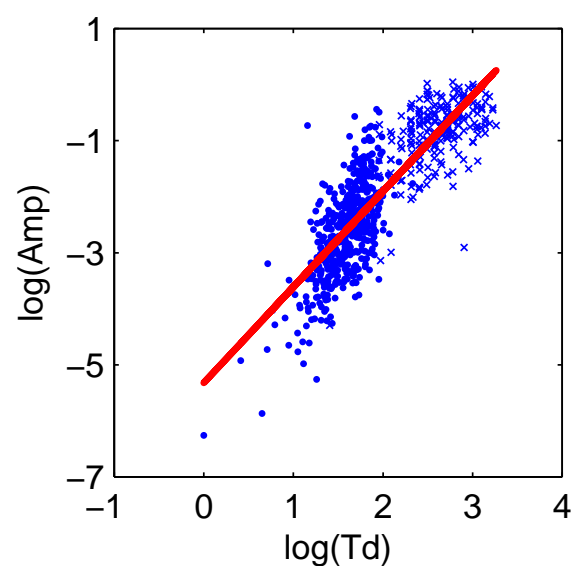

Figure 5.10: Logarithm fitting of relation between Amp and $T_{d}$. Myosin II-null cells labeled LimE-GFP were observed. Dots show the analysis from 502 self-oscillating cells and crosses show the analysis from 232 cells responding to one $1 \mathrm{~s}$ stimulus. The stimulus was applied with $100 \%$ laser power to uncage different concentrations of BCMCM-caged cAMP (from $30 \mathrm{nM}$ to $100 \mu \mathrm{M}$ ). Red line shows: $\log (\mathrm{Amp})=-5.4+1.78 \times \log \left(\mathrm{T}_{\mathrm{d}}\right)$.

The data from myosin II-null cells is scattered over a larger area than the wildtype cells, suggesting a substantial cell-to-cell variability in myosin II-null cells (Figure 5.9C \& 5.9F). To inspect the origin of the significant variations among myosin II-null cells, we first examined how different starvation times change the properties of Amp and $\mathrm{T}_{\mathrm{d}}$. Self-oscillating cells scattered more as they starved longer than 9 hours (Figure 5.11B). However, cells starved between 6 and 13 hours seem to show similar scattering in Amp and $\mathrm{T}_{\mathrm{d}}$ in response to stimulation (Figure 5.11A).

Due the fact that cells lacking myosin II have a disturbed cell division in suspension, the sizes of myosin II-null cells are in general much bigger (as shown in histograms in Figure 5.12) [156-159]. However, myosin II-null cells can divide through an adhesion dependent mechanism once they are on a solid surface [160-163]. Here we looked at the wild-type and myosin II-null cells to see how they change their sizes during the experimental time on the solid surface (Figure 5.12): myosin II-null cells were much larger than wild-type cells. Some myosin II-null cells tend to decrease their sizes with extended measurement time on the solid surface but the majority of myosin II-null cells did not change their size during the experiment, just like wild-type cells. Detailed analysis of Amp, $T_{d}$ and $T_{p}$ reveals that dynamics of filamentous actin is independent of the size of the cells (Figure 5.13). 

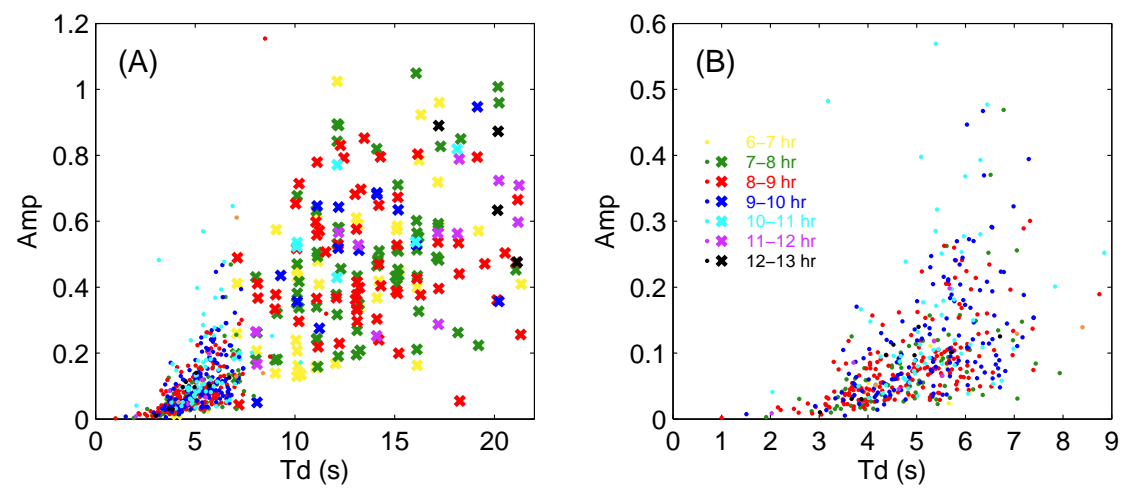

Figure 5.11: Effect of starvation time on actin dynamics in myosin II-null cells $(N=734)$. Amp and $T_{d}$ of cytosolic signals were analyzed. Dots show the analysis of self-oscillating cells $(\mathrm{N}=502)$. Crosses show the analysis of cells responding to a single $1 \mathrm{~s}$ stimulus $(\mathrm{N}=232)$. The stimulus was applied with $100 \%$ laser power to uncage BCMCM-caged cAMP with concentration ranging from $100 \mathrm{nM}$ to $100 \mu \mathrm{M}$. (A) Different colors show the data from different starvation times. (B) A magnification of (A) to see the data of self-oscillating cells more clearly.
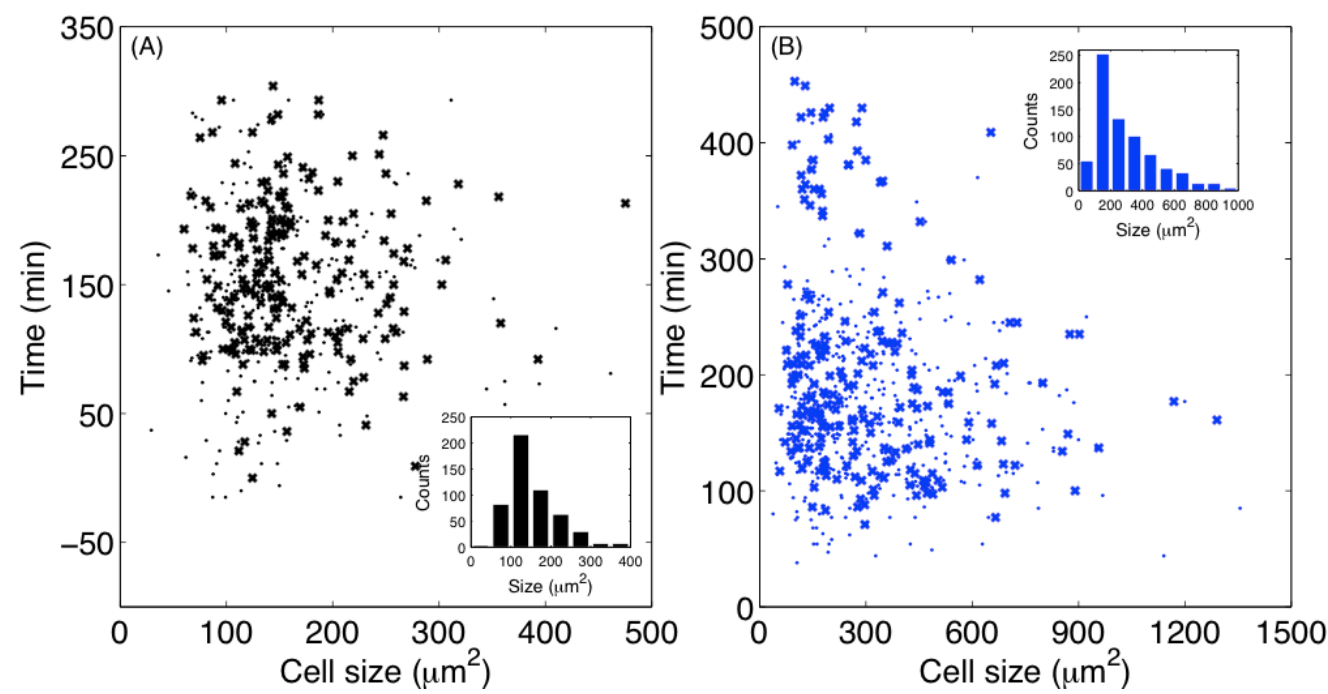

Figure 5.12: Correlation analysis between cell size and the time cells stay on the solid surface. Sizes were observed in wild-type cells (A) and myosin II-null cells (B). Inset shows the histogram of the sizes from all corresponding cells. Dots show the analysis of self-oscillating cells. Crosses show the analysis of cells responding to a single $1 \mathrm{~s}$ stimulus. The stimulus was applied with 100\% laser power to uncage BCMCM-caged cAMP with concentration ranging from $100 \mathrm{nM}$ to $100 \mu \mathrm{M}$. Each symbol shows the size of one cell at the corresponding time. Blue dots, $\mathrm{N}=502$; blue crosses, $\mathrm{N}=232$; black dots, $\mathrm{N}=300$; black crosses, $\mathrm{N}=231$. 

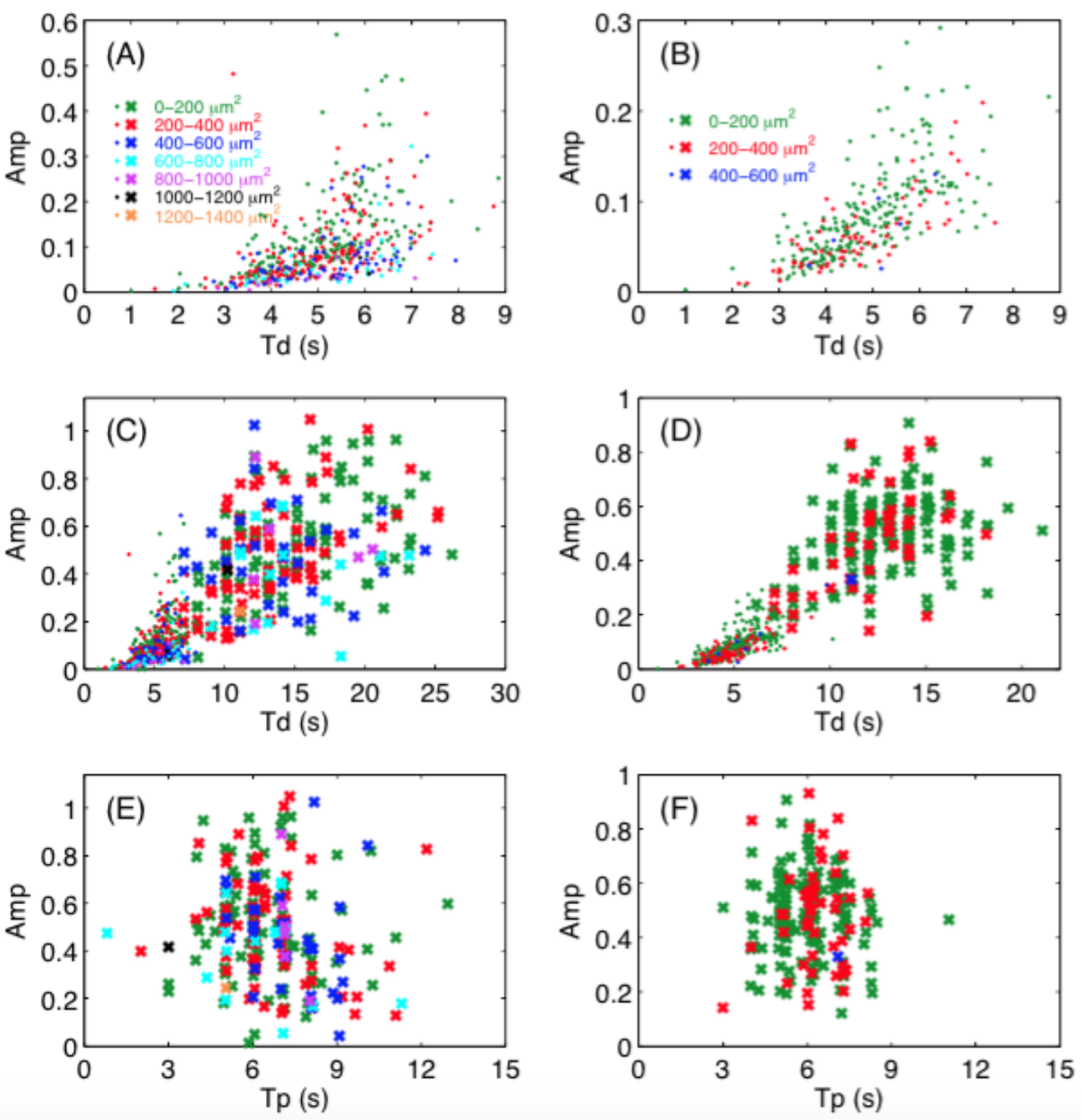

Figure 5.13: Effect of cell sizes on actin dynamics in myosin II-null $(N=734$, A,C,E. Sizes shown in the legend apply also to (C) and (E).) and wild-type cells ( $N=531$, B,D,F. Sizes shown in the legend apply also to (D) and (F).). Amp, $T_{p}$ and $\mathrm{T}_{\mathrm{d}}$ were defined as Figure 4.1. Dots show the analysis of self-oscillating cells. Crosses show the analysis of cells responding to a single $1 \mathrm{~s}$ stimulus. The stimulus was applied with $100 \%$ laser power to uncage BCMCM-caged cAMP with concentration ranged from $100 \mathrm{nM}$ to $100 \mu \mathrm{M}$. (A)\&(B) Amp vs. $\mathrm{T}_{\mathrm{d}}$ in self-oscillating cells. (C)\&(D) Amp vs. $\mathrm{T}_{\mathrm{d}}$ in self-oscillating cells as well as cells responding to stimulation. (E)\&(F) Amp vs. $T_{p}$ in cells responding to stimulation. 


\subsection{Conclusion}

Here we investigated the role of myosin II in rapid oscillations of the cytoskeletal network in the absence of external stimulation. We found no correlation between the self-oscillations of actin and any observable dynamics of myosin II or even its presence, suggesting that myosin II is not necessary for the rapid oscillations in the cytoskeletal networks.

However, immediately after the stimulation of cAMP, actin filaments grow in the cortical region whereas myosin II is released from the cortex (out-of-phase signals). As myosin II is indispensable for inhibiting the formation of pseudopods [72, 144-146], the release of myosin II enables the freshly formed filamentous actin to induce membrane protrusions. Most proteins in the signaling pathway stimulation or regulating actin network are translocated to the cortex within $10 \mathrm{~s}$ after the stimulation of cAMP (such as PHcrac, Ras, Aip1, Coronin, etc.) [109, 110, 140, 164]. In contrast, previous studies claimed that myosin II responded to the stimulation in a much later time scale (around $30 \mathrm{~s}$ after stimulation) [165]. Our finding points out that myosin II is in fact responsive, though less pronounced, within $10 \mathrm{~s}$, but is released from the cortex into the cytosol (Figure 5.5).

The later translocation of myosin II to the cortical region ( $30 \mathrm{~s} \mathrm{cf.} 10 \mathrm{~s}$ ) is due to the fact that the translocation of myosin II requires filamentous actin. We confirmed the coupling between the dynamics of myosin II and LimE during their translocation to the cortical region (Figure 5.7). The delay in the recovery of cortical filamentous actin in myosin II-null cells (Figure 5.6A) suggests that the interaction between myosin II and actin filaments not only carries myosin II from the cytosol to the cortex but also enhances the efficiency of actin accumulation in the cortical region.

The main difference between the morphology of wild-type and myosin II mutant cells is also found during the time that myosin II and filamentous actin translocate to the cortex together: wild-type cells rapidly shrink their size to $10 \%$ smaller of their size before stimualtion and then gradually recover, whereas myosin II-null cells slowly returned to their original sizes (Figure 5.8), suggesting that they lose the property to contract rapidly. Considering the fact that myosin II walking on actin filaments enables contraction, the accumulation of actomyosin filaments in the cortex thus lead to a significant decrease in the imaged area. Cells without myosin II decreases their size much more slowly, implying the indispensible role of myosin II in maintaining cell morphology. 


\section{Conclusion and Outlook}

In this work, we investigated the actin network dynamics of $D$. discoideum in the absence and presence of external stimulation of cAMP. We quantified first the selfoscillations: the actin networks of cells oscillate with a period around $12 \mathrm{~s}$ and this property is independent of the passage number, starvation time, cell sizes and even regulatory proteins like myosin II. We discovered that self-oscillations originate from the global synchronization of actin polymerization and depolymerization in the cell cortex and can be observed in all cells. The oscillation periods are controlled by the binding time of the capping protein: as filamentous actin shows little branching owing to the small amount of activated Arp2/3 (branching protein), the binding time of capping protein determines the number of polymerized actin filaments. Since the following depolymerization only happens from the pointed end of actin filaments, it takes longer to depolymerize more actin filaments. Considering the fact that one oscillation period includes both the polymerization and depolymerization process, the binding time of capping protein therefore controls the oscillation period through determining the amount of polymerized filamentous actin.

It is the synchronizations that enable us to learn the frequency of the intrinsic cytoskeletal dynamics. However, cells spend more time in the non-oscillatory state and it is still unknown what initiates the global synchronization. Since it has been found in the actin dynamics of cancer cells that calcium is responsible for oscillations with periods of around $30 \mathrm{~s}$ [122], examination of the influence of calcium on self-oscillations should be a good start.

Second, we investigated the effect of stimulation strength on actin dynamics. We found that the response amplitude grows with stimulation strength only in a very narrow region of stimulation, after which the response amplitude reaches a plateau. This threshold varies a lot among cells. Moreover, the response amplitudes are scattered not only with respect to different stimulation strength at the plateau but also to the same stimulation. In spite of the large cell-to-cell variability, when plotting the single response amplitudes against the corresponding polymerization and depolymerization times, a nearly constant and quadratic relationship are visible, respectively, suggesting an universal underlying mechanism. 
It has been reported that actin patches can form randomly anywhere in the cell cortex $[166,167]$. Pseudopods grow from the regions with the highest density of actin patches [132]. Cells have a basal cycle for the extension of pseudopod, which is a non-random probabilistic process $[103,107]$. Shallow gradients does not change the dynamics of patch formation but can bias the probabilities of patch formation to enhance the density of patches aligned with the direction of the gradient $[102,105]$. This corroborates our finding that the response upon external stimulation can be well described by the same mechanism and components of self-oscillations, while the larger response amplitude are ascribed to an increased amount of Arp2/3.

It is still unclear what the factor controlling the amount of activated Arp2/3 after stimulation is. Besides examining the dynamics of proteins involved in the signaling pathways upstream of Arp2/3, it is worthwhile to check whether genetic variations play a role on the differences among individuals. To uncover the underlying determinant, a new method that enables simultaneous stimulation and recording of a large number of cells is required for an efficient systematic screening.

Finally, we investigated the interplay between actin and myosin II. There is no correlation between the self-oscillations of actin and any observable dynamics of myosin II or even its presence. However, immediately after the stimulation of cAMP, actin filaments grow in the cortical region whereas myosin II is released from the cortex (out-of-phase signals). As myosin II inhibits the formation of pseudopods, the release of myosin II enables the freshly formed filamentous actin to induce membrane protrusions. Most proteins in the signaling pathway or regulating actin network are translocated to the cortex within $10 \mathrm{~s}$ after the stimulation of cAMP (such as PHcrac, Ras, Aip1, Coronin, etc.). In contrast, previous studies claimed that myosin II responded to the stimulation in a much later time scale (around $30 \mathrm{~s}$ after stimulation) [165]. Our finding points out that myosin II is in fact responsive, though less pronounced, within $10 \mathrm{~s}$, but is released from the cortex into the cytosol. The later translocation of myosin II to the cortical region (30 s cf. $10 \mathrm{~s}$ ) is due to the fact that the translocation of myosin II requires filamentous actin. The interaction between myosin II and actin filaments not only carries myosin II from the cytosol to the cortex but also enhances the efficiency of actin accumulation in the cortical region. The actomyosin complex further enables rapid cell contraction. Myosin II-null cells show a delay in the recovery of cortical actin filaments as well as incapability of contractions, revealing the essence of myosin II in regulating actin dynamics and maintaining cell morphology. 


\section{Bibliography}

[1] Ludwig Eichinger \& Francisco Rivero, editor. Dictyostelium discoideum protocols. Humana press, 2006.

[2] G. Potts. Zur physiologie des dictyostelium mucoroides. Flora (Jena), 1902.

[3] R. Sussman and M. Sussman. Physical charaterization of deoxyribonucleic acids in dictyostelium discoideum. Arch. Biochem. Biophys, 1971.

[4] J. Franke and R. Kessin. Adefined minimal medium for axenic strains of dictyostelium discoideum. Proc. Natl. Acad. Sci USA, 1977.

[5] R. L. Chisholm and R. A. Firtel. Insights into morphogenesis from a simple developmental system. Nat. Rev. Mol. Cell Biol., 5(7):531-541, July 2004.

[6] I. M. Zadahames and J. M. Ashworth. Cell-cycle during vegetative stage of dictyostelium-discoideum and its response to temperature-change. J. Cell Sci., 32(AUG):1-20, 1978.

[7] M. Clarke. Recent insights into host-pathogen interactions from dictyostelium. Cell. Microbiol., 12(3):283-291, March 2010.

[8] K. J. Tomchik and P. N. Devreotes. Adenosine-3',5'-monophosphate waves in dictyostelium-discoideum - a demonstration by isotope dilution-fluorography. Science, 212(4493):443-446, 1981.

[9] S. Sawai, P. A. Thomason, and E. C. Cox. An autoregulatory circuit for long-range self-organization in dictyostelium cell populations. Nature, 433(7023):323-326, January 2005.

[10] D. Dormann, J. Y. Kim, P. N. Devreotes, and C. J. Weijer. camp receptor affinity controls wave dynamics, geometry and morphogenesis in dictyostelium. $J$. Cell Sci., 114(13):2513-2523, July 2001.

[11] E. Ben-Jacob, I. Cohen, and H. Levine. Cooperative self-organization of microorganisms. Adv. Phys., 49(4):395-554, June 2000.

[12] P. N. Devreotes, M. J. Potel, and S. A. Mackay. Quantitative-analysis of cyclicamp waves mediating aggregation in dictyostelium-discoideum. Dev. Biol., 96(2):405-415, 1983. 
[13] E. Palsson, K. J. Lee, R. E. Goldstein, J. Franke, R. H. Kessin, and E. C. Cox. Selection for spiral waves in the social amoebae dictyostelium. Proc. Natl. Acad. Sci. U. S. A., 94(25):13719-13723, December 1997.

[14] C. J. Weijer, G. Duschl, and C. N. David. Dependence of cell-type proportioning and sorting on cell-cycle phase in dictyostelium-discoideum. J. Cell Sci., 70(AUG):133-145, 1984.

[15] M. Wang, R. J. Aerts, W. Spek, and P. Schaap. Cell-cycle phase in dictyosteliumdiscoideum is correlated with the expression of cyclic-amp production, detection, and degradation - involvement of cyclic-amp signaling in cell sorting. Dev. Biol., 125(2):410-416, February 1988.

[16] R. H. Gomer and R. A. Firtel. Cell-autonomous determination of celltype choice in dictyostelium development by cell-cycle phase. Science, 237(4816):758-762, August 1987.

[17] J. C. Coates and A. J. Harwood. Cell-cell adhesion and signal transduction during dictyostelium development. J. Cell Sci., 114(24):4349-4358, December 2001.

[18] D. A. Brock, T. E. Douglas, D. C. Queller, and J. E. Strassmann. Primitive agriculture in a social amoeba. Nature, 469(7330):393-+, January 2011.

[19] D. A. Cotter and K. B. Raper. Properties of germinating spores of dictyostelium discoideum. J. Bacteriol., 96(5):1680-\&, 1968.

[20] D. A. Cotter and K. B. Raper. Factors affecting rate of heat-induced spore germination in dictyostelium discoideum. J. Bacteriol., 96(1):86-\&, 1968.

[21] M. J. Carnell and R. H. Insall. Actin on disease - studying the pathobiology of cell motility using dictyostelium discoideum. Sem. Cell Dev. Biol., 22(1):82-88, February 2011.

[22] P. Cosson and T. Soldati. Eat, kill or die: when amoeba meets bacteria. Curr. Opin. Microbiol., 11(3):271-276, June 2008.

[23] J. King and R. H. Insall. Parasexual genetics of dictyostelium gene disruptions: identification of a ras pathway using diploids. Bmc Genet., 4:12, July 2003.

[24] A. Kuspa and W. F. Loomis. Tagging developmental genes in dictyostelium by restriction enzyme-mediated integration of plasmid dna. Proc. Natl. Acad. Sci. U. S. A., 89(18):8803-8807, September 1992.

[25] H. Martens, J. Novotny, J. Oberstrass, T. L. Steck, P. Postlethwait, and W. Nellen. Rnai in dictyostelium: The role of rna-directed rna polymerases and double-stranded rnase. Mol. Biol. Cell, 13(2):445-453, February 2002. 
[26] L. Eichinger, J. A. Pachebat, G. Glockner, M. A. Rajandream, R. Sucgang, M. Berriman, J. Song, R. Olsen, K. Szafranski, Q. Xu, B. Tunggal, S. Kummerfeld, M. Madera, B. A. Konfortov, F. Rivero, A. T. Bankier, R. Lehmann, N. Hamlin, R. Davies, P. Gaudet, P. Fey, K. Pilcher, G. Chen, D. Saunders, E. Sodergren, P. Davis, A. Kerhornou, X. Nie, N. Hall, C. Anjard, L. Hemphill, N. Bason, P. Farbrother, B. Desany, E. Just, T. Morio, R. Rost, C. Churcher, J. Cooper, S. Haydock, N. van Driessche, A. Cronin, I. Goodhead, D. Muzny, T. Mourier, A. Pain, M. Lu, D. Harper, R. Lindsay, H. Hauser, K. James, M. Quiles, M. M. Babu, T. Saito, C. Buchrieser, A. Wardroper, M. Felder, M. Thangavelu, D. Johnson, A. Knights, H. Loulseged, K. Mungall, K. Oliver, C. Price, M. A. Quail, H. Urushihara, J. Hernandez, E. Rabbinowitsch, D. Steffen, M. Sanders, J. Ma, Y. Kohara, S. Sharp, M. Simmonds, S. Spiegler, A. Tivey, S. Sugano, B. White, D. Walker, J. Woodward, T. Winckler, Y. Tanaka, G. Shaulsky, M. Schleicher, G. Weinstock, A. Rosenthal, E. C. Cox, R. L. Chisholm, R. Gibbs, W. F. Loomis, M. Platzer, R. R. Kay, J. Williams, P. H. Dear, A. A. Noegel, B. Barrell, and A. Kuspa. The genome of the social amoeba dictyostelium discoideum. Nature, 435(7038):43-57, May 2005.

[27] T. D. Pollard and J. A. Cooper. Actin, a central player in cell shape and movement. Science, 326(5957):1208-1212, November 2009.

[28] R. Dominguez and K. C. Holmes. Actin structure and function. Annu. Rev. Biophys., 40:169-186, 2011.

[29] T. D. Pollard and G. G. Borisy. Cellular motility driven by assembly and disassembly of actin filaments (vol 112, pg 453, 2002). Cell, 113(4):549-549, May 2003.

[30] T. D. Pollard. Regulation of actin filament assembly by arp $2 / 3$ complex and formins. Annu. Rev. Biophys. Biomol. Struct., 36:451-477, 2007.

[31] R. C. Robinson, K. Turbedsky, D. A. Kaiser, J. B. Marchand, H. N. Higgs, S. Choe, and T. D. Pollard. Crystal structure of arp2/3 complex. Science, 294(5547):1679-1684, November 2001.

[32] K. J. Amann and T. D. Pollard. The arp $2 / 3$ complex nucleates actin filament branches from the sides of pre-existing filaments. Nat. Cell Biol., 3(3):306-310, March 2001.

[33] T. M. Svitkina and G. G. Borisy. Arp2/3 complex and actin depolymerizing factor cofilin in dendritic organization and treadmilling of actin filament array in lamellipodia. J. Cell Biol., 145(5):1009-1026, May 1999.

[34] T. D. Pollard, L. Blanchoin, and R. D. Mullins. Molecular mechanisms controlling actin filament dynamics in nonmuscle cells. Annu. Rev. Biophys. Biomol. Struct., 29:545-576, 2000. 
[35] H. Aizawa, Y. Fukui, and I. Yahara. Live dynamics of dictyostelium cofilin suggests a role in remodeling actin latticework into bundles. J. Cell Sci., 110:2333-2344, October 1997.

[36] H. Aizawa, K. Sutoh, S. Tsubuki, S. Kawashima, A. Ishii, and I. Yahara. Identification, characterization, and intracellular-distribution of cofilin in dictyostelium-discoideum. J. Biol. Chem., 270(18):10923-10932, May 1995.

[37] H. Aizawa, M. Katadae, M. Maruya, M. Sameshima, K. Murakami-Murofushi, and I. Yahara. Hyperosmotic stress-induced reorganization of actin bundles in dictyostelium cells over-expressing cofilin. Genes Cells, 4(6):311-324, June 1999.

[38] K. Okada, T. Obinata, and H. Abe. Xaip1: a xenopus homologue of yeast actin interacting protein 1 (aip1), which induces disassembly of actin filaments cooperatively with adf cofilin family proteins. J. Cell Sci., 112(10):1553-1565, May 1999.

[39] S. Ono. Regulation of actin filament dynamics by actin depolymerizing factor/cofilin and actin-interacting protein 1: New blades for twisted filament. Biochemistry (Mosc.), 42(46):13363-13370, November 2003.

[40] S. Ono, K. Mohri, and K. Ono. Microscopic evidence that actin-interacting protein 1 actively disassembles actin-depolymerizing factor/cofilin-bound actin filaments. J. Biol. Chem., 279(14):14207-14212, April 2004.

[41] K. Okada, L. Blanchoin, H. Abe, H. Chen, T. D. Pollard, and J. R. Bamburg. Xenopus actin-interacting protein 1 (xaip1) enhances cofilin fragmentation of filaments by capping filament ends. J. Biol. Chem., 277(45):43011-43016, November 2002.

[42] K. Okada, H. Ravi, E. M. Smith, and B. L. Goode. Aip1 and cofilin promote rapid turnover of yeast actin patches and cables: A coordinated mechanism for severing and capping filaments. Mol. Biol. Cell, 17(7):2855-2868, July 2006.

[43] A. Rodal, J. Tetrault, D. Amberg, and D. Drubin. Aip1p functionally interacts with cofilin in vivo and in vitro. Mol. Biol. Cell, 9:15A-15A, November 1998.

[44] A. A. Rodal, J. W. Tetreault, P. Lappalainen, D. G. Drubin, and D. C. Amberg. Aip1p interacts with cofilin to disassemble actin filaments. J. Cell Biol., 145(6):1251-1264, June 1999.

[45] A. Konzok, I. Weber, E. Simmeth, U. Hacker, M. Maniak, and A. MullerTaubenberger. Daip1, a dictyostelium homologue of the yeast actin-interacting protein 1, is involved in endocytosis, cytokinesis, and motility. J. Cell Biol., 146(2):453-464, July 1999. 
[46] E. L. Dehostos, B. Bradtke, F. Lottspeich, R. Guggenheim, and G. Gerisch. Coronin, an actin binding-protein of dictyostelium-discoideum localized to cell-surface projections, has sequence similarities to g-protein beta-subunits. EMBO J., 10(13):4097-4104, December 1991.

[47] B. L. Goode, J. J. Wong, A. C. Butty, M. Peter, A. L. McCormack, J. R. Yates, D. G. Drubin, and G. Barnes. Coronin promotes the rapid assembly and crosslinking of actin filaments and may link the actin and microtubule cytoskeletons in yeast. J. Cell Biol., 144(1):83-98, January 1999.

[48] L. Cai, Al. M. Makhov, and J. E. Bear. F-actin binding is essential for coronin 1b function in vivo. J. Cell Sci., 120(10):1779-1790, May 2007.

[49] Z. Spoerl, M. Stumpf, A. A. Noegel, and A. Hasse. Oligomerization, f-actin interaction, and membrane association of the ubiquitous mammalian coronin 3 are mediated by its carboxyl terminus. J. Biol. Chem., 277(50):48858-48867, December 2002.

[50] T. Oku, S. Itoh, R. Ishii, K. Suzuki, W. M. Nauseef, S. Toyoshima, and T. Tsuji. Homotypic dimerization of the actin-binding protein p57/coronin-1 mediated by a leucine zipper motif in the c-terminal region. Biochem. J., 387:325-331, April 2005.

[51] J. Gatfield, I. Albrecht, B. Zanolari, M. O. Steinmetz, and J. Pieters. Association of the leukocyte plasma membrane with the actin cytoskeleton through coiled coil-mediated trimeric coronin 1 molecules. Mol. Biol. Cell, 16(6):2786-2798, June 2005.

[52] M. Gandhi, V. Achard, L. Blanchoin, and B. L. Goode. Coronin switches roles in actin disassembly depending on the nucleotide state of actin. Mol. Cell, 34(3):364-374, May 2009.

[53] C. L. Humphries, H. I. Balcer, J. L. D’Agostino, B. Winsor, D. G. Drubin, G. Barnes, B. J. Andrews, and B. L. Goode. Direct regulation of arp2/3 complex activity and function by the actin binding protein coronin. J. Cell Biol., 159(6):993-1004, December 2002.

[54] C. Z. Liu, Y. Chen, and S. F. Sui. The identification of a new actin-binding region in p57. Cell Res., 16(1):106-112, January 2006.

[55] M. Gandhi and B. L. Goode. Coronin: The double-edged sword of actin dynamics. Subcell. Biochem., 48:72-87, 2008.

[56] N. Foeger, L. Rangell, D. M. Danilenko, and A. C. Chan. Requirement for coronin 1 in t lymphocyte trafficking and cellular homeostasis. Science, 313(5788):839-842, August 2006. 
[57] L. Cai, N. Holoweckyj, M. D. Schaller, and J. E. Bear. Phosphorylation of coronin $1 \mathrm{~b}$ by protein kinase c regulates interaction with arp2/3 and cell motility. J. Biol. Chem., 280(36):31913-31923, September 2005.

[58] E. L. Dehostos, C. Rehfuess, B. Bradtke, D. R. Waddell, R. Albrecht, J. Murphy, and G. Gerisch. Dictyostelium mutants lacking the cytoskeletal protein coronin are defective in cytokinesis and cell motility. J. Cell Biol., 120(1):163-173, January 1993.

[59] A. A. Noegel and M. Schleicher. The actin cytoskleleton of dictyostelium: a story told by mutants. J. Cell Sci., 113(5):759-766, March 2000.

[60] M. A. de la Roche and G. P. Cote. Regulation of dictyostelium myosin i and ii. Biochim. Biophys. Acta, 1525(3):245-261, March 2001.

[61] M. Vicente-Manzanares, X. Ma, R. S. Adelstein, and A. R. Horwitz. Non-muscle myosin ii takes centre stage in cell adhesion and migration. Nat. Rev. Mol. Cell Biol., 10(11):778-790, November 2009.

[62] T. Q. P. Uyeda, P. D. Abramson, and J. A. Spudich. The neck region of the myosin motor domain acts as a lever arm to generate movement. Proc. Natl. Acad. Sci. U. S. A., 93(9):4459-4464, April 1996.

[63] C. T. Murphy, R. S. Rock, and J. A. Spudich. A myosin ii mutation uncouples atpase activity from motility and shortens step size. Nat. Cell Biol., 3(3):311315, March 2001.

[64] A. D. Mclachlan and J. Karn. Periodic charge-distributions in the myosin rod amino-acid-sequence match cross-bridge spacings in muscle. Nature, 299(5880):226-231, 1982.

[65] M. A. De la Roche, J. L. Smith, V. Betapudi, T. T. Egelhoff, and G. P. Cote. Signaling pathways regulating dictyostelium myosin ii. J. Muscle Res. Cell Motil., 23(7-8):703-718, October 2002.

[66] S. Yumura and T. Kitanishiyumura. Immunoelectron microscopic studies of the ultrastructure of myosin-filaments in dictyostelium-discoideum. Cell Struct. Funct., 15(6):343-354, December 1990.

[67] C. Pasternak, J. A. Spudich, and E. L. Elson. Capping of surface-receptors and concomitant cortical tension are generated by conventional myosin. Nature, 341(6242):549-551, October 1989.

[68] W. C. Liang, H. M. Warrick, and J. A. Spudich. A structural model for phosphorylation control of dictyostelium myosin ii thick filament assembly. J. Cell Biol., 147(5):1039-1047, November 1999.

[69] L. Bosgraaf and P. J. M. van Haastert. The regulation of myosin ii in dictyostelium. Eur. J. Cell Biol., 85(9-10):969-979, September 2006. 
[70] Y. E. Goldman. Wag the tail: Structural dynamics of actomyosin. Cell, 93(1):14, April 1998.

[71] R. J. Barsotti, J. A. Dantzig, and Y. E. Goldman. Myosin isoforms show different strokes for different blokes. Nat. Struct. Biol., 3(9):737-739, September 1996.

[72] G. Laevsky and D. A. Knecht. Cross-linking of actin filaments by myosin ii is a major contributor to cortical integrity and cell motility in restrictive environments. J. Cell Sci., 116(18):3761-3770, September 2003.

[73] T. T. Egelhoff, D. J. Manstein, and J. A. Spudich. Complementation of myosin null mutants in dictyostelium-discoideum by direct functional selection. Dev. Biol., 137(2):359-367, February 1990.

[74] L. A. Cameron, P. A. Giardini, F. S. Soo, and J. A. Theriot. Secrets of actinbased motility revealed by a bacterial pathogen. Nat. Rev. Mol. Cell Biol., 1(2):110-119, November 2000.

[75] P. Cossart and A. Toledo-Arana. Listeria monocytogenes, a unique model in infection biology: an overview. Microbes Infect., 10(9):1041-1050, July 2008.

[76] M. Kaksonen, C. P. Toret, and D. G. Drubin. Harnessing actin dynamics for clathrin-mediated endocytosis. Nat. Rev. Mol. Cell Biol., 7(6):404-414, June 2006.

[77] V. Laurent, T. P. Loisel, B. Harbeck, A. Wehman, L. Grobe, B. M. Jockusch, J. Wehland, F. B. Gertler, and M. F. Carlier. Role of proteins of the ena/vasp family in actin-based motility of listeria monocytogenes. J. Cell Biol., 144(6):1245-1258, March 1999.

[78] S. M. Rafelski and J. A. Theriot. Mechanism of polarization of listeria monocytogenes surface protein acta. Mol. Microbiol., 59(4):1262-1279, February 2006.

[79] G. Gerisch. Self-organizing actin waves that simulate phagocytic cup structures. PMC Biophys, 3(1):7-7, March 2010.

[80] T. Bretschneider, S. Diez, K. Anderson, J. Heuser, M. Clarke, A. MullerTaubenberger, J. Kohler, and G. Gerisch. Dynamic actin patterns and arp2/3 assembly at the substrate-attached surface of motile cells. Curr. Biol., 14(1):110, January 2004.

[81] Y. Asano, A. Nagasaki, and T. Q. P. Uyeda. Correlated waves of actin filaments and pip(3) in dictyostelium cells. Cell Motil. Cytoskeleton, 65(12):923-934, December 2008.

[82] G. Gerisch, T. Bretschneider, A. Muller-Taubenberger, E. Simmeth, M. Ecke, S. Diez, and K. Anderson. Mobile actin clusters and traveling waves in 
cells recovering from actin depolymerization. Biophys. J., 87(5):3493-3503, November 2004.

[83] G. Gerisch, M. Ecke, D. Wischnewski, and B. Schroth-Diez. Different modes of state transitions determine pattern in the phosphatidylinositide-actin system. BMC Cell Biol., 12:42, October 2011.

[84] A. Goldbeter, C. Gerard, D. Gonze, J. C. Leloup, and G. Dupont. Systems biology of cellular rhythms. FEBS Lett., 586(18):2955-2965, August 2012.

[85] K. Kruse and F. Julicher. Oscillations in cell biology. Curr. Opin. Cell Biol., 17(1):20-26, February 2005.

[86] A. Goldbeter, C. Gerard, and J. C. Leloup. Circadian rhythms and systems biology. Med. Sci., 26(1):49-56, January 2010.

[87] C. L. Partch, C. B. Green, and J. S. Takahashi. Molecular architecture of the mammalian circadian clock. Trends Cell Biol., 24(2):90-99, February 2014.

[88] C. Dibner, U. Schibler, and U. Albrecht. The mammalian circadian timing system: Organization and coordination of central and peripheral clocks. Annu. Rev. Physiol., 72:517-549, 2010.

[89] G. Meacci and K. Kruse. Min-oscillations in escherichia coli induced by interactions of membrane-bound proteins. Phys. Biol., 2(2):89-97, June 2005.

[90] M. Loose, K. Kruse, and P. Schwille. Protein self-organization: Lessons from the min system. Annu. Rev. Biophys., 40:315-336, 2011.

[91] C. Shingyoji, H. Higuchi, M. Yoshimura, E. Katayama, and T. Yanagida. Dynein arms are oscillating force generators. Nature, 393(6686):711-714, June 1998.

[92] A. C. Crawford and R. Fettiplace. The mechanical-properties of ciliary bundles of turtle cochlear hair-cells. J. Physiol. (Lond.), 364(JUL):359-\&, 1985.

[93] G. Gerisch, D. Hulser, D. Malchow, and U. Wick. Cell communication by periodic cyclic-amp pulses. Phil. Trans. R. Soc. B, 272(915):181-\&, 1975.

[94] B. M. Shaffer. Secretion of cyclic-amp induced by cyclic-amp in cellular slime-mold dictyostelium-discoideum. Nature, 255(5509):549-552, 1975.

[95] R. P. Futrelle, J. Traut, and W. G. Mckee. Cell behavior in dictyosteliumdiscoideum - pre-aggregation response to localized cyclic-amp pulses. J. Cell Biol., 92(3):807-821, 1982.

[96] E. Schaefer, D. Aue, M. Tarantola, E. Polo, C. Westendorf, N. Oikawa, E. Bodenschatz, B. Geil, and A. Janshoff. Collective behavior of dictyostelium discoideum monitored by impedance analysis. Commun. Integr. Biol., 6(3):e23894-e23894, May 2013. 
[97] D. Malchow, R. Bohme, and U. Gras. On the role of calcium in chemotaxis and oscillations of dictyostelium cells. Biophys. Struct. Mech., 9(2):131-136, 1982.

[98] J. Bumann, B. Wurster, and D. Malchow. Attractant-induced changes and oscillations of the extracellular ca ++ concentration in suspensions of differentiating dictyostelium cells. J. Cell Biol., 98(1):173-178, 1984.

[99] Y. Arai, T. Shibata, S. Matsuoka, M. J. Sato, T. Yanagida, and M. Ueda. Selforganization of the phosphatidylinositol lipids signaling system for random cell migration. Proc. Natl. Acad. Sci. U. S. A., 107(27):12399-12404, July 2010.

[100] T. Shibata, A. Yoshiyuki, M. Satomi, S. J. Masayuki, and U. Masahiro. Selforganization of the phosphatidylinositol lipids signaling system for random cell migration. Biophys. J., 100(3):Biophys Soc, February 2011.

[101] L. Bosgraaf and P. J. M. Van Haastert. Quimp3, an automated pseudopodtracking algorithm. Cell Adh. Migr., 4(1):46-55, January 2010.

[102] P. J. M. Van Haastert. Chemotaxis: insights from the extending pseudopod. $J$. Cell Sci., 123(18):3031-3037, September 2010.

[103] P. J. M. Van Haastert. A stochastic model for chemotaxis based on the ordered extension of pseudopods. Biophys. J., 99(10):3345-3354, November 2010.

[104] A. Kortholt, I. Keizer-Gunnink, R. Kataria, and P. J. M. Van Haastert. Ras activation and symmetry breaking during dictyostelium chemotaxis. $\mathrm{J}$. Cell Sci., 126(19):4502-4513, October 2013.

[105] P. J. M. Van Haastert. How cells use pseudopods for persistent movement and navigation. Sci. Signal., 4(159):pe6, February 2011.

[106] P. J. M. van Haastert and M. Postma. Biased random walk by stochastic fluctuations of chemoattractant-receptor interactions at the lower limit of detection. Biophys. J., 93(5):1787-1796, September 2007.

[107] P. J. M. Van Haastert and L. Bosgraaf. The local cell curvature guides pseudopodia towards chemoattractants. HFSP J, 3(4):282-286, August 2009.

[108] L. Bosgraaf and P. J. M. Van Haastert. Navigation of chemotactic cells by parallel signaling to pseudopod persistence and orientation. Plos One, 4(8):e6842, August 2009.

[109] C. Westendorf, J. Negrete, A. J. Bae, R. Sandmann, E. Bodenschatz, and C. Beta. Actin cytoskeleton of chemotactic amoebae operates close to the onset of oscillations. Proc. Natl. Acad. Sci. U. S. A., 110(10):3853-3858, March 2013. 
[110] C.-H. Huang, M. Tang, C. Shi, P. A. Iglesias, and P. N. Devreotes. An excitable signal integrator couples to an idling cytoskeletal oscillator to drive cell migration. Nat. Cell Biol., 15(11):1307-U108, November 2013.

[111] Nano SU-8 Negative tone photoresist formulations 2-25.

[112] Y. N. Xia and G. M. Whitesides. Soft lithography. Annu. Rev. Mater. Sci., 28:153-184, 1998.

[113] S. J. Clarson and J. A. Semlyen. Siloxane Polymers. Englewood Cliffs, NJ:Prentice Hall, 1993.

[114] R. H. Kessin. Dictyostelium: evolution, cell biology, and the development of multicellularity. Developmental and Cell Biology Series, 38:i-xiv, 2001.

[115] V. Hagen, J. Bendig, S. Frings, T. Eckardt, S. Helm, D. Reuter, and U. B. Kaupp. Highly efficient and ultrafast phototriggers for camp and cgmp by using longwavelength uv/vis-activation. Angew. Chem. Int. Ed., 40(6):1046-+, 2001.

[116] V. Hagen, S. Frings, J. Bendig, D. Lorenz, B. Wiesner, and U. B. Kaupp. Fluorescence spectroscopic quantification of the release of cyclic nucleotides from photocleavable [bis(carboxymethoxy)coumarin-4-yl]methyl esters inside cells. Angew. Chem. Int. Ed., 41(19):3625-3628, 2002.

[117] C. Westendorf. Oscillatory dynamics of the actin Cytoskeleton. PhD thesis, Georg-August-Universität Göttingen, 2013.

[118] C. Beta, D. Wyatt, W.-J. Rappel, and E. Bodenschatz. Flow photolysis for spatiotemporal stimulation of single cells. Anal. Chem., 79(10):3940-3944, May 2007.

[119] D. Malchow, V. Nanjundiah, and G. Gerisch. Ph oscillations in cell-suspensions of dictyostelium-discoideum - their relation to cyclic-amp signals. J. Cell Sci., 30(APR):319-330, 1978.

[120] T. Killich, P. J. Plath, E. C. Hass, W. Xiang, H. Bultmann, L. Rensing, and M. G. Vicker. Cell-movement and shape are nonrandom and determined by intracellular, oscillatory rotating waves in dictyostelium amebas. Biosystems, 33(2):75-87, 1994.

[121] H. C. Ishikawa-Ankerhold. Functional Organization of the Actin System in Dictyostelium. PhD thesis, Technischen Universität München, 2008.

[122] M. Wu, X. Wu, and P. De Camilli. Calcium oscillations-coupled conversion of actin travelling waves to standing oscillations. Proc. Natl. Acad. Sci. U. S. A., 110(4):1339-1344, January 2013. 
[123] J. Bumann, D. Malchow, and B. Wurster. Oscillations of ca ++ concentration during the cell-differentiation of dictyostelium-discoideum - their relation to oscillations in cyclic-amp and other components. Differentiation, 31(2):85-91, 1986.

[124] A. Goldbeter. Oscillations and waves of cyclic amp in dicyostelium: A prototype for spatio-temporal organization and pulsatile intercellular communication. Bull. Math. Biol., 68(5):1095-1109, July 2006.

[125] V. Nanjundiah. Cyclic amp oscillations in dictyostelium discoideum: models and observations. Biophys. Chem., 72(1-2):1-8, May 1998.

[126] J. Negrete. Fluctuations and Oscillatory Instabilities of Intracellular Fiber Networks. PhD thesis, Georg-August-Universität Göttingen, 2014.

[127] R. J. Eddy, J. Han, and J. S. Condeelis. Capping protein terminates but does not initiate chemoattractant-induced actin assembly in dictyostelium. J. Cell Biol., 139(5):1243-1253, December 1997.

[128] D. Yarar, W. To, A. Abo, and M. D. Welch. The wiskott-aldrich syndrome protein directs actin-based motility by stimulating actin nucleation with the arp2/3 complex. Curr. Biol., 9(10):555-558, May 1999.

[129] D. Winter, T. Lechler, and R. Li. Activation of the yeast arp $2 / 3$ complex by bee1p, a wasp-family protein. Curr. Biol., 9(9):501-504, May 1999.

[130] R. Rohatgi, L. Ma, H. Miki, M. Lopez, T. Kirchhausen, T. Takenawa, and M. W. Kirschner. The interaction between n-wasp and the arp2/3 complex links cdc42-dependent signals to actin assembly. Cell, 97(2):221-231, April 1999.

[131] L. M. Machesky, R. D. Mullins, H. N. Higgs, D. A. Kaiser, L. Blanchoin, R. C. May, M. E. Hall, and T. D. Pollard. Scar, a wasp-related protein, activates nucleation of actin filaments by the arp2/3 complex. Proc. Natl. Acad. Sci. U. S. A., 96(7):3739-3744, March 1999.

[132] P. J. M. Van Haastert and L. Bosgraaf. Food searching strategy of amoeboid cells by starvation induced run length extension. Plos One, 4(8):e6814, August 2009.

[133] D. Wessels, D. R. Soll, D. Knecht, W. F. Loomis, A. Delozanne, and J. Spudich. Cell motility and chemotaxis in dictyostelium amebae lacking myosin heavychain. Dev. Biol., 128(1):164-177, July 1988.

[134] J. A. Spudich. In pursuit of myosin function. Cell Regul., 1(1):1-11, November 1989.

[135] D. Wessels and D. R. Soll. Myosin-ii heavy-chain null mutant of dictyostelium exhibits defective intracellular particle movement. J. Cell Biol., 111(3):11371148, September 1990. 
[136] P. J. Heid, D. Wessels, K. J. Daniels, P. Gibson, H. Zhang, E. Voss, and D. R. Soll. The role of myosin heavy chain phosphorylation in dictyostelium motility, chemotaxis and f-actin localization. J. Cell Sci., 117(20):4819-4835, September 2004.

[137] C. Westendorf, A. J. Bae, C. Erlenkamper, E. Galland, C. Franck, E. Bodenschatz, and C. Beta. Live cell flattening - traditional and novel approaches. PMC Biophys, 3(1):9-9, April 2010.

[138] I. Sadler, A. W. Crawford, J. W. Michelsen, and M. C. Beckerle. Zyxin and ccrp - 2 interactive lim domain proteins associated with the cytoskeleton. J. Cell Biol., 119(6):1573-1587, December 1992.

[139] I. Kovacic and M. J. Brennan, editors. The Duffing Equation: Nonlinear Oscillators and their Behaviour. John Wiley \& Sons, Ltd., 2011.

[140] C. J. Wang, A. Bergmann, B. Lin, K. Kim, and A. Levchenko. Diverse sensitivity thresholds in dynamic signaling responses by social amoebae. Sci. Signal., 5(213):ra17, February 2012.

[141] A. Samadani, J. Mettetal, and A. van Oudenaarden. Cellular asymmetry and individuality in directional sensing. Proc. Natl. Acad. Sci. U. S. A., 103(31):1154911554, August 2006.

[142] P. Lappalainen, E. V. Fedorov, A. A. Fedorov, S. C. Almo, and D. G. Drubin. Essential functions and actin-binding surfaces of yeast cofilin revealed by systematic mutagenesis. EMBO J., 16(18):5520-5530, September 1997.

[143] J. D. Murray. Mathematical Biology. Springer, 2004.

[144] E. Shelden and D. A. Knecht. Dictyostelium cell shape generation requires myosin ii. Cell Motil. Cytoskeleton, 35(1):59-67, 1996.

[145] P. A. Clow and J. G. McNally. In vivo observations of myosin ii dynamics support a role in rear retraction. Mol. Biol. Cell, 10(5):1309-1323, May 1999.

[146] E. Shelden and D. A. Knecht. Mutants lacking myosin-ii cannot resist forces generated during multicellular morphogenesis. J. Cell Sci., 108:1105-1115, March 1995.

[147] M. G. Lemieux, D. Janzen, R. Hwang, J. Roldan, and D. A. Jarchum, I.and Knecht. Visualization of the actin cytoskeleton: Different f-actin-binding probes tell different stories. Cytoskeleton, 71(3):157-169, March 2014.

[148] S. Yumura. How does myosin ii localize within a dictyostelium cell? J. Plant Res., 110(1100):501-510, December 1997. 
[149] R. K. Mahajan, K. T. Vaughan, J. A. Johns, and J. D. Pardee. Actin-filaments mediate dictyostelium myosin assembly invitro. Proc. Natl. Acad. Sci. U. S. A., 86(16):6161-6165, August 1989.

[150] S. Levi, M. V. Polyakov, and T. T. Egelhoff. Myosin ii dynamics in dictyostelium: Determinants for filament assembly and translocation to the cell cortex during chemoattractant responses. Cell Motil. Cytoskeleton, 53(3):177-188, November 2002 .

[151] V. T. Nachmias, Y. Fukui, and J. A. Spudich. Chemoattractant-elicited translocation of myosin in motile dictyostelium. Cell Motil. Cytoskeleton, 13(3):158-169, 1989.

[152] A. Ryter, C. Klein, and P. Brachet. Dictyostelium-discoideum surface changes elicited by high-concentrations of camp. Exp. Cell Res., 119(2):373-380, 1979.

[153] J. Condeelis, A. Hall, A. Bresnick, V. Warren, R. Hock, H. Bennett, and S. Ogihara. Actin polymerization and pseudopod extension during ameboid chemotaxis. Cell Motil. Cytoskeleton, 10(1-2):77-90, 1988.

[154] L. Haviv, D. Gillo, Fr. Backouche, and A. Bernheim-Groswasser. A cytoskeletal demolition worker: Myosin ii acts as an actin depolymerization agent. J. Mol. Biol., 375(2):325-330, January 2008.

[155] P. Latimer. Light-scattering vs microscopy for measuring average cell-size and shape. Biophys. J., 27(1):117-126, 1979.

[156] D. A. Knecht and W. F. Loomis. Antisense rna inactivation of myosin heavychain gene-expression in dictyostelium-discoideum. Science, 236(4805):10811086, May 1987.

[157] G. Gerisch and I. Weber. Cytokinesis without myosin ii. Curr. Opin. Cell Biol., 12(1):126-132, February 2000.

[158] A. De Lozanne and J. A. Spudich. Disruption of the dictyostelium myosin heavy-chain gene by homologous recombination. Science, 236(4805):10861091, May 1987.

[159] D. J. Manstein, M. A. Titus, A. De Lozanne, and J. A. Spudich. Gene replacement in dictyostelium - generation of myosin null mutants. EMBO J., 8(3):923-932, March 1989.

[160] J. H. Zang, G. Cavet, J. H. Sabry, P. Wagner, S. L. Moores, and J. A. Spudich. On the role of myosin-ii in cytokinesis: Division of dictyostelium cells under adhesive and nonadhesive conditions. Mol. Biol. Cell, 8(12):2617-2629, December 1997.

[161] T. Q. P. Uyeda, A. Nagasaki, and S. Yumura. Multiple parallelisms in animal cytokinesis. Int. Rev. Cytol., 240:377-+, 2004. 
[162] R. Neujahr, C. Heizer, and G. Gerisch. Myosin ii-independent processes in mitotic cells of dictyostelium discoideum: Redistribution of the nuclei, rearrangement of the actin system and formation of the cleavage furrow. $J$. Cell Sci., 110:123-137, January 1997.

[163] I. Weber, R. Neujahr, A. P. Du, J. Kohler, J. Faix, and G. Gerisch. Two-step positioning of a cleavage furrow by cortexillin and myosin ii. Curr. Biol., 10(9):501-506, May 2000.

[164] C. Janetopoulos and R. A. Firtel. Directional sensing during chemotaxis. FEBS Lett., 582(14):2075-2085, June 2008.

[165] M. Etzrodt, H. C. F. Ishikawa, J. Dalous, A. Mueller-Taubenberger, T. Bretschneider, and G. Gerisch. Time-resolved responses to chemoattractant, characteristic of the front and tall of dictyostelium cells. FEBS Lett., 580(28-29):6707-6713, December 2006.

[166] M. Postma, J. Roelofs, J. Goedhart, H. M. Loovers, A. J. W. G. Visser, and P. J. M. Van Haastert. Sensitization of dictyostelium chemotaxis by phosphoinositide-3kinase-mediated self-organizing signalling patches. J. Cell Sci., 117(14):29252935, June 2004.

[167] M. Postma, J. Roelofs, J. Goedhart, T. W. J. Gadella, A. J. W. G. Visser, and P. J. M. Van Haastert. Uniform camp stimulation of dictyostelium cells induces localized patches of signal transduction and pseudopodia. Mol. Biol. Cell, 14(12):5019-5027, December 2003. 


\section{Acknowledgments}

The thesis cannot be finished without the support from many people. First, I would like to thank my advisor Prof. Dr. Eberhard Bodenschatz for giving me the chance to work here. His support and advices make the work better. I would like to thank my second advisor Prof. Dr. Carsten Beta for all the support and discussions to improve the work. Many thanks Prof. Dr. Andreas Janshoff and Prof. Dr. Jörg Enderlein for being my thesis committee members to help me finish on the right track. I would also like to thank Prof. Dr. Alain Pumir for his discussions and valuable opinions. I would like to thank Prof. Dr. Thomas Burg, Prof. Dr. Gregor Eichele and Prof. Dr. Jörg Großhans for being the member of the examination board of my thesis defense.

I really appreciate all the great help Katharina, Maren, Marion and Tina in taking care of numerous cells. I am grateful to have nice colleagues. It's really enjoyable to work together with Alexei, Azam, Brian, Christoph, Christian, Isabella, Jose, Kaumudi, Laura, Marco, Noriko and Vladimir. Thanks to Fabio, Garrett, Jennifer, Holly, Michael, Prasanth and Simon for being my roommates. It's very nice to have their accompany and all the helps. Especially thanks to Azam, Isabella, Kaumudi and Laura for taking care of me like my sisters. Jenny is always super helpful. Thanks to Angela for solving all the administration matters and making my life in Germany much easier. Thanks to Andreas Kopp, Andreas Renner, Artur, Dennis, Florian, Gerhard, Haitao, Holger, Sabrina and Xiaozhou for their helps in the past years. Thanks to Antje, Frauke and Tina (PBCS office) to help me cope with administrative matters from the school.

Finally, thanks to Chao-Chen for all the support in life as well as in work. Life would be more difficult and boring without him. Thanks to my family. Their support helps me get through the hard times. Thanks God, I finished! 


\title{
Curriculum Vitae
}

\section{Academic Background}

\author{
2011/10-present \\ $\mathrm{PhD}$ in Physics \\ Max Planck Institute for Dynamics and Self-Organization \\ Georg-August-Universität Göttingen \\ Thesis topic: \\ Oscillatory instabilities of intracellular fiber networks \\ Advisor: Prof. Dr. Eberhard Bodenschatz \\ 2010/08-2011/09 Research assistant \\ National Taiwan University, Department of Chemistry \\ 2008/09-2010/07 M.S., Department of Chemistry \\ National Taiwan University \\ Thesis topic: \\ Investigating RecA filament formation of Escherichia coli and \\ Deinococcus radiodurans along duplex DNA \\ Advisor: Prof. Dr. Hung-Wen Li \\ - Master thesis awarded as top 5 in the department. \\ - Dissertation award: Annual meeting of the Chinese chemical \\ society. \\ 2004/09-2008/06 B.S., Department of Chemistry \\ National Taiwan University
}

\section{Publications}

1. Hsu, H.-F., Ngo, K. N., Chitteni-Pattu, S., Cox, M. M. \& Li, H.-W., "Investigating Deinococcus radiodurans RecA protein filament formation by real-time singlemolecule approaches" Biochemistry 2011, 50, 8270-8280

2. Piechura, J. R., Tseng, T.-L., Hsu, H.-F., Byrne, R.T., Battista, J. R., Li, H.-W. \& Cox, M. M.,"Directed Evolution of Extreme Resistance to Ionizing Radiation: How RecA Protein Adaptation Contributes." DNA Repair 2015, 26, 30-43.

3. Hsu, H.-F., Westendorf, C., Tarantola, M., Zykov, V., Bodenschatz, E., Beta, C., "Cell-to-cell variability in the actin polymerization rate controls the intrinsic frequency of a nonlinear cytoskeletal oscillator." (in preparation)

4. Hsu, H.-F., Tarantola, M., Beta, C., Bodenschatz, E., "Myosin II sets the optimal response time scale of chemotatic amoeba." (in preparation) 